\title{
Biholomorphic equivalence to totally nondegenerate model CR manifolds
}

\author{
Masoud Sabzevari ${ }^{1,2}$ (D)
}

Received: 11 April 2018 / Accepted: 24 November 2018 / Published online: 1 December 2018 (c) Fondazione Annali di Matematica Pura ed Applicata and Springer-Verlag GmbH Germany, part of Springer Nature 2018

\begin{abstract}
Applying Élie Cartan's classical method, we show that the biholomorphic equivalence problem to a totally nondegenerate Beloshapka's model of CR dimension one and codimension $k>1$, whence of real dimension $2+k$, is reducible to some absolute parallelism, namely to an $\{e\}$-structure on a certain prolonged manifold of real dimension either $3+k$ or $4+k$. The proof relies upon a careful weight analysis on the structure equations associated with the mentioned problem of equivalence. As one of the applications of the achieved results, we also reconfirm in CR dimension one Beloshapka's maximum conjecture on the holomorphic rigidity of his models in certain lengths equal or greater than three.
\end{abstract}

Keywords Equivalence problem · Totally nondegenerate manifolds · Maximum conjecture

Mathematics Subject Classification $32 \mathrm{~V} 40 \cdot 58 \mathrm{~A} 15 \cdot 57 \mathrm{~S} 25$

\section{Contents}

1 Introduction . . . . . . . . . . . . . . . . . . . . . . . . 1122

1.1 Existence of totally nondegenerate CR manifolds in arbitrary codimensions . . . . . . . . . 1123

2 Beloshapka's models . . . . . . . . . . . . . . . . . . . . . . . . . . 1130

2.1 Constructing the defining equations . . . . . . . . . . . . . . . . . . . . . . 1131

3 Constructing associated structure equations . . . . . . . . . . . . . . . . . . . 1133

3.1 Associated initial frames for the complexified tangent bundles . . . . . . . . . . . . . . 1133

3.1 .1 Notations . . . . . . . . . . . . . . . . . . . . . . . . . . . . . 1134

3.2 The associated initial coframes and their Darboux-Cartan structures . . . . . . . . . . . . 1136

3.3 Ambiguity matrix . . . . . . . . . . . . . . . . . . . . . . . . . . . . 1137

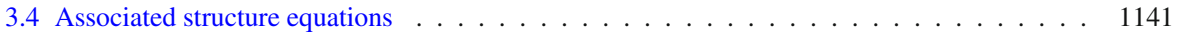

3.5 Torsion coefficients . . . . . . . . . . . . . . . . . . . . . . . . . . . . 1142

4 Weight analysis on the structure equations f . . . . . . . . . . . . . . . . . . . . . . . 1143

5 Picking up an appropriate weighted homogeneous subsystem . . . . . . . . . . . . . . . . . . 1146

5.1 Picking up an appropriate subsystem . . . . . . . . . . . . . . . . . . . . . . . . . . . . 1147

Masoud Sabzevari

sabzevari@math.iut.ac.ir

1 Department of Mathematics, Shahrekord University, Shahrekord 88186-34141, Iran

2 School of Mathematics, Institute for Research in Fundamental Sciences (IPM), Tehran 19395-5746, Iran 
5.1.1 First part: structure equations of the weights $-\ell=-1, \ldots,-(\rho-1) \ldots \ldots 1147$

5.1 .2 Second part: structure equations of the weight $-\rho \ldots \ldots . \ldots \ldots$. . . . . . . 1150

5.2 Solving the picked up subsystem . . . . . . . . . . . . . . . . . . . . . 1151

5.3 Normalization of $a_{1} \ldots \ldots \ldots \ldots \ldots \ldots \ldots$

5.4 Prolongation . . . . . . . . . . . . . . . . . . . . . . . . 1156

6 Proof of Beloshapka's maximum conjecture in CR dimension one . . . . . . . . . . . . . . . . . 1158

Appendix A: An illustrative example in length four . . . . . . . . . . . . . . . . . . . . . 1159

References . . . . . . . . . . . . . . . . . . . . . . . . 1162

\section{Introduction}

The notion of totally nondegenerate CR manifolds has a close connection with the theory of free Lie algebras. In order to explain this connection in CR dimension one, let $h_{1}$ and $h_{2}$ be two linearly independent elements of a certain vector space over the field $\mathbb{C}$. By definition $[20,22,26]$, the rank two complex free Lie algebra $\mathscr{F}$ is the smallest non-commutative and non-associative $\mathbb{C}$-algebra having $h_{1}$ and $h_{2}$ as its elements, with bilinear multiplication $\left(h, h^{\prime}\right) \mapsto\left[h, h^{\prime}\right] \in \mathscr{F}$, satisfying the skew-symmetry and Jacobi-like identity:

$$
\begin{aligned}
& 0=\left[h, h^{\prime}\right]+\left[h^{\prime}, h\right], \\
& 0=\left[h,\left[h^{\prime}, h^{\prime \prime}\right]\right]+\left[h^{\prime \prime},\left[h, h^{\prime}\right]\right]+\left[h^{\prime},\left[h^{\prime \prime}, h\right]\right],
\end{aligned}
$$

for arbitrary elements $h, h^{\prime} h^{\prime \prime} \in \mathscr{F}$. Such an algebra $\mathscr{F}$ is unique up to isomorphism. Importantly, no linear relation exists between iterated multiplications, i.e., between iterated Lie brackets, except those generated only by skew-symmetry and Jacobi identity: this is the freeness of the algebra.

Then, the elements of $\mathscr{F}$, designated as words, can be rewritten as iterated Lie brackets between the letters $h_{1}$ and $h_{2}$; for instance:

$$
\left[\left[h_{1}, h_{2}\right], h_{1}\right], \quad\left[h_{1},\left[h_{1},\left[h_{2}, h_{1}\right]\right]\right], \quad\left[\left[h_{2}, h_{1}\right],\left[h_{1},\left[h_{2}, h_{1}\right]\right]\right] .
$$

We define the length of each word to be the number of $h_{1}, h_{2}$ elements in it. Define $\mathscr{F}_{1}$ to be the $\mathbb{C}$-vector space generated by $h_{1}, h_{2}$ and for $\ell \geqslant 2$, let $\mathscr{F} \ell$ be the $\mathbb{C}$-vector space generated by all words of the lengths $\leqslant \ell$. Then clearly $\mathscr{F}=\bigcup_{\ell \geqslant 1} \mathscr{F} \ell$ and we have the following filtration:

$$
\mathscr{F}_{1} \subset \mathscr{F}_{2} \subset \mathscr{F}_{3} \subset \cdots
$$

on $\mathscr{F}$. Also let us denote by $\mathrm{n}_{\ell}$, dimension of the complex vector space $\mathscr{F} \ell$ which can be computed by means of the recursive relation, introduced in [20, Theorem 2.6]. By an induction based on the Jacobi identity, it follows that each length $\ell$ word can be expressed as a linear combination of some specific words of the form:

$$
\left[h_{i_{1}},\left[h_{i_{2}},\left[\ldots\left[h_{i_{\ell-1}}, h_{i_{\ell}}\right] \ldots\right]\right],\right.
$$

which are called simple words of the length $\ell$. Hence, for each $\ell>1$ we have $\mathscr{F} \ell:=$ $\mathscr{F}_{\ell-1}+\left[\mathscr{F}_{1}, \mathscr{F}_{\ell-1}\right]$. The collection of all simple words generates $\mathscr{F}$ as a vector space over $\mathbb{C}$, though actually it is not a basis. ${ }^{1}$

Let $\mathfrak{F}_{\ell}:=\mathscr{F}_{\ell} / \mathscr{F}_{\ell-1}$ be the $\mathbb{C}$-vector space generated by all (simple) words of length $\ell$. Then, $\mathfrak{F}_{\ell}$ is of dimension $\mathrm{m}_{\ell}:=\mathrm{n}_{\ell}-\mathrm{n}_{\ell-1}$ and $\left[\mathfrak{F}_{\ell_{1}}, \mathfrak{F}_{\ell_{2}}\right] \subset \mathfrak{F}_{\ell_{1}+\ell_{2}}$ for each $\ell_{1}, \ell_{2} \geqslant 1$.

1 One basis for the free Lie algebra $\mathscr{F}$ is the so-called Hall-Witt basis (see [20, Definition 2.5 and Theorem 2.6]). 
Consequently, our infinite dimensional free algebra $\mathscr{F}$ is graded of the form:

$$
\mathfrak{F}_{1} \oplus \mathfrak{F}_{2} \oplus \mathfrak{F}_{3} \oplus \ldots
$$

Now, let us turn our attention to the subject of totally nondegenerate CR manifolds in $\mathrm{CR}$ dimension one. Let $M \subset \mathbb{C}^{1+k}$ be a real analytic generic submanifold of codimension $k$ and hence of real dimension $2+k$. As is known [1,8,20,21], the holomorphic subbundle $T^{1,0} M$ of its complexified bundle $\mathbb{C} \otimes T M$ can be generated by a single holomorphic vector field $\mathscr{L}$. Let $D_{1}:=T^{1,0} M+T^{0,1} M$, where $T^{0,1} M:=\overline{T^{1,0} M}$ and define successively $D_{j}=D_{j-1}+\left[D_{1}, D_{j-1}\right]$ for $j>1$. As is customary in the Lie-Cartan theory, we assume strong uniformity, that is: for each $j \geqslant 1$, the dimension of $D_{j}$ is fully constant on the points of $M$ if it is thought of as being local. So, all $D_{j}$ s are subbundles of $\mathbb{C} \otimes T M$. It is also natural to assume that $M$ is minimal $[1,20,21]$, in the sense that:

$$
D_{i}=\mathbb{C} \otimes T M \quad \text { for all } i \geqslant i^{*} \text { large enough. }
$$

Lastly, as a first step in the study of such differential structures, it is also natural to assume that the ranks of the subbundles $D_{1}, D_{2}, D_{3}, \ldots$ increase as much as possible.

Definition 1.1 An arbitrary (local) real analytic CR generic submanifold $M \subset \mathbb{C}^{1+k}$ of $\mathrm{CR}$ dimension one and codimension $k$ is called totally nondegenerate-or completely nondegenerate or maximally minimal (cf. [21])—whenever $\mathbb{C} \otimes T M$ can be generated by means of the minimum possible number of iterated Lie brackets between the generators $\mathscr{L}$ and $\overline{\mathscr{L}}$ of $D_{1}$, increasing maximally through a filtration:

$$
D_{1} \varsubsetneqq D_{2} \varsubsetneqq \cdots \varsubsetneqq D_{\rho}=\mathbb{C} \otimes T M .
$$

In this case, the length $\rho$ of this filtration is also called by length of $M$.

Set $h_{1}:=\mathscr{L}$ and $h_{2}:=\overline{\mathscr{L}}$. Rephrasing this in the language of free Lie algebras, a real analytic CR generic submanifold $M \subset \mathbb{C}^{1+k}$ of $\mathrm{CR}$ dimension one is totally nondegenerate of the length $\rho$ whenever for each $\ell=1, \ldots, \rho-1$, the vector space $D_{\ell}$ can be identified by its corresponding $\mathscr{F}_{\ell}$. More precisely, whenever the rank of $D_{\ell}$ is maximum, equal to the dimension $\mathrm{n}_{\ell}$ of $\mathscr{F}_{\ell}$ and $D_{\ell}$ behaves precisely as an $\mathrm{n}_{\ell}$-dimensional $\mathbb{C}$-vector space generated by the maximum possible number $\sum_{l \leqslant \ell} \mathrm{m}_{l}$ of (simple) iterated Lie brackets between $\mathscr{L}$ and $\overline{\mathscr{L}}$ of lengths $l \leq \ell$. As is the case with the free Lie algebra $\mathscr{F}$, no linear relation exists between the iterated brackets of $\mathscr{L}$ and $\overline{\mathscr{L}}$ in the lengths $\leq \rho-1$, except those generated by skew-symmetry and Jacobi identity. However, the case of the last bundle $D_{\rho}=\mathbb{C} \otimes T M$ is in part a different matter. Careful inspection of the above definition shows that the length $\rho$ of a $k$-codimensional submanifold $M$ is indeed the smallest integer $\ell$ satisfying:

$$
\operatorname{rank} \mathbb{C} \otimes T M \leq \operatorname{dim} \mathscr{F}_{\ell}
$$

or equivalently $2+k \leqslant \mathrm{n}_{\ell}$. It is of course possible in certain codimensions $k$ that $\rho$ satisfies $2+k \supsetneqq \mathrm{n}_{\rho}$. Such a constraint on the rank of the complexified bundle $\mathbb{C} \otimes T M$ in comparison with the maximal freedom $\mathrm{n}_{\rho}$ may cause an encounter with a length $\rho$ simple iterated bracket $\mathscr{T}_{\rho} \in D_{\rho} / D_{\rho-1}$ which is not independent of the other length $\rho$ simple brackets even modulo skew-symmetry and Jacobi identity.

\subsection{Existence of totally nondegenerate CR manifolds in arbitrary codimensions}

For a CR generic submanifold $M \subset \mathbb{C}^{1+k}$ of codimension $k$, consider the complex tangent space $T^{c} M:=\operatorname{Re}\left(T^{1,0} M\right)$. If $\mathscr{L}:=\mathrm{X}+i \mathrm{Y}$ is the single generator of $T^{1,0} M$, then clearly 
$\mathrm{X}, \mathrm{Y}$ generate $T^{c} M$. As is known $[1,8,20]$ :

$$
\mathbb{C} \otimes T^{c} M=T^{1,0} M \oplus T^{0,1} M .
$$

Set $\mathrm{D}_{1}:=T^{c} M$ and define, as above, the subbundles $\mathrm{D}_{j}:=\mathrm{D}_{1}+\left[\mathrm{D}_{1}, \mathrm{D}_{j-1}\right], j \geqslant 1$ of the real bundle $T M$. Thanks to the equality $D_{1}=\mathbb{C} \otimes \mathrm{D}_{1}$ and $\left[\mathbb{C} \otimes \mathrm{D}_{1}, \mathbb{C} \otimes \mathrm{D}_{j-1}\right]=\mathbb{C} \otimes\left[\mathrm{D}_{1}, \mathrm{D}_{j-1}\right]$, one verifies by means of a simple induction that $D_{j}=\mathbb{C} \otimes \mathrm{D}_{j}$ for each $j \geqslant 1$. Therefore, it is possible to restate the definition of total nondegeneracy in terms of the real distributions $\mathrm{D}_{j}$ as follows: $:^{2} M$ is a length $\rho$ totally nondegenerate submanifold of $\mathbb{C}^{1+k}$ if and only if its associated tangent bundle $T M$ can be generated by means of the minimum possible number of iterated Lie brackets between the generators $\mathrm{X}:=\operatorname{Re} \mathscr{L}$ and $\mathrm{Y}:=\operatorname{Im} \mathscr{L}$ of $\mathrm{D}_{1}=T^{c} M$, increasing maximally through the filtration:

$$
\mathrm{D}_{1} \varsubsetneqq \mathrm{D}_{2} \varsubsetneqq \cdots \varsubsetneqq \mathrm{D}_{\rho}=T M .
$$

Roughly speaking, $M$ is a length $\rho$ totally nondegenerate CR manifold whenever after setting $h_{1}:=\mathrm{x}$ and $h_{2}:=\mathrm{Y}$, the behavior of the above filtration and also Lie brackets between $\mathrm{x}, Y$ can be identified by (simple) words of lengths $\leq \rho$, belonging to the rank two (real) free Lie algebra.

Now, let us consider the existence of totally nondegenerate CR manifolds, a question which may arise naturally at this time. For a fixed positive integer $k$-which will take over the role of the codimension for the sought CR manifolds-let $\rho$ be the smallest length $\ell$ such that $\mathrm{n}_{\ell}:=\operatorname{dim} \mathscr{F}_{\ell} \geqslant 2+k$. Then by [20, Theorem 2.7], one finds a rank two real subdistribution $\mathrm{D}_{1}:=\langle\mathrm{X}, \mathrm{Y}\rangle$ of $T \mathbb{R}^{2+k}$, defined on a neighborhood $\Omega \subset \mathbb{R}^{2+k}$ of the origin such that:

(i) $\operatorname{dim} \mathrm{D}_{\ell}(0)=\mathrm{n}_{\ell}$ for each $\ell<\rho$ and

(ii) $\operatorname{dim} \mathrm{D}_{\rho}(0)=2+k$ or equivalently $\mathrm{D}_{\rho}(0)=T_{0} \mathbb{R}^{2+k}$,

where as above, $D_{j}:=D_{j-1}+\left[D_{1}, D_{j-1}\right]$ and where $D_{\ell}(0) \subset T_{0} \mathbb{R}^{2+k}$ is the image space of $\mathrm{D}_{j}$ at the origin. By definition [1, p. 74], the second property (ii) indicates that the origin $0 \in \mathbb{R}^{2+k}$ is a finite type point of the distribution $\mathrm{D}_{1}$ of the full type $2+k$. Since $\mathrm{D}_{\rho}$ has the maximum possible dimension at 0 , one finds a certain open subset $M$ of $\Omega$, including the origin, such that $\mathrm{D}_{\rho}(p)$ is again of the maximum possible dimension for each $p \in M$, i.e., $\mathrm{D}_{\rho}(p)=T_{p} \mathbb{R}^{2+k}$. Then $M$, as an open subset of $\mathbb{R}^{2+k}$, is a real submanifold of dimension $2+k$ and we claim that it is actually a CR generic submanifold of $\mathbb{C}^{1+k} \equiv \mathbb{R}^{2+2 k}$ of codimension $k$. Indeed, defining the complex structure map $J: \mathrm{D}_{1} \rightarrow \mathrm{D}_{1}$ on the subbundle $\mathrm{D}_{1}:=\left.\mathrm{D}_{1}\right|_{M}$ of $T M$ by $J(\mathrm{X})=\mathrm{Y}$ and $J(\mathrm{Y})=-\mathrm{X}$, the complexified bundle $\mathbb{C} \otimes \mathrm{D}_{1}$ can be decomposed as (cf. [13, p. 1573]):

$$
\mathbb{C} \otimes \mathrm{D}_{1}:=D^{1,0} \oplus D^{0,1}
$$

where $D^{1,0}$ and $D^{0,1}$ are generated by the single vector fields $\mathscr{L}:=\mathrm{X}+i \mathrm{Y}$ and $\overline{\mathscr{L}}:=\mathrm{X}-i \mathrm{Y}$, respectively. The rank one complex subbundle $D^{1,0} \subset \mathbb{C} \otimes T M$ is involutive. Then by definition [13], $\mathrm{D}_{1}$ is a $C R$ structure and the real submanifold $M$ is a CR manifold of $\mathrm{CR}$ dimension $1=\frac{1}{2} \operatorname{rank} \mathrm{D}_{1}$ and codimension $k=\operatorname{dim}_{\mathbb{R}} M-2 \mathrm{CRdim} M$. As before, we can denote $\mathrm{D}_{1}, D^{1,0}$ and $D^{0,1}$ by $T^{c} M, T^{1,0} M$ and $T^{0,1} M$, respectively. Moreover, as $M$ is an open subset of $\mathbb{R}^{2+k}$, then it is real analytic. Hence according to [21, Proposition 3.3] (expanded version) we can assume that $M$, regarded locally, is a generic submanifold of $\mathbb{C}^{1+k}$. Finally, two properties (i) and (ii) guarantee that $M$ is also totally nondegenerate.

${ }^{2}$ For technical reasons, we prefer to keep this definition in terms of the complexified distributions $D_{j}$ as Definition 1.1. 
By definition, on the other hand, for every arbitrary real analytic totally nondegenerate submanifold $M \subset \mathbb{C}^{1+k}$ of codimension $k$, passing through the origin and with $\mathrm{D}_{1}=T^{c} M$, the above two items (i) and (ii) are satisfied. This indicates that the so-called Hörmander numbers of $M$ (see [1] for definition) are 2, 3, 4, ., $\rho$ with the maximum possible multiplicities $\mathrm{m}_{2}, \mathrm{~m}_{3}, \ldots, \mathrm{m}_{\rho-1}, \mathrm{~m}_{\rho}^{\prime}$, respectively, where, $\mathrm{m}_{j}:=\mathrm{n}_{j}-\mathrm{n}_{j-1}$ for $j=1, \ldots, \rho-1$-as was in the case of the rank two free Lie algebra-and $\mathrm{m}_{\rho}^{\prime}:=k-\sum_{j=1}^{\rho-1} \mathrm{~m}_{j} \leqslant \mathrm{~m}_{\rho}$. Then, by summing up the results and applying Theorems 4.3.2 and 4.5.1 of [1], we can state that;

Theorem 1.1 (i) In each codimension $k$, totally nondegenerate real analytic CR generic submanifolds $M \subset \mathbb{C}^{1+k}$, passing through the origin, exist. The length $\rho$ of such manifolds is determined as the smallest integer $\ell$ such that $\mathrm{n}_{\ell} \geqslant 2+k$.

(ii) In agreement with the above notations, consider the canonical coordinates $\left(z, \mathbf{w}^{2}, \ldots\right.$, $\left.\mathbf{w}^{\rho-1}, \mathbf{w}^{\rho}\right)$ of $\mathbb{C}^{1+k}$, with $z \in \mathbb{C}$, with $\mathbf{w}^{j} \in \mathbb{C}^{\mathrm{m}_{j}}$ for $j=2, \ldots, \rho-1$ and with $\mathbf{w}^{\rho} \in \mathbb{C}^{\mathrm{m}_{\rho}^{\prime}}$. Assign the weight 1 to $z$ and the weight $j$ to each component of the vector $\mathbf{w}^{j}$ and to its real and imaginary parts, as well, for $j=1, \ldots, \rho$. Then, the already mentioned submanifolds $M \subset \mathbb{C}^{1+k}$ can be represented locally near the origin as the graph of some $k$ real analytic functions:

$$
\operatorname{Im} \mathbf{w}^{j}:=\boldsymbol{\Phi}_{j}\left(z, \bar{z}, \operatorname{Re} \mathbf{w}^{2}, \ldots, \operatorname{Re} \mathbf{w}^{j-1}\right)+\mathrm{O}(j) \quad(j=2, \ldots, \rho),
$$

where $\boldsymbol{\Phi}_{j}$ is a weighted homogeneous vector-valued polynomial of the weight $j$ and where $\mathrm{O}(j)$ is some certain (possibly vanishing) sum of monomials of weights $\geqslant j+1$. Moreover, denoting by $\boldsymbol{\Xi}_{j}:=\boldsymbol{\Phi}_{j}+\mathrm{O}(j)$, the right hand sides of the above equations, we have:

$$
\boldsymbol{\Xi}_{j}(0, \bar{z}, \operatorname{Re} \mathbf{w}) \equiv \boldsymbol{\Xi}_{j}(z, 0, \operatorname{Re} \mathbf{w}) \equiv 0
$$

In 2004, Valerii Beloshapka established in [5] his universal model surfaces associated with totally nondegenerate CR manifolds and designed an effective method to construct them. It was actually along the celebrated approach initiated first by Poincaré [25] in 1907 to study real submanifolds in the complex space $\mathbb{C}^{2}$ by means of the associated model surface, namely the Heisenberg sphere [22]. Several years later in 1974, Chern and Moser in their seminal work [9] notably developed this approach by associating appropriate models to nondegenerate real hypersurfaces in complex spaces. In this framework, many questions about automorphism groups, classification, invariants and others can be reduced to similar problems about the associated models.

To the best of author's knowledge, Beloshapka's work is the most general modelconstruction in the class of totally nondegenerate CR manifolds of arbitrary dimensions. Roughly speaking and after appropriate weight assignments (see Sect. 2 for more details), a Beloshapka's model of totally nondegenerate manifolds in CR dimension one (as is our specific case), codimension $k$ and accordingly determined length $\rho$ is represented as the graph of some weighted homogeneous polynomial functions of the form:

$$
\operatorname{Im} \mathbf{w}^{j}:=\boldsymbol{\Phi}_{j}\left(z, \bar{z}, \operatorname{Re} \mathbf{w}^{2}, \ldots, \operatorname{Re} \mathbf{w}^{j-1}\right) \quad(j=2, \ldots, \rho),
$$

obtained actually by removing the non-homogeneous parts $\mathrm{O}(j)$ from the general defining equations (3) of manifolds belonging to this class. Beloshapka's models are all homogeneous, of finite type and enjoy several other nice properties [5, Theorem 14] that exhibit their significance. Two totally nondegenerate CR manifolds are holomorphically equivalent whenever 
their associated models are as well. Moreover, they are most symmetric nondegenerate surfaces in the sense that dimension of the group of automorphisms (see below for definition) of a totally nondegenerate manifold does not exceed that of its associated model.

Convention 1.2 Let us stress that throughout this paper, we mainly deal with Beloshapka's totally nondegenerate $\mathrm{CR}$ generic models in CR dimension one which, for the sake of brevity, are also termed as "CR models" or "models". We fix the notation $M_{k}$ for such CR models in codimension $k$.

For a length $\rho$ CR model $M_{k} \subset \mathbb{C}^{1+k}$ in coordinates $\left(z, w_{1}, \ldots, w_{k}\right)$, a holomorphic vector field:

$$
X:=Z(z, w) \frac{\partial}{\partial z}+\sum_{l=1}^{k} W^{l}(z, w) \frac{\partial}{\partial w_{l}}
$$

is called an infinitesimal $C R$ automorphism whenever its real part is tangent to $M_{k}$, that is $\left.(\mathrm{X}+\overline{\mathrm{X}})\right|_{M_{k}} \equiv 0$. The collection of all infinitesimal CR automorphisms associated with $M_{k}$ form a Lie algebra, denoted by $\mathfrak{a u t}_{C R}\left(M_{k}\right)$. It is the CR symmetry Lie algebra of $M_{k}$ in the terminology of Sophus Lie's symmetry theory [18] and is of finite dimension, of polynomial type and graded of the form [5,31]:

$$
\mathfrak{a u t}_{C R}\left(M_{k}\right):=\underbrace{\mathfrak{g}_{-\rho} \oplus \cdots \mathfrak{g}_{-1}}_{\mathfrak{g}_{-}} \oplus \mathfrak{g}_{0} \oplus \underbrace{\mathfrak{g}_{1} \oplus \cdots \oplus \mathfrak{g}_{\varrho}}_{\mathfrak{g}_{+}}, \quad \varrho, \rho \in \mathbb{N},
$$

with $\left[\mathfrak{g}_{i}, \mathfrak{g}_{j}\right] \subset \mathfrak{g}_{i+j}$. Viewing the real analytic CR generic model $M_{k}$ in a purely intrinsic way, one may consider the local Lie group $\operatorname{Aut}_{C R}\left(M_{k}\right)$, associated with $\mathfrak{a u t}_{C R}\left(M_{k}\right)$, comprising automorphisms of the CR structure, namely of local $\mathscr{C}^{\infty}$ diffeomorphisms $h: M_{k} \rightarrow M_{k}$ satisfying:

$$
h_{*}\left(T^{c} M_{k}\right)=T^{c} M_{k} .
$$

In other words, $h$ belongs to Aut $C R\left(M_{k}\right)$ if and only if it is a (local) biholomorphism of $M_{k}$ [19]. Corresponding to (4), one may write:

$$
\operatorname{Aut}_{C R}\left(M_{k}\right):=\mathrm{G}_{-} \cdot \mathrm{G}_{0} \cdot \mathrm{G}_{+} \text {. }
$$

Beloshapka [5] showed that the Lie group $\mathrm{G}_{-}$associated with the above subalgebra $\mathfrak{g}_{-}$of $\mathfrak{a u t}_{C R}\left(M_{k}\right)$ is $(2+k)$-dimensional, acts on $M_{k}$ freely and can naturally be identified with $M_{k}$, itself. Also, $\mathrm{G}_{0}$ associated with the subalgebra $\mathfrak{g}_{0}$ comprises all linear automorphisms of $M_{k}$ in the isotropy subgroup $\operatorname{Aut}_{0}\left(M_{k}\right)$ of $\operatorname{Aut}_{C R}\left(M_{k}\right)$ at $0 \in M_{k}$ while $\mathrm{G}_{+}$, associated with $\mathfrak{g}_{+}$, comprises as well all the nonlinear ones.

Determining such Lie algebras of infinitesimal CR automorphisms is a question which lies pivotally at the heart of the problem of classifying local analytic CR manifolds up to biholomorphisms (see, e.g., [7] and the references therein). In fact, the groundbreaking works of Sophus Lie and his followers show that the most fundamental question in concern here is to draw up lists of possible such Lie algebras which would classify all possible manifolds according to their CR symmetries.

From a computational point of view, although computing the nonpositive part $\mathfrak{g}_{-} \oplus \mathfrak{g}_{0}$ of $\mathfrak{a u t}_{C R}\left(M_{k}\right)$ is partly convenient - in particular by means of the algorithm designed in [31] unfortunately for $\mathfrak{g}_{+}$one needs highly complicated computations which rely on constructing and solving arising systems of partial differential equations [21,30,32]. Nevertheless, after 
several years of experience in computing these algebras in various dimensions, Beloshapka [2] conjectured that; ${ }^{3}$

Conjecture 1.3 (Beloshapka's Maximum Conjecture) Each of Beloshapka's totally nondegenerate CR models $M$ of length $\rho \geq 3$ has holomorphic rigidity; that is: in its associated graded algebra $\mathfrak{a u t}_{C R}(M)$, the subalgebra $\mathfrak{g}_{+}$is trivial or equivalently $\varrho=0$ [cf. (4)].

Holding this conjecture true may bring about having several other facts about CR models and their associated totally nondegenerate CR manifolds (see, e.g., [3]). At present, there are only a few considerable results that verify it in some specific cases. For instance, Gammel and Kossovskiy [12] confirmed it in the specific length $\rho=3$. Kossovskiy also proved this conjecture in [14] for length four model CR manifolds with reflection. Both of these proofs are based on the structure of the envelopes of holomorphy of the corresponding models. Furthermore, the author in [27] provided a short proof for this conjecture in CR dimension one. In that paper, it is also shown that in each arbitrary length $\rho \geq 3$, there exists at least one CR model — called by full-model — with holomorphic rigidity. These results of [27] are proved by means of some certain facts arisen in the Tanaka theory of prolongations. Two more relevant (partial) results in this setting are as follows:

- if $\rho=4$, then $\varrho \leqslant 1$ [6, Corollary 7],

- if $\rho=5$, then $\varrho \leqslant k$, where $k$ is the CR codimension of $M$ [34, Proposition 2.2].

In these works the results are achieved by means of directly computing the associated desired Lie algebras. But the difficulty of this method which lies in the incredible differentialalgebraic complexity involved (cf. [30]), may convince one to also consider this conjecture through other ways.

On the other hand, recently in [21] and in particular in Sect. 5 of that paper, we attempted to study by means of Cartan's classical approach, the biholomorphic equivalence problem to the 5-dimensional length 3 cubic model $M_{3} \subset \mathbb{C}^{4}$-denoted there by $M_{\mathrm{c}}^{5}$-represented as the graph of three defining equations:

$$
\operatorname{Im} w_{1}=z \bar{z}, \quad \operatorname{Im} w_{2}=z \bar{z}(z+\bar{z}), \quad \operatorname{Im} w_{3}=z \bar{z}(z-\bar{z}) .
$$

Just as a consequence of the results, we observed that the associated 7-dimensional CR automorphism algebra:

$$
\mathfrak{a u t}_{C R}\left(M_{3}\right):=\mathfrak{g}_{-3} \oplus \mathfrak{g}_{-2} \oplus \mathfrak{g}_{-1} \oplus \mathfrak{g}_{0},
$$

computed in Sect. 3 of that paper, is surprisingly isomorphic to that defined by the final constant type structure equations of the already mentioned equivalence problem (cf. [21, Theorem 5.1]). This observation was our original motivation to look upon Cartan's classical approach as an appropriate way to study Beloshapka's maximum conjecture. Examining this idea on some other CR models like those studied in [22,24,29,32] also convinced us more about its effectiveness. Indeed, the systematic approach developed in recent years by Joël Merker, Samuel Pocchiola and the present author provides a unified way toward treating the wide variety of biholomorphic equivalence problems between CR manifolds.

Cartan's classical method for solving equivalence problems includes three major parts: absorption, normalization and prolongation. In the CR context, usually all steps require advanced computations, the size of which increases considerably as soon as the dimension of CR manifolds increases, even by one unit. In particular, among the absorption-normalization

3 Although Beloshapka introduced his conjecture in 2012 but he and his students had been aware of it since several years before (see, e.g., [3,12]). 
steps, one encounters some arising polynomial systems, the solutions of which determine the value of some group parameters associated with the problem. As is quite predictable due to the arbitrariness of dimension, one of our main obstacles for solving the equivalence problem, under question, will be actually solving these arising polynomial systems in this general manner. In order to bypass and manipulate this critical complexity, our main weapon in this paper is in fact some helpful results achieved by a careful weight analysis of the equivalence problems, under study. Such analysis enables us to provide a much more convenient weighted homogeneous subsystem of the already mentioned system which is deceptively hidden inside the original one and opens our way of finding the desired general outcome of the normalization process.

This paper is organized as follows. In the next preliminary section, Sect. 2, we present a brief description of constructing defining equations of Beloshapka's CR models in CR dimension one. Next in Sect. 3, we endeavor to find certain expression of the structure equations associated with the biholomorphic equivalence problem between an arbitrary length $\rho$ CR model $M_{k}$ and any arbitrary totally nondegenerate CR manifold $\mathbf{M}_{k}$ of the same length and codimension (cf. Theorem 1.1). For this purpose, first we construct, in an almost explicit manner, an initial frame:

$$
\mathbb{L}:=\left\{\mathscr{L}_{1,1}, \mathscr{L}_{1,2}, \mathscr{L}_{2,3}, \ldots, \mathscr{L}_{\rho, 2+k}\right\}
$$

on $\mathbb{C} \otimes T M_{k}$, where $\mathscr{L}_{1,1}$ and $\mathscr{L}_{1,2}=\overline{\mathscr{L}_{1,1}}$ are the single generators of $T^{1,0} M_{k}$ and $T^{0,1} M_{k}$, respectively, and where each $\mathscr{L}_{\ell, i}$ is a length $\ell$ iterated Lie bracket between them constructed as a simple word. We also consider a so-called lifted frame $\mathbf{L}:=\left\{\mathbf{L}_{1,1}, \mathbf{L}_{1,2}, \ldots, \mathbf{L}_{\rho, 2+k}\right\}$ on $\mathbb{C} \otimes T \mathbf{M}_{k}$, constructed in a similar manner as simple words written by the single generators $\mathbf{L}_{1,1}$ and $\mathbf{L}_{1,2}:=\overline{\mathbf{L}_{1,1}}$. Let $\Sigma:=\left\{\sigma_{1,1}, \sigma_{1,2}, \ldots, \sigma_{\rho, 2+k}\right\}$ and $\Gamma:=\left\{\Gamma_{1,1}, \Gamma_{1,2}, \ldots, \Gamma_{\rho, 2+k}\right\}$ be two coframes on $\mathbb{C} \otimes T^{*} M_{k}$ and $\mathbb{C} \otimes T^{*} \mathbf{M}_{k}$, dual to the frames $\mathbb{L}$ and $\mathbf{L}$, respectively. We realize that for a general biholomorphic equivalence map $h: M_{k} \rightarrow \mathbf{M}_{k}$, the associated matrix of the induced complexified linear pull-back $h^{*}: \mathbb{C} \otimes T^{*} \mathbf{M}_{k} \rightarrow \mathbb{C} \otimes T^{*} M_{k}$, expressed in terms of the coframes $\Gamma$ and $\Sigma$ is an invertible $(2+k) \times(2+k)$ lower triangular matrix:

$$
\mathbf{g}:=\left(\begin{array}{cccccc}
a_{1}^{p} \bar{a}_{1}^{q} & \ldots & 0 & 0 & 0 & 0 \\
\vdots & \vdots & \vdots & \vdots & \vdots & \vdots \\
a_{\bullet} & \ldots & a_{3} & a_{1} \bar{a}_{1} & 0 & 0 \\
a_{\bullet} & \ldots & a_{5} & -\bar{a}_{2} & \bar{a}_{1} & 0 \\
a_{\bullet} & \ldots & a_{4} & a_{2} & 0 & a_{1}
\end{array}\right) \quad \text { with } a_{1} \neq 0
$$

for some certain complex-valued functions $a_{1}, a_{2}, a_{3}, \ldots$, in terms of coordinates of $\mathbb{C}^{1+k}$. Only some powers of $a_{1}$ and $\bar{a}_{1}$ are visible at the diagonal of $\mathbf{g}$. As is standard in the terminology of Cartan's theory, we call this matrix the ambiguity matrix (or G-structure) of the mentioned equivalence problem and its nonzero entries $a_{\bullet}$ the group parameters. The collection of all such matrices forms a Lie group $G$ which is called the structure Lie group of the equivalence problem.

The main focus of Sect. 4 is a weight analysis on the structure equations, constructed in the preceding section by applying necessary differentiations and computations on the already obtained equality $\Gamma=\mathbf{g} \cdot \Sigma$. In particular, after appropriate weight assignment to the appearing group parameters and also after inspecting carefully the inverse of the ambiguity matrix $\mathbf{g}$, we discover that all the torsion coefficients appearing through the structure equations are of the same weight zero (cf. Proposition 4.4).

Next in Sect. 5, we consider the outcome of the absorption and normalization steps on the constructed structure equations. It is in this section that we extract a subtle weighted 
homogeneous subsystem of the polynomial system, arising among the absorption and normalization steps. Solving this subsystem by means of some computational techniques from weighted algebraic geometry [11], we conclude that except $a_{1}$, all the other group parameters $a_{2}, a_{3}, \ldots$ must be vanishing;

Proposition 1.4 (cf. Proposition 5.5) All the appearing group parameters $a_{2}, a_{3}, \ldots$ vanish identically after sufficient steps of absorption and normalization.

This key result converts our structure equations into a delicate constant type form (cf. Proposition 5.6):

$$
d \Gamma_{\ell, i}:=\left(p_{i} \alpha+q_{i} \bar{\alpha}\right) \wedge \Gamma_{\ell, i}+\sum_{\substack{l+m=\ell \\ j, n}} c_{j, n}^{i} \Gamma_{l, j} \wedge \Gamma_{m, n} \quad(\ell=1, \ldots, \rho, i=1, \ldots, 2+k) .
$$

Here, $p_{i}, q_{i}, c_{j, n}^{i}$ are some constant integers and $\alpha:=\frac{d a_{1}}{a_{1}}$ is the only remaining MaurerCartan form. For the only not-yet-determined parameter $a_{1}$, we discover that it is either normalizable to a real (or imaginary) group parameter or it is never normalizable. We also provide a simple criterion, concerning this normalization (cf. Proposition 5.7). In the former case, the structure group $G$ of the above ambiguity matrices will be reduced to $G^{\text {red }}$ of real dimension 1 while in the later case $G^{\text {red }}$ is of real dimension 2. Next, we start the last part, namely prolongation, of Cartan's method. Accordingly, the original equivalence problem to our model $M_{k}$ converts by that to the prolonged space $M_{k} \times G^{\text {red }}$ of real dimension either $3+k$ or $4+k$. Finding the structure equations of this new equivalence problem is easy, it suffices adding the equation $d \alpha=0$ to the above structure equations (6). This provides us with the following main result of this paper;

Theorem 1.2 (cf. Theorem 5.1) The biholomorphic equivalence problem of a totally nondegenerate CR model $M_{k}$ of codimension $k$ and real dimension $2+k$ is reducible to some absolute parallelisms, namely to some certain $\{e\}$-structures on prolonged manifolds of real dimension either $3+k$ or $4+k$.

In the short Sect. 6, we prove the maximum conjecture 1.3 in $\mathrm{CR}$ dimension one as a consequence of the achieved results. According to the principles of Cartan's theory [23], once we receive the final constant type structure equations of the equivalence problem to each CR model $M_{k}$, we can plainly attain the structure of its symmetry Lie algebra $\mathfrak{a u t}_{C R}\left(M_{k}\right)$. Computing this algebra, we realize that it is graded without any positive part as was the assertion of the maximum conjecture.

Proposition 1.5 (cf. Proposition 6.1) The Lie algebra $\mathfrak{a u t}_{C R}\left(M_{k}\right)$ of a k-codimensional weight $\rho$ totally nondegenerate model $M_{k}$, of $C R$ dimension one, is graded of the form:

$$
\mathfrak{a u t}_{C R}\left(M_{k}\right):=\underbrace{\mathfrak{g}_{-\rho} \oplus \cdots \oplus \mathfrak{g}_{-1}}_{\mathfrak{g}_{-}} \oplus \mathfrak{g}_{0}
$$

where $\mathfrak{g}_{-}$is $(2+k)$-dimensional and where $\mathfrak{g}_{0}$ is Abelian of dimension either 1 or 2 . Thus, we have:

$$
\operatorname{dim}\left(\mathfrak{a u t}_{C R}\left(M_{k}\right)\right)=3+k \text { or } 4+k .
$$

As mentioned, one finds in [27] a very shorter proof of the maximum conjecture in CR dimension one based upon some results and techniques in the Tanaka theory of transitive 
prolongations. However, it is extremely important to notice that solving biholomorphic equivalence problem to totally nondegenerate models of CR dimension one, as is the main goal of this paper, not only helps us to reconfirm the maximum conjecture in this CR dimension but it also determines precisely desired invariants of the problem and the structure of associated algebras of infinitesimal CR automorphisms. Moreover, it provides one with the opportunity of computing moduli spaces $[4,16,29]$ of the models and constructing Cartan geometries on totally nondegenerate CR manifolds $[22,33]$. None of such results and opportunities are achieved in [27].

Finally in "Appendix A", we illustrate the results by considering the 8-dimensional length four CR model $M_{6}$.

As a homogeneous space, each CR model $M_{k}$ can be considered as a quotient space [see the paragraph after Eq. (5)]:

$$
M_{k} \equiv \frac{\operatorname{Aut}_{C R}\left(M_{k}\right)}{\operatorname{Aut}_{0}\left(M_{k}\right)} \cong \mathrm{G}_{-}
$$

of the CR automorphism group Aut $\operatorname{Ar}_{C R}\left(M_{k}\right)$, corresponding to $\mathfrak{a u t}_{C R}\left(M_{k}\right)$ by its isotropy subgroup $\operatorname{Aut}_{0}\left(M_{k}\right)$ at the origin, corresponding to $\mathfrak{g}_{0} \oplus \mathfrak{g}_{+}$. As the above proposition states, such isotropy group is just $\mathrm{G}_{0}$, corresponding to the Abelian algebra $\mathfrak{g}_{0}$ and comprises only linear CR automorphisms $h: M_{k} \rightarrow M_{k}$, preserving the origin. Even more precisely, in this case that $\operatorname{dim} \mathfrak{g}_{0}$ is either 1 or 2 , then $G_{0}$ can be identified with the matrix Lie group $\mathrm{GL}(1, \mathbb{R})=\left(\mathbb{R}^{*}, \times\right)$ in the former case and $\mathrm{GL}(1, \mathbb{C})=\left(\mathbb{C}^{*}, \times\right)$ in the latter.

\section{Beloshapka's models}

In this preliminary section, we explain the method of constructing defining equations of Beloshapka's models in $\mathrm{CR}$ dimension one. For more detailed explanation, we refer the reader to [5]. In each fixed CR codimension $k$, a certain Beloshapka's model $M_{k} \subset \mathbb{C}^{1+k}$ can be represented in coordinates $\left(z, w_{1}, \ldots, w_{k}\right)$ as the graph of some $k$ real-valued polynomial functions, which roughly speaking, are the homogeneous parts of the defining equations introduced in Theorem 1.1. Throughout constructing these defining polynomials and to each complex coordinate $\mathrm{x}$, it will be assigned a weight number $[\mathrm{x}]$. Recall that for a monomial $\mathrm{x}_{1}^{\alpha_{1}} \cdots \mathrm{x}_{n}^{\alpha_{n}}$, the associated weight is defined as $\sum_{i=1}^{n} \alpha_{i}\left[\mathrm{x}_{i}\right]$. Moreover, a polynomial is called weighted homogeneous of the weight $\mathrm{w}$ whenever all of its monomials are of this weight. We assign the same weight $[\mathrm{x}]$ to the conjugation $\bar{x}$ and real and imaginary parts of $\mathrm{x}$, as well. Before starting the construction, we first need the following definition;

Definition 2.1 (cf. [15]) An arbitrary $\mathscr{C}^{2}$ complex function $f: \Omega \subset \mathbb{C}^{n} \rightarrow \mathbb{C}$ in terms of the coordinates $\left(z_{1}, \ldots, z_{n}\right)$ is called pluriharmonic on its domain $\Omega$ whenever for each $i, j=1, \ldots, n$ we have:

$$
\frac{\partial^{2} f}{\partial \mathbf{z}_{i} \partial \overline{\mathbf{z}}_{j}} \equiv 0 .
$$

In the case that $f$ is real-valued, then locally, pluriharmonicity of $f$ is equivalent to state that it is the real part of a holomorphic function [15, Propoition 2.2.3].

By convention, we assign to the complex variable $z$ the weight $[z]=1$. The weights of the next complex variables $w_{1}, w_{2}, \ldots$, which are absolutely bigger than 1 , will be determined as follows, step by step. At the first onset that only the weight of the single variable $z$ is known, let $\mathscr{N}_{2}$ be a basis for the space of all non-pluriharmonic real-valued polynomials of 
the homogeneous weight 2 , in terms of the complex variables $z$ and $\bar{z}$. A careful inspection shows that $\mathscr{N}_{2}$ comprises merely the single term:

$$
\mathscr{N}_{2}:=\{z \bar{z}\} .
$$

Since the cardinality of this set is $k_{2}=1$, then we assign immediately the weight 2 to the next one complex variable $w_{1}$, i.e., $\left[w_{1}\right]=2$.

At the moment, two of the complex variables $z$ and $w_{1}$ have received their weight numbers. Define the next collection $\mathscr{N}_{3}$ as a basis for the space of all real-valued polynomials of the weight 3 , in terms of the variables $z, \bar{z}$ and $\operatorname{Re} w_{1}$, which are non-pluriharmonic on the submanifold represented by the weight two homogeneous polynomial $\operatorname{Im} w_{1}=z \bar{z}$ in $\mathbb{C}^{2}$. Again, a careful inspection shows that:

$$
\mathscr{N}_{3}:=\left\{\operatorname{Re} z^{2} \bar{z}=\frac{z^{2} \bar{z}+z \bar{z}^{2}}{2}, \quad \operatorname{Im} z^{2} \bar{z}=\frac{z^{2} \bar{z}-z \bar{z}^{2}}{2 i}\right\} .
$$

This time, since the cardinality of $\mathscr{N}_{3}$ is $k_{3}=2$, then immediately we assign the weight 3 -namely the weight of the monomials in $\mathscr{N}_{3}$ - to the next two complex variables $w_{2}$ and $w_{3}$.

Inductively, assume that $\mathscr{N}_{j_{0}}$ is the last constructed basis for some integer $j_{0} \in \mathbb{N}$. This means that all the complex variables $z, w_{1}, w_{2}, w_{3}, \ldots, w_{\mathrm{r}}$ have received their weight numbers where $\mathrm{r}:=\sum_{i=2}^{j_{0}} k_{i}$ and where $k_{i}:=$ Card $\mathscr{N}_{i}$. To construct the next collection $\mathscr{N}_{j_{0}+1}$ and for the sake of clarity, let us show the $k_{i}$ elements of each $\mathscr{N}_{i}$ as $\mathscr{N}_{i}:=\left\{\mathrm{t}_{1}^{i}, \mathrm{t}_{2}^{i}, \ldots, \mathrm{t}_{k_{i}}^{i}\right\}$. Also for each $\ell=2, \ldots, j_{0}$, let $\mathbf{w}^{\ell}=\left(w_{l}, \ldots, w_{l+k_{\ell}-1}\right)^{t}$ be the $k_{\ell}$-tuple of all complex variables $w_{1}, \ldots, w_{\mathrm{r}}$ of the same weight $\ell$ and consider:

$$
A_{\ell}=\left(\begin{array}{ccc}
a_{11}^{\ell} & \ldots & a_{1 k_{\ell}}^{\ell} \\
\vdots & \vdots & \vdots \\
a_{k_{\ell} 1}^{\ell} & \ldots & a_{k_{\ell} k_{\ell}}^{\ell}
\end{array}\right)
$$

as some real $k_{\ell} \times k_{\ell}$ matrix of the maximum $\operatorname{Rank}\left(A_{\ell}\right)=k_{\ell}$. Then, the sought collection $\mathscr{N}_{j_{0}+1}$ is defined as a basis for the space of all real-valued polynomials of the weight $j_{0}+1$, in terms of the already weight determined variables $z, \bar{z}, \operatorname{Re} w_{1}, \operatorname{Re} w_{2}, \ldots, \operatorname{Re} w_{\mathrm{r}}$, which are non-pluriharmonic on the submanifold represented as the graph of some $r$ weighted homogeneous polynomial functions:

$$
\operatorname{Im} \mathbf{w}^{\ell}=A_{\ell} \cdot\left(\begin{array}{c}
\mathrm{t}_{1}^{\ell} \\
\vdots \\
\mathrm{t}_{k_{\ell}}^{\ell}
\end{array}\right) \quad\left(\ell=2, \ldots, j_{0}\right),
$$

in $\mathbb{C}^{\mathrm{r}+1}$. Here, Im $\mathbf{w}^{\ell}$ is the $k_{\ell}$-tuple of imaginary parts of $\mathbf{w}^{\ell}$. If the cardinality of $\mathscr{N}_{j_{0}+1}$ is $k_{j_{0}+1}$, then one assigns immediately the weight $j_{0}+1$ to all the next complex variables $w_{\mathrm{r}+1}, \ldots, w_{\mathrm{r}+k_{j_{0}+1}}$.

\subsection{Constructing the defining equations}

After assigning appropriate weights to the complex variables $z, w_{\bullet}$, we are ready to explain the procedure of constructing defining polynomials of a $k$-codimensional Beloshapka's model $M_{k} \subset \mathbb{C}^{k+1}$. In this case, we need only the assigned weights to the complex coordinates 
$\left(z, w_{1}, \ldots, w_{k}\right)$ of $M_{k}$, hence we have to construct the above sets $\mathscr{N}_{i}$ until we arrive at the stage $i=\rho$ where $\rho$ is the smallest integer satisfying:

$$
k \leqslant k_{2}+\cdots+k_{\rho-1}+k_{\rho}
$$

In this case, the chain of associated weights to the complex variables $z, w_{1}, \ldots, w_{k}$ is ascending and the last variable $w_{k}$ is of the maximum weight $\rho$, which will be in fact the length of $M_{k}$ (cf. Theorem 1.1).

Now, for each $\ell=2, \ldots, \rho-1$, consider the $k_{\ell}$-tuple $\mathbf{w}^{\ell}$ and the $k_{\ell} \times k_{\ell}$ matrix $A_{\ell}$ as above. For $\ell=\rho$ and since in this case the number of the present weight $\rho$ variables among $w_{1}, \ldots, w_{k}$ is $m=k-\sum_{i=2}^{\rho-1} k_{i} \leqslant k_{\rho}$, then consider the $m$-tuple $\mathbf{w}^{\rho}$ as $\mathbf{w}^{\rho}=$ $\left(w_{k-m+1}, \ldots, w_{k}\right)$. Also let:

$$
A_{\rho}=\left(\begin{array}{ccc}
a_{11}^{\rho} & \ldots & a_{1, k_{\rho}}^{\rho} \\
\vdots & \vdots & \vdots \\
a_{m 1}^{\rho} & \ldots & a_{m k_{\rho}}^{\rho}
\end{array}\right)
$$

be a certain real $m \times k_{\rho}$ matrix of the maximum $\operatorname{Rank}\left(A_{\rho}\right)=m$. Then, the desired defining equations of $M_{k}$ can be represented in the following matrix form:

$$
\operatorname{Im} \mathbf{w}^{\ell}=A_{\ell} \cdot\left(\begin{array}{c}
\mathrm{t}_{1}^{\ell} \\
\vdots \\
\mathrm{t}_{k_{\ell}}^{\ell}
\end{array}\right), \quad(\ell=2, \ldots, \rho) .
$$

As we observe, in a fixed codimension $k$ one may find infinite number of CR models $M_{k}$ determined by different values of the above matrix entries $a_{i j}^{\ell}$. Nevertheless, possibly many of them are equivalent, up to some biholomorphic change of coordinates. For example in codimension $k=3, \mathrm{CR}$ models $M_{3} \subset \mathbb{C}^{4}$ are represented as the graph of some three defining polynomials:

$$
\begin{aligned}
& \operatorname{Im} w_{1}=a z \bar{z}, \\
& \operatorname{Im} w_{2}=a_{11}\left(z^{2} \bar{z}+z \bar{z}^{2}\right)+i a_{12}\left(z^{2} \bar{z}-z \bar{z}^{2}\right), \quad\left(a, a_{i j} \in \mathbb{R}\right), \\
& \operatorname{Im} w_{3}=a_{21}\left(z^{2} \bar{z}+z \bar{z}^{2}\right)+i a_{22}\left(z^{2} \bar{z}-z \bar{z}^{2}\right) .
\end{aligned}
$$

However, by some simple biholomorphic changes of coordinates like those presented at the page 50 of [21] (expanded version), one shows that they are biholomorphically equivalent to the so-called 5-cubic model:

$$
M_{\mathrm{c}}^{5}: \quad\left[\begin{array}{l}
\operatorname{Im} w_{1}=z \bar{z} \\
\operatorname{Im} w_{2}=z^{2} \bar{z}+z \bar{z}^{2}, \\
\operatorname{Im} w_{3}=i\left(z^{2} \bar{z}-z \bar{z}^{2}\right) .
\end{array}\right.
$$

Anyway, in this paper we do not stress on such biholomorphic normalizations since it will not matter whether the under consideration defining equations are normalized or not.

Summing up the above procedure, each arbitrary CR model $M_{k} \subset \mathbb{C}^{1+k}$ of codimension $k$ and of the length $\rho$ can be represented as the graph of some $k$ certain real-valued defining 
equations:

$$
M_{k}:\left\{\begin{aligned}
w_{1}-\bar{w}_{1} & =2 i \Phi_{1}(z, \bar{z}) \\
\vdots & \\
w_{j}-\bar{w}_{j} & =2 i \Phi_{j}(z, \bar{z}, w, \bar{w}) \\
\vdots & \\
w_{k}-\bar{w}_{k} & =2 i \Phi_{k}(z, \bar{z}, w, \bar{w}),
\end{aligned}\right.
$$

where each $\Phi_{j}$ is of the weight $\left[w_{j}\right]$, in terms of the complex variables $z, \bar{z}$ and real variables $\operatorname{Re} w_{i}$ with $\left[w_{i}\right] \supsetneqq\left[w_{j}\right]$. As one observes, the defining equations of $M_{k}$ are actually those of a certain $(k-1)$-codimensional model $M_{k-1}$, added just by the last equation $w_{k}-\bar{w}_{k}=$ $2 i \Phi_{k}(z, \bar{z}, w, \bar{w})$.

Remark 2.2 Instead of the above Beloshapka's algebraic method for constructing defining equations of a totally nondegenerate CR model $M_{k}$, Merker [17] has introduced a more geometric way by considering the affect of the total nondegeneracy on the converging power series expansions of the desired defining equations.

\section{Constructing associated structure equations}

Studying equivalences between geometric objects by means of Cartan's classical approach entails first some preliminary equipments, the end of them is the construction of associated structure equations. In the current case of biholomorphic equivalence to CR models, we follow the systematic method developed among the recent years in [21,24,29,32] which includes three major steps to bring us to the stage of constructing the sought structure equations:

- Finding appropriate initial CR frame for each model and computing its Lie commutators.

- Passage to the dual CR coframe and computing the associated Darboux-Cartan structure.

- Finding the ambiguity matrix of the equivalence problem, in question.

\subsection{Associated initial frames for the complexified tangent bundles}

In the defining equations (9) of a $k$-codimensional CR model $M_{k} \subset \mathbb{C}^{k+1}$ in coordinates $\left(z, w_{1}, \ldots, w_{k}\right)$, each real-valued polynomial $\Phi_{j}(z, \bar{z}, \bar{w})$ is $\mathrm{O}(2)$ and thus we can apply the analytic implicit function theorem in order to solve these equations for the $k$ variables $w_{j}$, $j=1, \ldots, k$. Performing this, we obtain equivalently a collection of $k$ complex defining equations like:

$$
M_{k}:\left\{w_{j}=\Theta_{j}(z, \bar{z}, \bar{w}) \quad(j=1, \ldots, k),\right.
$$

where each complex-valued polynomial function $\Theta_{j}$ is in terms of $z, \bar{z}, \bar{w}_{j}$ and some other conjugated variables $\bar{w}_{\bullet}$ of absolutely lower weights than $\left[w_{j}\right]$. By an induction on the weights associated with the complex coordinates $w_{1}, \ldots, w_{k}$, one verifies that similar to the real-valued functions $\Phi_{\bullet}$ also each complex-valued polynomial $\Theta_{j}$ is weighted homogeneous of the weight $\left[w_{j}\right]$.

Having in hand the complex defining polynomials (10) of the CR model $M_{k}$ and according to [20,21], then the associated holomorphic and antiholomorphic tangent bundles $T^{1,0} M_{k}$ 
and $T^{0,1} M_{k}$, can be generated, respectively, by the single vector fields:

$$
\mathscr{L}:=\frac{\partial}{\partial z}+\sum_{j=1}^{k} \frac{\partial \Theta_{j}}{\partial z}(z, \bar{z}, \bar{w}) \frac{\partial}{\partial w_{j}} \text { and } \overline{\mathscr{L}}:=\frac{\partial}{\partial \bar{z}}+\sum_{j=1}^{k} \frac{\partial \bar{\Theta}_{j}}{\partial \bar{z}}(z, \bar{z}, w) \frac{\partial}{\partial \bar{w}_{j}} .
$$

For $\mathrm{x}$ to be one of the complex variables $z, w_{1}, \ldots, w_{k}$, or one of their conjugations, or one of their real or imaginary parts, we assign the weight $-[x]$ to the standard vector filed $\frac{\partial}{\partial \mathrm{x}}$. Notice that for a weighted homogeneous polynomial $F(\mathrm{x}, \overline{\mathrm{x}})$, each differentiation of the shape $F_{\mathrm{x}_{i}}\left(\right.$ or $\left.F_{\overline{\mathrm{x}}_{i}}\right)$ decreases its weight by $\left[\mathrm{x}_{i}\right]$ numbers, if it does not vanish. Then, by a glance on the above expressions of $\mathscr{L}$ and $\overline{\mathscr{L}}$ one finds them as two weighted homogeneous fields of the same weight -1 .

\subsubsection{Notations}

Henceforth and in order to stress their lengths (and weights), let us denote by $\mathscr{L}_{1,1}$ and $\mathscr{L}_{1,2}$ the above vector field $\mathscr{L}$ and its conjugation $\overline{\mathscr{L}}$, respectively. By the total nondegeneracy of our length $\rho$ fixed CR model $M_{k}$, one constructs the sought initial frame on $\mathbb{C} \otimes T M_{k}$ by applying the iterated Lie brackets — or simple words in the terminology of free Lie algebrasof these two vector fields, up to the length $\rho$. Let us denote by $\mathscr{L}_{\ell, i}$ and call it by the $i$ th initial vector field, the $i$ th appearing independent vector filed obtained as an iterated Lie bracket of the length $\ell$. For example, the next and third appearing vector filed can be computed as the length two iterated Lie bracket:

$$
\mathscr{L}_{2,3}=\left[\mathscr{L}_{1,1}, \mathscr{L}_{1,2}\right]
$$

In the case that the reference to the order $i$ of a length $\ell$ initial vector field $\mathscr{L}_{\ell, i}$ is superfluous and by abuse of notation, we denote it just by $\mathscr{L}_{\ell}$, which actually is a vector field expressible (inductively) as:

$$
\mathscr{L}_{\ell}:=[\mathscr{L}_{1, i_{1}}, \underbrace{\left[\mathscr{L}_{1, i_{2}},\left[\ldots,\left[\mathscr{L}_{1, i_{\ell-1}}, \mathscr{L}_{1, i_{\ell}}\right]\right]\right]}_{\mathscr{L}_{\ell-1}}] \quad\left(i_{j}=1,2, \ell=1, \ldots, \rho\right) .
$$

Notice that according to the expressions of $\mathscr{L}_{1,1}$ and $\mathscr{L}_{1,2}$ in (11) and from the length $\ell=2$ to the end, one does not see any coefficient of $\frac{\partial}{\partial z}$ or $\frac{\partial}{\partial \bar{z}}$ in the expression of $\mathscr{L}_{\ell}$.

Lemma 3.1 Each length $\ell$ initial vector field $\mathscr{L}_{\ell}$ is homogeneous of the weight $-\ell$ with polynomial coefficients. Moreover, for two initial vector fields $\mathscr{L}_{\alpha, i}$ and $\mathscr{L}_{\beta, j}$ with $\alpha+\beta=\ell$, if $\left[\mathscr{L}_{\alpha, i}, \mathscr{L}_{\beta, j}\right] \not \equiv 0$ then it is a weighted homogeneous vector field of the weight $-\ell$, again with polynomial coefficients.

Proof Since the coefficients of the basis vector fields $\mathscr{L}_{1}$ are of polynomial type, the polynomiality of the coefficients in their iterated brackets is obvious. Concerning the weights, we continue by a plain induction on the length $\ell$. As we saw, the two vector fields $\mathscr{L}_{1,1}$ and $\mathscr{L}_{1,2}$ of the length $\ell=1$ are of the homogeneous weight -1 . For the next lengths and as our induction hypothesis, assume that all length $\ell$ vector fields:

$$
\mathscr{L}_{\ell}:=\sum_{\left[w_{i}\right] \geq \ell} \varphi_{i}(z, \bar{z}, w, \bar{w}) \frac{\partial}{\partial w_{i}}+\sum_{\left[w_{i}\right] \geq \ell} \psi_{i}(z, \bar{z}, w, \bar{w}) \frac{\partial}{\partial \bar{w}_{i}}
$$

are weighted homogeneous of the weight $-\ell$. Thus, the nonzero polynomial coefficients $\varphi_{i}$ and $\psi_{i}$ are homogeneous of the nonnegative weights $\left[w_{i}\right]-\ell$. Now, consider an arbitrary 
new appearing initial field $\mathscr{L}_{\ell+1}=\left[\mathscr{L}_{1}, \mathscr{L}_{\ell}\right]$ of the length $\ell+1$. Applying the Leibniz rule on this bracket with the present expressions of the weighted homogeneous fields $\mathscr{L}_{1}$ in (11) and $\mathscr{L}_{\ell}$, it manifests itself as a weight $-(\ell+1)$ homogeneous vector field, as was expected. The proof of the second part of the assertion is completely similar.

Proposition 3.2 Let $\mathscr{L}_{\alpha, i}$ and $\mathscr{L}_{\beta, j}$ be two initial vector fields associated with a length $\rho C R$ model $M_{k}$ with $\alpha+\beta=\ell$. Then, we have:

$$
\left[\mathscr{L}_{\alpha, i}, \mathscr{L}_{\beta, j}\right]=\sum_{t} \mathrm{c}_{t} \mathscr{L}_{\ell, t}
$$

for some constant integers $\mathrm{c}_{t}$. In particular if $\ell>\rho$, then $\left[\mathscr{L}_{\alpha, i}, \mathscr{L}_{\beta, j}\right] \equiv 0$.

Proof Since our length $\rho$ CR model $M_{k}$ is totally nondegenerate and according to the discussion after Definition 1.1, one verifies that the equality holds in the case that $\ell=1, \ldots, \rho-1$. Hence, let us prove the assertion first by assuming $\ell=\rho$. If $\left[\mathscr{L}_{\alpha, i}, \mathscr{L}_{\beta, j}\right]$ vanishes, then it remains nothing to prove. Otherwise, Lemma 3.1 indicates that this Lie bracket produces a weighted homogeneous vector field of the weight $-\rho$ with polynomial coefficients. Taking into account that the maximum weight of the extant complex variables is $\rho$, then this bracket can be written just in the form:

$$
\left[\mathscr{L}_{\alpha, i}, \mathscr{L}_{\beta, j}\right]:=\sum_{\left[w_{i}\right]=\rho} \mathrm{a}_{i} \frac{\partial}{\partial w_{i}}+\sum_{\left[w_{i}\right]=\rho} \mathrm{b}_{i} \frac{\partial}{\partial \bar{w}_{i}},
$$

for some constant integers $\mathrm{a}_{i}$ and $\mathrm{b}_{i}$. On the other hand, the projection map $\pi: \mathbb{C}^{1+k} \rightarrow$ $\mathbb{R}^{2+k}$ defined as $(x, y, \mathbf{u}, \mathbf{v}) \mapsto(x, y, \mathbf{u})$ constitutes a natural local chart-map on the real submanifold $M_{k}$-still we denote $z=x+i y$ and $w_{j}=u_{j}+i v_{j}$. Then, one restates intrinsically the expression of each $\mathscr{L}_{\ell}$ in terms of $z, \bar{z}, u_{j}$ by dropping $\frac{\partial}{\partial v_{j}}$ for $j=1, \ldots, k$ and also replacing each $v_{j}$ by its expression in (9). Then in particular, each weight $-\rho$ initial vector field $\mathscr{L}_{\rho}$ is expressible as some combinations, with constant coefficients, of standard fields $\frac{\partial}{\partial u_{j}}$ with $\left[u_{j}\right]=\left[w_{j}\right]=\rho$. Similar fact holds also for the above bracket $\left[\mathscr{L}_{\alpha, i}, \mathscr{L}_{\beta, j}\right]$. According to Theorem 1.1, the number of standard fields $\frac{\partial}{\partial u_{j}}$, with $\left[u_{j}\right]=\rho$ is exactly equal to the number of (linearly independent) initial vector fields of the length $\rho$. This implies that the $\mathbb{C}$-vector space generated by all standard fields $\frac{\partial}{\partial u_{j}}$ with $\left[u_{j}\right]=\rho$ is equal to that generated by the length $\rho$ initial vector fields $\mathscr{L}_{\rho}$. Consequently, as an element of this space, the Lie bracket $\left[\mathscr{L}_{\alpha, i}, \mathscr{L}_{\beta, j}\right]$ can be expressed as a linear combination of the length $\rho$ initial vector fields $\mathscr{L}_{\rho}$ with constant coefficients.

To continue the proof, now let $\ell=\alpha+\beta>\rho$ and suppose, to derive a contradiction, that $\left[\mathscr{L}_{\alpha, i}, \mathscr{L}_{\beta, j}\right] \not \equiv 0$. Then, according to Lemma 3.1, it should be a weight $-\ell$ vector field with polynomial coefficients:

$$
\left[\mathscr{L}_{\alpha, i}, \mathscr{L}_{\beta, j}\right]:=\sum_{i} \varphi_{i}(z, \bar{z}, w, \bar{w}) \frac{\partial}{\partial w_{i}}+\sum_{i} \psi_{i}(z, \bar{z}, w, \bar{w}) \frac{\partial}{\partial \bar{w}_{i}},
$$

where, consequently, each polynomial $\varphi_{i}$ and $\psi_{i}$ is of the weight $\left[w_{i}\right]-\ell$. Since these coefficients are of the polynomial type, then their weights are nonnegative and thus $\left[w_{i}\right] \geq$ $\ell>\rho$. This is a contradiction to the fact that among our coordinates there is no any complex variable of the weight absolutely bigger than $\rho$. 
Remark 3.3 According to the notations introduced before Definition 1.1, the complexified tangent bundle $\mathbb{C} \otimes T M_{k}$ of an arbitrary length $\rho \mathrm{CR}$ model $M_{k}$ of codimension $k$, admits the filtration:

$$
T^{1,0} M_{k}+T^{0,1} M_{k}=D_{1} \subset D_{2} \subset \cdots \subset D_{\rho}=\mathbb{C} \otimes T M_{k},
$$

where each $D_{\ell}$ is a subdistribution constituted by initial vector fields $\mathscr{L}_{l}$ of lengths $l \leqslant \ell$. The above proposition indicates that at each point $p \in M_{k}$ near the origin, $\mathbb{C} \otimes T_{p} M_{k}$ can be identified with the graded complex nilpotent Lie algebra:

$$
\mathfrak{m}:=\mathfrak{m}_{-\rho} \oplus \mathfrak{m}_{-\rho+1} \oplus \cdots \oplus \mathfrak{m}_{-1}
$$

where $\mathfrak{m}_{-1}:=D_{1}$ and where $\mathfrak{m}_{-\ell}:=D_{\ell} / D_{\ell-1}, \ell=2, \ldots, \rho$ is the $\mathbb{C}$-vector space generated by all initial vector fields of the precise weight $-\ell$. In this case, $D_{1}$ is called a distribution of constant type $\mathfrak{m}$.

\subsection{The associated initial coframes and their Darboux-Cartan structures}

For $\ell=1, \ldots, \rho$ and $i=1, \ldots, 2+k$, let us denote by $\sigma_{\ell, i}$ the dual CR 1 -form associated with the initial vector field $\mathscr{L}_{\ell, i}$. Since the collection of the weighted homogeneous vector fields $\left\{\mathscr{L}_{1,1}, \ldots, \mathscr{L}_{\rho, 2+k}\right\}$ forms a frame for the complexified bundle $\mathbb{C} \otimes T M_{k}$, then its dual set $\left\{\sigma_{1,1}, \ldots, \sigma_{\rho, 2+k}\right\}$ is a coframe for it.

Lemma 3.4 Given a frame $\left\{\mathscr{V}_{1}, \ldots, \mathscr{V}_{n}\right\}$ on an open subset of $\mathbb{R}^{n}$ enjoying the Lie structure:

$$
\left[\mathscr{V}_{i_{1}}, \mathscr{V}_{i_{2}}\right]=\sum_{k=1}^{n} c_{i_{1}, i_{2}}^{k} \mathscr{V}_{k} \quad\left(1 \leqslant i_{1}<i_{2} \leqslant n\right),
$$

where the $c_{i_{1}, i_{2}}^{k}$ are certain functions on $\mathbb{R}^{n}$, the dual coframe $\left\{\omega^{1}, \ldots, \omega^{n}\right\}$ satisfying by definition:

$$
\omega^{k}\left(\mathscr{Y}_{i}\right)=\delta_{i}^{k}
$$

enjoys a quite similar Darboux-Cartan structure, up to an overall minus sign:

$$
d \omega^{k}=-\sum_{1 \leqslant i_{1}<i_{2} \leqslant n} c_{i_{1}, i_{2}}^{k} \omega^{i_{1}} \wedge \omega^{i_{2}} \quad(k=1 \ldots n) .
$$

As a direct consequence of the above Lemma and Proposition 3.2, we find the DarbouxCartan structure of our initial coframe as follows;

Proposition 3.5 The exterior differentiation of each 1-form $\sigma_{\ell}$ dual to the weight $-\ell$ initial vector field $\mathscr{L}_{\ell}$ is of the form:

$$
d \sigma_{\ell}:=\sum_{\beta+\gamma=\ell} \mathrm{c}_{\beta, \gamma} \sigma_{\beta} \wedge \sigma_{\gamma},
$$

for some constant complex integers $\mathrm{c}_{\beta, \gamma}$. This equivalently means that in the expression of each corresponding Lie bracket $\left[\mathscr{L}_{\beta}, \mathscr{L}_{\gamma}\right]$, with $\beta+\gamma=\ell$, the coefficient of $\mathscr{L}_{\ell}$ is $-\mathrm{c}_{\beta, \gamma}$.

Weight assignment Naturally, we assign the weight $-\ell$ to a certain 1 -form $\sigma_{\ell, i}$ and its differentiation $d \sigma_{\ell, i}$ as is the weight of their corresponding field $\mathscr{L}_{\ell, i}$. Also, we occasionally say that $\sigma_{\ell, i}$ is of the length $\ell$.

Another simple but quite useful result is as follows; 
Lemma 3.6 For each weight $-\ell$ initial 1 -form $\sigma_{\ell, i}$ with $\ell \neq 1$, there is a weight $-(\ell-1)$ initial 1-form $\sigma_{\ell-1, j}$ where either $\sigma_{\ell-1, j} \wedge \sigma_{1,1}$ or $\sigma_{\ell-1, j} \wedge \sigma_{1,2}$ is visible uniquely in the Darboux-Cartan structure of $d \sigma_{\ell, i}$.

Proof This is a straightforward consequence of the fact that in the procedure of constructing our initial frame, each weight $-\ell$ vector field $\mathscr{L}_{\ell, i}$ is constructed as the Lie bracket between $\mathscr{L}_{1,1}$ or $\mathscr{L}_{1,2}$ and a unique weight $-(\ell-1)$ vector filed $\mathscr{L}_{\ell-1, j}$. Then, Lemma 3.4 implies the desired results.

\subsection{Ambiguity matrix}

After providing the above appropriate initial frame and coframe on the complexified tangent bundle $\mathbb{C} \otimes T M_{k}$, now this is the time of seeking the ambiguity matrix associated with the problem, what actually encodes biholomorphic equivalences to $M_{k}$. The procedure of construction is demonstrated in the recent works $[21,24,29,32]$ in the specific cases of $k=$ $1,2,3,4$. Let us explain it here in the general case of the CR model $M_{k}$. Assume that:

$$
\begin{aligned}
h: M_{k} & \longrightarrow \mathbf{M}_{k} \\
(z, w) & \longmapsto\left(z^{\prime}(z, w), w^{\prime}(z, w)\right)
\end{aligned}
$$

is a (biholomorphic) equivalence map between our $(2+k)$-dimensional CR model $M_{k}$ and another arbitrary real analytic totally nondegenerate $\mathrm{CR}$ generic submanifold $\mathbf{M}_{k} \subset \mathbb{C}^{1+k}$ of codimension $k$, in canonical coordinates $\left(z^{\prime}, w_{1}^{\prime}, \ldots, w_{k}^{\prime}\right)$. We assume that $\mathbf{M}_{k}$ is also equipped with a frame of $2+k$ lifted vector fields $\left\{\mathbf{L}_{1,1}, \mathbf{L}_{1,2}, \mathbf{L}_{2,3}, \mathbf{L}_{3,4}, \mathbf{L}_{3,5}, \ldots, \mathbf{L}_{\rho, 2+k}\right\}$ where, as before, $\mathbf{L}_{1,1}$ and $\mathbf{L}_{1,2}=\overline{\mathbf{L}_{1,1}}$ are local generators of $T^{1,0} \mathbf{M}_{k}$ and $T^{0,1} \mathbf{M}_{k}$ and where each other vector field $\mathbf{L}_{\ell, i}$ can be computed as an iterated Lie bracket between $\mathbf{L}_{1,1}$ and $\mathbf{L}_{1,2}$ of the length $\ell$, exactly as (12) for constructing the initial vector filed $\mathscr{L}_{\ell, i}$. Tensoring with $\mathbb{C}$, then the push-forward $h_{*}: T M_{k} \longrightarrow T \mathbf{M}_{k}$ of $h$ induces a complexified map, still denoted by the same symbol with the customary abuse of notation [8]:

$$
\begin{aligned}
h_{*}: & \mathbb{C} \otimes T M_{k} \longrightarrow \mathbb{C} \otimes T \mathbf{M}_{k}, \\
& \mathrm{z} \otimes \mathscr{X} \longmapsto \mathrm{z} \otimes h_{*}(\mathscr{X}) .
\end{aligned}
$$

Our current purpose is to seek the associated matrix to this linear map.

According to principles in CR geometry $[1,8,19], h_{*}$ transfers every generator of $T^{1,0} M_{k}$ to a vector field in the same bundle $T^{1,0} \mathbf{M}_{k}$. Hence for the single generator $\mathscr{L}_{1,1}$ of $T^{1,0} M_{k}$, there exists some nonzero function $a_{1}:=a_{1}\left(z^{\prime}, w^{\prime}\right)$ with:

$$
h_{*}\left(\mathscr{L}_{1,1}\right)=a_{1} \mathbf{L}_{1,1} .
$$

Moreover, $h_{*}$ preserves the conjugation, whence for $\mathscr{L}_{1,2}:=\overline{\mathscr{L}_{1,1}}$, we have $h_{*}\left(\mathscr{L}_{1,2}\right)=$ $\bar{a}_{1} \mathbf{L}_{1,2}$.

The third vector field in the basis of $\mathbb{C} \otimes T M_{k}$ is the imaginary field $\mathscr{L}_{2,1}:=\left[\mathscr{L}_{1,1}, \mathscr{L}_{1,2}\right]$. Let us compute the image of $h_{*}$ on it:

$$
\begin{aligned}
h_{*}\left(\mathscr{L}_{2,3}\right) & =h_{*}\left(\left[\mathscr{L}_{1,1}, \mathscr{L}_{1,2}\right]\right)=\left[h_{*}\left(\mathscr{L}_{1,1}\right), h_{*}\left(\mathscr{L}_{1,2}\right)\right]=\left[a_{1} \mathbf{L}_{1,1}, \bar{a}_{1} \mathbf{L}_{1,2}\right] \\
& =a_{1} \bar{a}_{1}\left[\mathbf{L}_{1,1} \mathbf{L}_{1,2}\right] \underbrace{-\bar{a}_{1} \mathbf{L}_{1,2}\left(a_{1}\right)}_{=: a_{2}} \mathbf{L}_{1,1}+a_{1} \mathbf{L}_{1,1}\left(\bar{a}_{1}\right) \mathbf{L}_{1,2} \\
& =: a_{1} \bar{a}_{1} \mathbf{L}_{2,3}+a_{2} \mathbf{L}_{1,1}-\bar{a}_{2} \mathbf{L}_{1,2},
\end{aligned}
$$

for a certain function $a_{2}:=a_{2}\left(z^{\prime}, w^{\prime}\right)$. 
Next, in the length three, two initial fields $\mathscr{L}_{3,4}=\left[\mathscr{L}_{1,1}, \mathscr{L}_{2,3}\right]$ and $\mathscr{L}_{3,5}=-\left[\mathscr{L}_{1,2}, \mathscr{L}_{2,3}\right]$ exist. Without affecting the results, we multiply the second bracket by -1 to have the simple relation $\mathscr{L}_{3,5}=\overline{\mathscr{L}_{3,4}}$. In a similar fashion of computations, one finds:

$$
\begin{aligned}
h_{*}\left(\mathscr{L}_{3,4}\right):= & a_{1}^{2} \bar{a}_{1} \mathbf{L}_{3,4}+\underbrace{\left(a_{1} \mathbf{L}_{1,1}\left(a_{1} \bar{a}_{1}\right)-a_{1} \bar{a}_{2}\right)}_{=: a_{3}} \mathbf{L}_{2,3}+ \\
& +\underbrace{\left(-a_{1} \bar{a}_{1} \mathbf{L}_{2,3}\left(a_{1}\right)+a_{1} \mathbf{L}_{1,1}\left(a_{2}\right)-a_{2} \mathbf{L}_{1,1}\left(a_{1}\right)+\bar{a}_{2} \mathbf{L}_{1,2}\left(a_{1}\right)\right)}_{=: a_{4}} \mathbf{L}_{1,1} \underbrace{-a_{1} \mathbf{L}_{1,1}\left(\bar{a}_{2}\right)}_{=: a_{5}} \mathbf{L}_{1,2},
\end{aligned}
$$

for some three certain complex functions $a_{j}:=a_{j}\left(z^{\prime}, w^{\prime}\right), j=3,4,5$. By conjugation, we also have:

$$
h_{*}\left(\mathscr{L}_{3,5}\right)=a_{1} \bar{a}_{1}^{2} \mathbf{L}_{3,5}-\bar{a}_{3} \mathbf{L}_{2,3}+\bar{a}_{5} \mathbf{L}_{1,1}+\bar{a}_{4} \mathbf{L}_{1,2} .
$$

Proceeding along the same lines of computations and by an induction on the weight of the initial fields, one finds the following general expression for the image of the complexified push-forward map $h_{*}$;

Lemma 3.7 For a fixed length $\ell$ initial vector field $\mathscr{L}_{\ell, i}$, the push-forward map $h_{*}$ transfers it to a combination like:

$$
h_{*}\left(\mathscr{L}_{\ell, i}\right):=a_{1}^{p_{i}} \bar{a}_{1}^{q_{i}} \mathbf{L}_{\ell, i}+\sum_{l<\ell} \mathrm{a}_{r_{j}} \mathbf{L}_{l, r}, \quad \text { with } \quad p_{i}+q_{i}=\ell
$$

where $\mathrm{a}_{r_{j}}$ s are some (possibly zero) complex functions in terms of the target coordinates $\left(z^{\prime}, w^{\prime}\right)$. In other words, $h_{*}\left(\mathscr{L}_{\ell, i}\right)$ is a combination of the corresponding lifted vector field $\mathbf{L}_{\ell, i}$ and some other ones $\mathbf{L}_{l, r}$ of absolutely smaller lengths $l<\ell$. Moreover, two integers $p_{i}$ and $q_{i}$ are actually the numbers of appearing $\mathscr{L}_{1,1}$ and $\mathscr{L}_{1,2}$ in the construction of $\mathscr{L}_{\ell, i}$ as an iterated bracket of them.

Then, our sought invertible matrix associated with $h_{*}$ is a $(2+k) \times(2+k)$ upper triangular matrix satisfying - here we drop the push-forward $h_{*}$ at the left hand side, for simplicity:

$$
\left(\begin{array}{c}
\mathscr{L}_{\rho, i} \\
\mathscr{L}_{\rho-1, j} \\
\vdots \\
\mathscr{L}_{3,5} \\
\mathscr{L}_{3,4} \\
\mathscr{L}_{2,3} \\
\mathscr{L}_{1,2} \\
\mathscr{L}_{1,1}
\end{array}\right)=\left(\begin{array}{cccccccc}
a_{1}^{p_{i}} \bar{a}_{1}^{q_{i}} & \mathrm{a}_{\bullet} & \mathrm{a}_{\bullet} & \mathrm{a}_{\bullet} & \mathrm{a}_{\bullet} & \mathrm{a}_{\bullet} & \mathrm{a}_{\bullet} & \mathrm{a}_{\bullet} \\
0 & a_{1}^{p_{j}} \bar{a}_{1}^{q_{j}} & \mathrm{a}_{\bullet} & \mathrm{a}_{\bullet} & \mathrm{a}_{\bullet} & \mathrm{a}_{\bullet} & \mathrm{a}_{\bullet} & \mathrm{a}_{\bullet} \\
0 & 0 & \ddots & \mathrm{a}_{\bullet} & \ldots & \ldots & \ldots & \mathrm{a}_{\bullet} \\
0 & \ldots & 0 & a_{1} \bar{a}_{1}^{2} & 0 & -\bar{a}_{3} & \bar{a}_{4} & \bar{a}_{5} \\
0 & \ldots & 0 & 0 & a_{1}^{2} \bar{a}_{1} & a_{3} & a_{5} & a_{4} \\
0 & 0 & \ldots & \ldots & 0 & a_{1} \bar{a}_{1} & -\bar{a}_{2} & a_{2} \\
0 & 0 & 0 & \ldots & \ldots & 0 & \bar{a}_{1} & 0 \\
0 & 0 & 0 & 0 & \ldots & \ldots & 0 & a_{1}
\end{array}\right) \cdot\left(\begin{array}{c}
\mathbf{L}_{\rho, i} \\
\mathbf{L}_{\rho-1, j} \\
\vdots \\
\mathbf{L}_{3,5} \\
\mathbf{L}_{3,4} \\
\mathbf{L}_{2,3} \\
\mathbf{L}_{1,2} \\
\mathbf{L}_{1,1}
\end{array}\right) .
$$

If on the main diagonal of the matrix and in front of $\mathscr{L}_{\ell, r}$ we have $a^{p_{r}} \bar{a}^{q_{r}}$, then $p_{r}+q_{r}=\ell$. As a result of explicitness in the already procedure of constructing the above desired matrix, we have also the following key observation;

Lemma 3.8 In the case that both the specific group parameters $a_{2}$ and $a_{3}$, appeared in (14) and (15), vanish then all the next parameters $a_{4}, a_{5}, \ldots$ vanish, identically.

Proof First we claim that all the appearing group parameters $a_{j}$ with $j>1$ are some combinations of the iterated $\left\{\mathbf{L}_{1,1}, \mathbf{L}_{1,2}\right\}$-differentiations of the first parameter $a_{1}$ and its conjugation $\bar{a}_{1}$. We prove our claim by an induction on the length of the initial fields. By (14), the claim holds for $a_{2}$ and as our induction hypothesis, assume that it holds for all group 
parameters $a_{\bullet}$ appearing among computing the image of $h_{*}$ on initial fields $\mathscr{L}_{l}$ of the lengths $\leqslant \ell$. Then for an initial field $\mathscr{L}_{\ell+1}=\left[\mathscr{L}_{1,1}, \mathscr{L}_{\ell, i}\right]$ of the next length $\ell+1$ (similar argument holds if we have $\mathscr{L}_{1,2}$ in place of $\mathscr{L}_{1,1}$ ) and according to Lemma 3.7 we have:

$$
h_{*}\left(\mathscr{L}_{\ell+1}\right)=\left[h_{*}\left(\mathscr{L}_{1,1}\right), h_{*}\left(\mathscr{L}_{\ell, i}\right)\right]=\left[a_{1} \mathbf{L}_{1,1}, a_{1}^{p} \bar{a}_{1}^{q} \mathbf{L}_{\ell, i}+\sum_{l<\ell} \mathrm{a}_{r_{j}} \mathbf{L}_{l, r}\right],
$$

where, by hypothesis induction, the appearing coefficients $\mathrm{a}_{r_{j}}$ are some combinations of the iterated $\left\{\mathbf{L}_{1,1}, \mathbf{L}_{1,2}\right\}$-differentiations of $a_{1}$ and $\bar{a}_{1}$. Computing this bracket by means of the Leibniz rule, one finds the new coefficients, namely new group parameters, again as some combinations of the iterated $\left\{\mathbf{L}_{1,1}, \mathbf{L}_{1,2}\right\}$-differentiations of $a_{1}$ and $\bar{a}_{1}$. This completes the proof of our claim.

Now, according to (14) and (15) we have:

$$
a_{2}=-\bar{a}_{1} \mathbf{L}_{1,2}\left(a_{1}\right) \text { and } a_{3}=a_{1} \mathbf{L}_{1,1}\left(a_{1} \bar{a}_{1}\right)-a_{1} \bar{a}_{2} .
$$

Since $a_{1} \neq 0$, then vanishing of $a_{2}$ and $a_{3}$ implies that-reminding $\mathbf{L}_{1,2}=\overline{\mathbf{L}_{1,1}}$ :

$$
\mathbf{L}_{1,1}\left(\bar{a}_{1}\right) \equiv 0, \quad \mathbf{L}_{1,1}\left(a_{1}\right) \equiv 0, \quad \mathbf{L}_{1,2}\left(a_{1}\right) \equiv 0, \quad \mathbf{L}_{1,2}\left(\bar{a}_{1}\right) \equiv 0 .
$$

Thus according to our claim, if $a_{2}$ and $a_{3}$ vanish then all the next group parameters $a_{j}$ vanish, identically.

Weight assignment Let $a_{j}$ be a group parameter which is appeared among computing the value of $h_{*}$ on a length $\ell$ initial vector field $\mathscr{L}_{\ell}$. Then, we assign the weight $\ell$ to this group parameter and its conjugation $\bar{a}_{j}$. For example, according to (13), (14) and (15) we have:

$$
\left[a_{1}\right]=1, \quad\left[a_{2}\right]=2, \quad\left[a_{3}\right]=\left[a_{4}\right]=\left[a_{5}\right]=3 .
$$

By this assignment, the nonzero entries at each row of the above matrix (17) have equal weight.

For each lifted vector field $\mathbf{L}_{\ell, i}$, let us denote by $\Gamma_{\ell, i}$ its dual lifted 1 -form and as its corresponding initial 1 -form $\sigma_{\ell, i}$, assign the weight $-\ell$ to it. The sough ambiguity matrix $\mathbf{g}$ of our equivalence problem, in question, is defined as the invertible matrix associated with the dual pull-back $h^{*}: \mathbb{C} \otimes T^{*} \mathbf{M}_{k} \rightarrow \mathbb{C} \otimes T^{*} M_{k}$ of the push-forward $h_{*}$. Then, after a plain matrix transposition we have:

$$
\left(\begin{array}{c}
\Gamma_{\rho, i} \\
\vdots \\
\Gamma_{\rho-1, j} \\
\vdots \\
\Gamma_{3,5} \\
\Gamma_{3,4} \\
\Gamma_{2,3} \\
\Gamma_{1,2} \\
\Gamma_{1,1}
\end{array}\right)=\underbrace{\left(\begin{array}{cccccccc}
a_{1}^{p_{i}} \bar{a}_{1}^{q_{i}} & 0 & 0 & 0 & 0 & 0 & 0 & 0 \\
\mathbf{0} & \vdots & \vdots & \vdots & \vdots & \vdots & \vdots & \vdots \\
\mathrm{a}_{\bullet} & a_{1}^{p_{j}} \bar{a}_{1}^{q_{j}} & 0 & 0 & \ldots & \ldots & 0 & 0 \\
\mathrm{a}_{\bullet} & \mathrm{a}_{\bullet} & \ddots & 0 & 0 & \ldots & \ldots & 0 \\
\mathrm{a}_{\bullet} & \mathrm{a}_{\bullet} & \ldots & a_{1} \bar{a}_{1}^{2} & 0 & 0 & \ldots & 0 \\
\mathrm{a}_{\bullet} & \ldots & \ldots & 0 & a_{1}^{2} \bar{a}_{1} & 0 & \ldots & 0 \\
\mathrm{a}_{\bullet} & \ldots & \ldots & -\bar{a}_{3} & a_{3} & a_{1} \bar{a}_{1} & 0 & 0 \\
\mathrm{a}_{\bullet} & \ldots & \ldots & \bar{a}_{4} & a_{5} & -\bar{a}_{2} & \bar{a}_{1} & 0 \\
\mathrm{a}_{\bullet} & \mathrm{a}_{\bullet} & \ldots & \bar{a}_{5} & a_{4} & a_{2} & 0 & a_{1}
\end{array}\right)}_{\mathbf{g}}\left(\begin{array}{c}
\sigma_{\rho, i} \\
\vdots \\
\sigma_{\rho-1, j} \\
\vdots \\
\sigma_{3,5} \\
\sigma_{3,4} \\
\sigma_{2,3} \\
\sigma_{1,2} \\
\sigma_{1,1}
\end{array}\right) .
$$

Remark 3.9 Clarifying the structure of the matrix $\mathbf{g}$, it is important to notice that thanks to Lemma 3.7 and for each arbitrary $i$ th column of this matrix, the first nonzero entry, which stands at the diagonal, is of the form $a_{1}^{r} \bar{a}_{1}^{s}$. Even more, since the only length $\ell$ lifted vector field in the image $h_{*}\left(\mathscr{L}_{\ell, i}\right)$ in (16) is $\mathbf{L}_{\ell, i}$, we can state that: if the $i$ th row of the left (or right) 
hand side vertical matrix in (18) is of the weight $-\ell$, then all the entries at the $i$ th column of $\mathrm{g}$ standing below $a_{1}^{r} \bar{a}_{1}^{s}$ and in front of a weight $-\ell 1$-form $\Gamma_{\ell}$ are zero. This fact is shown for example by the zero vector $\mathbf{0}$ in the first column of $\mathbf{g}$ or by the entry 0 below $a_{1} \bar{a}_{1}^{2}$.

Lemma 3.10 If the 1-form at the $i$ th row of the left (or right) hand side vertical matrix of (18) is of the weight $-\ell$ then, all nonzero entries at the ith column of $\mathbf{g}$ are of the same weight $\ell$.

Proof It is a straightforward consequence of the two paragraphs mentioned before (18).

The collection of all invertible matrices of the form $\mathbf{g}$ constitutes a finite dimensional (matrix) Lie group $G$, called by the structure Lie group of the equivalence problem to the CR model $M_{k}$.

Recall that [see (9) and the paragraph after it] the defining equations of our $k$ codimensional $\mathrm{CR}$ model $M_{k} \subset \mathbb{C}^{1+k}$ are precisely those of a CR model $M_{k-1}$ of codimension $k-1$ added just by the last equation $w_{k}-\bar{w}_{k}=2 i \Phi_{k}(z, \bar{z}, w, \bar{w})$. Let us state a result that will be of use later;

Proposition 3.11 The $(1+k) \times(1+k)$ ambiguity matrix $\mathbf{g}_{k-1}$ associated with the CR model $M_{k-1}$ is a submatrix of the ambiguity matrix $\mathbf{g}$ associated with $M_{k}$, standing as [cf. (18)]:

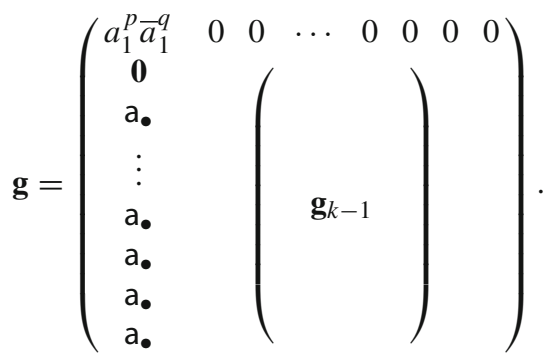

Proof Let $M_{k-1}$ be of the length $\rho^{\prime} \leq \rho$. If we proceed ab initio as Sect. 3.1 to provide an initial frame $\left\{\mathscr{L}_{1,1}^{\text {old }}, \mathscr{L}_{1,2}^{\text {old }}, \ldots, \mathscr{L}_{\rho^{\prime}, 1+k}^{\text {old }}\right\}$ for the $(1+k)$-dimensional CR model $M_{k-1}$, then according to its total nondegeneracy, one can construct the initial fields by means of the iterated Lie brackets of the generators $\mathscr{L}_{1,1}^{\text {old }}$ and $\mathscr{L}_{1,2}^{\text {old }}$ of $T^{1,0} M_{k-1}$ and $T^{0,1} M_{k-1}$; exactly as those for the initial vector fields on $M_{k}$ [cf. (12)] - here we assign the symbol "old" to objects corresponding to $M_{k-1}$. More precisely, if we have $\mathscr{L}_{\ell, j}=\left[\mathscr{L}_{1}, \mathscr{L}_{\ell-1, i}\right]$ for $j=1, \ldots, 1+k$, then correspondingly we have $\mathscr{L}_{\ell, j}^{\text {old }}=\left[\mathscr{L}_{1}^{\text {old }}, \mathscr{L}_{\ell-1, i}^{\text {old }}\right]$. Consequently, for a general biholomorphism $h^{\text {old }}: M_{k-1} \rightarrow \mathbf{M}_{k-1}$ and proceeding as Sect. 3.3 for the complexified push-forward $h_{*}^{\text {old }}: \mathbb{C} \otimes T M_{k-1} \rightarrow \mathbb{C} \otimes T \mathbf{M}_{k-1}$, one finds that if (cf. Lemma 3.7):

$$
h_{*}\left(\mathscr{L}_{\ell, j}\right):=a_{1}^{p} \bar{a}_{1}^{q} \mathbf{L}_{\ell, j}+\sum_{l<\ell} \mathrm{a}_{r_{i}} \mathbf{L}_{l, r} \quad(j=1, \ldots, 1+k),
$$

then correspondingly we also should have:

$$
h_{*}^{\text {old }}\left(\mathscr{L}_{\ell, j}^{\text {old }}\right):=a_{1}^{p} \bar{a}_{1}^{q} \mathbf{L}_{\ell, j}^{\text {old }}+\sum_{l<\ell} \mathrm{a}_{r_{i}} \mathbf{L}_{l, r}^{\text {old }} \quad(j=1, \ldots, 1+k),
$$

though in the former case the appearing group parameter-functions are in terms of the complex variables $z^{\prime}, w_{1}^{\prime}, \ldots, w_{k-1}^{\prime}, w_{k}^{\prime}$ and in the latter case they do not admit the last one $w_{k}^{\prime}$. The only distinction here is that the initial frame of $M_{k}$ has one more initial vector field, namely $\mathscr{L}_{\rho, 2+k}$ for which its image under $h_{*}$ should be computed, separately. This $h_{*}\left(\mathscr{L}_{\rho, 2+k}\right)$ manifests itself as the first column of $\mathbf{g}$. 
Remark 3.12 By an inspection of the above proof, one finds that among the construction of the ambiguity matrix associated with $M_{k-1}$, the assigned weights to all the appearing initial vector fields, 1 -forms and group parameters will be exactly as their corresponding items in the case of $M_{k}$.

\subsection{Associated structure equations}

According to our systematic strategy, introduced at the beginning of this section, now we are ready to compute the associated structure equations of the biholomorphic equivalence problem to the model $M_{k}$. Assuming $\Gamma:=\left(\Gamma_{\rho, 2+k}, \ldots, \Gamma_{1,1}\right)^{t}$ and $\Sigma:=\left(\sigma_{\rho, 2+k}, \ldots, \sigma_{1,1}\right)^{t}$ as our lifted and initial coframes, then by differentiating the both sides of the equality (18), which can be rewritten as $\Gamma=\mathbf{g} \cdot \Sigma$, gives:

$$
d \Gamma=d \mathbf{g} \wedge \Sigma+\mathbf{g} \cdot d \Sigma .
$$

For the first part $d \mathbf{g} \wedge \Sigma$ at the right hand side of this equation, one can replace it by:

$$
\underbrace{d \mathbf{g} \cdot \mathbf{g}^{-1}}_{\omega_{\mathrm{MC}}} \wedge \underbrace{\mathbf{g} \cdot \Sigma}_{\Gamma},
$$

where $\omega_{\mathrm{MC}}$ is the well-known Maurer-Cartan matrix of the Lie group $G$. Since $\mathbf{g}$ is lower triangular with the powers of the form $a_{1}^{r} \bar{a}_{1}^{s}$ on its main diagonal [cf. (18)], then $\omega_{\mathrm{MC}}$ is again lower triangular of the shape displaying in the following expanded form of the equation (20):

$$
\begin{aligned}
& \left(\begin{array}{c}
d \Gamma_{\rho, i} \\
d \Gamma_{\rho-1, j} \\
\vdots \\
d \Gamma_{3,5} \\
d \Gamma_{3,4} \\
d \Gamma_{2,3} \\
d \Gamma_{1,2} \\
d \Gamma_{1,1}
\end{array}\right)=\underbrace{\left(\begin{array}{ccccc}
p_{i} \alpha+q_{i} \bar{\alpha} & 0 & 0 & 0 & 0 \\
\delta_{\bullet} & p_{j} \alpha+q_{j} \bar{\alpha} & 0 & 0 & 0 \\
\vdots & \vdots & \ddots & \ldots & \ldots \\
\delta_{\bullet} & \delta_{\bullet} & \delta_{\bullet} & \bar{\alpha} & 0 \\
\delta_{\bullet} & \delta_{\bullet} & \delta_{\bullet} & \delta_{\bullet} & \alpha
\end{array}\right)}_{\omega_{\mathrm{MC}}} \wedge\left(\begin{array}{c}
\Gamma_{\rho, i} \\
\Gamma_{\rho-1, j} \\
\vdots \\
\Gamma_{3,5} \\
\Gamma_{3,4} \\
\Gamma_{2,3} \\
\Gamma_{1,2} \\
\Gamma_{1,1}
\end{array}\right) \\
& +\underbrace{\left(\begin{array}{cccccccc}
a_{1}^{p_{i}} \bar{a}_{1}^{q_{i}} & 0 & 0 & 0 & 0 & 0 & 0 & 0 \\
\mathrm{a}_{\bullet} & a_{1}^{p_{j}} \bar{a}_{1}^{q_{j}} & 0 & 0 & \ldots & \ldots & 0 & 0 \\
\mathrm{a}_{\bullet} & \mathrm{a}_{\bullet} & \ddots & 0 & 0 & \ldots & \ldots & 0 \\
\mathrm{a}_{\bullet} & \mathrm{a}_{\bullet} & \mathrm{a}_{\bullet} & a_{1} \bar{a}_{1}^{2} & 0 & 0 & \ldots & 0 \\
\mathrm{a}_{\bullet} & \vdots & \mathrm{a}_{\bullet} & 0 & a_{1}^{2} \bar{a}_{1} & 0 & \ldots & 0 \\
\mathrm{a}_{\bullet} & \vdots & \mathrm{a}_{\bullet} & -\bar{a}_{3} & a_{3} & a_{1} \bar{a}_{1} & 0 & 0 \\
\mathrm{a}_{\bullet} & \vdots & \mathrm{a}_{\bullet} & \bar{a}_{4} & a_{5} & -\bar{a}_{2} & \bar{a}_{1} & 0 \\
\mathrm{a}_{\bullet} & \mathrm{a}_{\bullet} & \mathrm{a}_{\bullet} & \bar{a}_{5} & a_{4} & a_{2} & 0 & a_{1}
\end{array}\right)} \cdot\left(\begin{array}{c}
d \sigma_{\rho, i} \\
d \sigma_{\rho-1, j} \\
\vdots \\
d \sigma_{3,5} \\
d \sigma_{3,4} \\
d \sigma_{2,3} \\
d \sigma_{1,2} \\
d \sigma_{1,1}
\end{array}\right),
\end{aligned}
$$

with $\alpha:=\frac{d a_{1}}{a_{1}}$ and with $\delta_{\bullet} \mathrm{s}$ as some (possibly zero) certain combinations of the standard forms

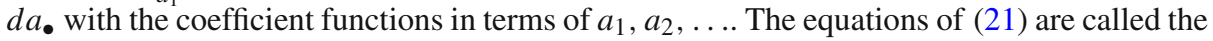
structure equations of the biholomorphic equivalence problem to $M_{k}$. The following lemma is encouraging enough to have some rigorous weight analysis on the structure equations in the next section. Recall that for each term $a_{j} d \sigma_{\ell, i}$, coming from the last matrix multiplication of (21), the associated weight is naturally defined as $\left[a_{j}\right]+\left[d \sigma_{\ell, i}\right]$. 
Lemma 3.13 All entries of the last vertical matrix $\mathbf{g} \cdot d \Sigma$ at the right hand side of the above structure equations (21) are homogeneous of the equal weight zero.

Proof It is a straightforward consequence of Lemma 3.10, reminding that the assigned weight to each $\sigma_{\ell}$ and its differentiation $d \sigma_{\ell}$ is $-\ell$.

\subsection{Torsion coefficients}

Our next aim is to restate the above structure equations (21) absolutely independent of the initial 1-forms $\sigma_{\ell, i}$ and their differentiations. For this purpose, we shall focus on the second matrix term $\mathbf{g} \cdot d \Sigma$. The Darboux-Cartan structure computed in Proposition 3.5 enables one to replace each 2-form $d \sigma_{\bullet}$ by some combination of the wedge products between initial 1-forms $\sigma_{\bullet}$. Afterward, by means of the equality $\Sigma=\mathbf{g}^{-1} \cdot \Gamma$, it is also possible to replace each initial 1 -form $\sigma_{\bullet}$ by some combination of the lifted 1 -forms $\Gamma_{\bullet}$. Doing so, then all differentiations at the right hand side vertical matrix $\mathbf{g} \cdot d \Sigma$ of (21) will be expressible in terms of the wedge products of the lifted 1 -forms $\Gamma_{.}$. Consequently, our structure equations will be converted into the form:

$$
\begin{aligned}
d \Gamma_{\ell, i}:= & \left(p_{i} \alpha+q_{i} \bar{\alpha}\right) \wedge \Gamma_{\ell, i}+\sum_{r, j, l \supsetneqq \ell} \delta_{r} \wedge \Gamma_{l, j} \\
& +\sum_{l, j, m, n} T_{j n}^{i}\left(a_{\bullet}\right) \Gamma_{l, j} \wedge \Gamma_{m, n}, \quad(\ell=1, \ldots, \rho, \quad i=1, \ldots, 2+k),
\end{aligned}
$$

where $T_{j n}^{i}$ s are some certain functions in terms of the group parameters $a_{\bullet}$ which are called by the torsion coefficients of the problem.

Remark 3.14 Since our ambiguity matrix $\mathbf{g}$ is invertible and lower triangular with the powers $a_{1}^{p} \bar{a}_{1}^{q}$ at its diagonal, then a simple induction on the number of its column and rows shows that $\mathbf{g}^{-1}$ is again lower triangular where its non-diagonal entries are some fraction polynomial functions with some powers of the form $a_{1}^{r} \bar{a}_{1}^{s}$ as their denominators. Also, if the $i$ th diagonal entry of $\mathbf{g}$ is, say, $a_{1}^{p} \bar{a}_{1}^{q}$ then this entry in $\mathbf{g}^{-1}$ is $\frac{1}{a_{1}^{p} \bar{a}_{1}^{q}}$. Finally, thanks to Lemma 3.7 and again since $\mathbf{g}$ is lower triangular, then in the expression of each length $\ell$ lifted 1 -from $\Gamma_{\ell, i}$ as (18), the only appearing initial 1 -form of the lengths $\leqslant \ell$ is $\sigma_{\ell, i}$. Consequently, by a backward induction on the length $\ell$ of the initial 1-forms from $\rho$ to 1 , we discover a same fact in expressing each initial 1 -form $\sigma_{\ell, i}$ in terms of the lifted ones through the equality $\Sigma=\mathbf{g}^{-1} \cdot \Gamma$ : the only appearing lifted 1 -form in the expression of $\sigma_{\ell, i}$ of the length $\leqslant \ell$ is $\Gamma_{\ell, i}$. Therefore, if the $j$ th row of the vertical matrix $\Sigma$, say $\sigma_{\ell, i}$, is of the length $\ell$ then the $j$ th row of $\mathbf{g}^{-1}$ is of the form:

$$
(c_{\bullet}, \ldots, c_{\bullet}, \underbrace{0, \ldots, 0}_{t_{1} \text { times }}, \underbrace{\frac{1}{a_{1}^{r} \bar{a}_{1}^{s}}}_{i \text { th place }}, \underbrace{0, \ldots, 0}_{t_{2} \text { times }})
$$

where $r+s=\ell$ and $t_{1}+t_{2}+1$ is equal or more than the number of initial 1-forms $\sigma_{\bullet}$ of the lengths $\leqslant \ell$. 


\section{Weight analysis on the structure equations}

In the previous section, we assigned naturally some weights to the complex variables, initial and lifted vector fields and 1-forms, their differentiations and also to group parameters. The main purpose of this section is to show that all the appearing torsion coefficients in the constructed structure equations (22) are weighted homogeneous of the same weight zero. For this aim, we inspect more the structure of the inverse matrix $\mathbf{g}^{-1}$ via some auxiliary lemmas. But at first we need the following definition.

Definition 4.1 Let:

$$
f\left(a_{1}, a_{2}, \ldots\right)=\frac{a_{1}^{r_{1}} \bar{a}_{1}^{s_{1}} a_{2}^{s_{2}} \bar{a}_{2}^{s_{2}} \ldots a_{n}^{r_{n}} \bar{a}_{n}^{s_{n}}}{a_{1}^{r} \bar{a}_{1}^{s}}
$$

be an arbitrary monomial fraction in terms of the group parameters. Then, the weight of $f$ is defined as:

$$
[f]=r_{1}\left[a_{1}\right]+s_{1}\left[\bar{a}_{1}\right]+r_{2}\left[a_{2}\right]+s_{2}\left[\bar{a}_{2}\right]+\cdots+r_{n}\left[a_{n}\right]+s_{n}\left[\bar{a}_{n}\right]-r\left[a_{1}\right]-s\left[\bar{a}_{1}\right] .
$$

A weighted homogeneous polynomial fraction is a sum of monomial fractions of the same weigh.

As stated in Lemma 3.10, all the nonzero entries in a fixed column of our ambiguity matrix $\mathbf{g}$ are of the same weight. Our next goal is to show that in the inverse matrix $\mathbf{g}^{-1}$, the rows enjoy a similar fact.

Lemma 4.2 Fix an integer $i_{0}=1, \ldots, 2+k$ and let $-\ell$ be the weight of a certain 1 -form $\sigma_{\ell}$ standing at the $i_{0}$ th row of the vertical matrix $\Sigma=\left(\sigma_{\rho, 2+k}, \ldots, \sigma_{1,2}, \sigma_{1,1}\right)^{t}$ in (18). Then,

(i) all the nonzero entries of the $i_{0}$ th row of $\mathbf{g}^{-1}$ are of the same homogeneous weight $-\ell$, too.

(ii) if the $j$ th row of $\Sigma$ is of the weight $-(\ell+1)$ and if the $\left(i_{0} j\right)$ th entry of $\mathbf{g}$ is $e_{i_{0} j}$ then, this entry in $\mathbf{g}^{-1}$ is of the form:

$$
-\frac{e_{i_{0} j}}{a_{1}^{m} \bar{a}_{1}^{n}}
$$

for some constant integers $m$ and $n$.

Proof We prove the both parts by an induction on the codimension $k$ of the models. The base of this induction is provided by inspecting the matrices introduced in [24, p. 89] for $k=2$ and [21, p.104] for $k=3$-according to the Conjecture 1.3 we are considering CR models of the lengths $\rho \geqslant 3$ which start from $k=2$. Assume that the assertions hold for all CR models of codimensions $<k-1$. By Proposition 3.11, if $\mathbf{g}_{k-1}$ is the ambiguity matrix of the equivalence problem to the CR model $M_{k-1}$, then:

$$
\mathbf{g}^{-1}=\left(\begin{array}{cccccc}
\frac{1}{a_{1}^{p} \bar{a}_{1}^{q}} & 0 & 0 & \cdots & 0 & 0 \\
\mathbf{0} & & & & \\
\mathbf{b}_{j} & & & \\
\vdots & & \mathbf{g}_{k-1}^{-1} & &
\end{array}\right),
$$

with $p+q=\rho$ and for some certain functions $\mathrm{b}_{\text {. }}$. Thus, according to our induction and by Remark 3.12, it suffices to prove (i) just for each entry $\mathrm{b}_{t}$ at some $i_{0}$ th row of $\mathbf{g}^{-1}$ with 
$i_{0} \neq 1$. According to Lemma 3.10, all the nonzero group parameters at the first column of $\mathbf{g}$ are of the same maximum weight $\rho$. By our induction hypothesis and except $\mathbf{b}_{t}$, we know that all the nonzero entries at the $i_{0}$ th row of $\mathbf{g}^{-1}$ are of the same weight $-\ell$. We show that if $\mathrm{b}_{t} \neq 0$, then it is of the same weight, too. Multiplying the $i_{0}$ th row of $\mathbf{g}^{-1}$ by the first column of $\mathbf{g}$ gives:

$$
\mathrm{b}_{t} \cdot\left(a_{1}^{p} \bar{a}_{1}^{q}\right)+\Psi=0
$$

where $\Psi$ is some function of the weight $\rho-\ell$. Taking into account that $p+q=\rho$, then the polynomial fraction $\mathrm{b}_{t}=-\frac{\Psi}{a_{1}^{p} \bar{a}_{1}^{q}}$ is of the homogeneous weight $\rho-\ell-\rho=-\ell$, as was expected.

For the second part (ii), and according to our induction, it suffices to prove it only for some of the entries $e_{i_{0}}$ at the first column of $\mathbf{g}$, namely for $j=1$. Since the first row of $\Sigma$ is of the weight $-\rho$, then we have to look for weight $-\ell=-(\rho-1)$ rows $i_{0}$ of the inverse matrix $\mathbf{g}^{-1}$. By the first part (i), these rows are in front of the weight $-(\rho-1)$ initial 1-forms $\sigma_{\rho-1, i}$ in the equation $\Sigma=\mathbf{g}^{-1} \cdot \Gamma$ and hence $e_{i_{0} 1}$ stands below the zero vector $\mathbf{0}$ at the first column of $\mathbf{g}$ [cf. (18)]. Hence, $i_{0} \neq 1$. Assume that $\mathrm{b}_{r}$ stands at the same entry of $\mathbf{g}^{-1}$ as $e_{i_{0}}$ in $\mathbf{g}$. We aim to show $\mathrm{b}_{r}=-\frac{e_{i_{0}} 1}{a_{1}^{m} \bar{a}_{1}^{n}}$. The $i_{0}$ th row of $\mathbf{g}^{-1}$ is of the form (cf. Remark 3.14):

$$
(\mathrm{b}_{r}, c_{1}, \ldots, c_{t}, 0, \ldots, 0, \underbrace{\frac{1}{a_{1}^{r} \bar{a}_{1}^{s}}}_{i_{0} \text { th place }}, 0, \ldots, 0),
$$

where $t+1$ is the number of the weight $-\rho$ lifted 1 -forms $\sigma_{\rho}$. Then, multiplying again the above $i_{0}$ th row of $\mathbf{g}^{-1}$ to the first column of $\mathbf{g}$ and granted the Remark 3.12 about the zero vector $\mathbf{0}$ at this column gives:

$$
(\mathrm{b}_{r}, c_{1}, \ldots, c_{t}, 0, \ldots, 0, \underbrace{\frac{1}{a_{1}^{r} \bar{a}_{1}^{s}}}_{i_{0} \text { th place }}, 0, \ldots, 0) \cdot(a_{1}^{p} \bar{a}_{1}^{q}, \underbrace{\mathbf{0}}_{t \text {-tuple }}, \ldots, \underbrace{e_{i_{0} 1}}_{i_{0} \text { th place }}, \ldots)^{t}=0 .
$$

Now, simplifying this equality after multiplication and solving it in terms of $\mathrm{b}_{r}$ gives $\mathrm{b}_{r}=$ $-\frac{e_{i_{0}} 1}{a_{1}^{p+r} \bar{a}_{1}^{q+s}}$, as desired.

Roughly speaking, the first part $(i)$ of this lemma states that for each fixed row of the three matrices appearing in the equation $\Sigma=\mathrm{g}^{-1} \cdot \Gamma$, all the nonzero entries are of the same negative weight. Furthermore, taking into account the shape of the lower triangular matrix $\mathbf{g}^{-1}$ and by the first part of the above lemma, one observes that (see also Remark 3.14);

Lemma 4.3 For each weight - $\ell$ initial 1 -form $\sigma_{\ell, i}$, its expression in terms of the lifted 1 -forms is as follows:

$$
\sigma_{\ell, i}:=\sum_{l \supsetneqq \ell} \mathrm{A}_{j}^{i}\left(a_{\bullet}\right) \Gamma_{l, j}+\frac{1}{a_{1}^{p_{i}} \bar{a}_{1}^{q_{i}}} \Gamma_{\ell, i},
$$

with $p_{i}+q_{i}=\ell$ and for some weighted homogeneous polynomial fractions $\mathrm{A}_{j}^{i}$ of the weight $-\ell$ where their denominators are some powers of only $a_{1}$ and $\bar{a}_{1}$. 
Now, we are ready to prove the main result of this section;

Proposition 4.4 All torsion coefficients $T_{j n}^{i}\left(a_{\bullet}\right)$ appearing among the structure equations (22) are weighted homogeneous polynomial fractions of the equal weight zero where their denominators are some powers of only $a_{1}$ and $\bar{a}_{1}$.

Proof According to (21), each structure equation can be expressed as:

$$
d \Gamma_{\ell, i}=\left(p_{i} \alpha+q_{i} \bar{\alpha}\right) \wedge \Gamma_{\ell, i}+\sum_{l \supsetneqq \ell} \delta_{i_{j}} \wedge \Gamma_{l, j}+\sum_{l \supsetneqq \ell} a_{i_{j}} d \sigma_{l, j}+a_{1}^{p_{i}} \bar{a}_{1}^{q_{i}} d \sigma_{\ell, i}
$$

with $p_{i}+q_{i}=\ell$. Our torsion coefficients come from the last parts:

$$
\sum_{l \supsetneqq \ell} a_{i_{j}} d \sigma_{l, j}+a_{1}^{p_{i}} \bar{a}_{1}^{q_{i}} d \sigma_{\ell, i}
$$

of this equation after replacing each differentiation $d \sigma_{\bullet}$ according to the Darboux-Cartan structure computed in Proposition 3.5 and next substituting each initial 1 -form $\sigma_{\bullet}$ with some combinations of lifted 1-forms $\Gamma$ • by means of the equality $\Sigma=\mathbf{g}^{-1} \cdot \Gamma$. Thanks to Lemma 3.13 , the weight of the coefficient $a_{i_{j}}$ in the term $a_{i_{j}} d \sigma_{l, j}$ of (24) is $l$. Moreover, according to Proposition 3.5 we have:

$$
d \sigma_{l, j}:=\sum_{\beta, \gamma} \mathrm{c}_{\beta, \gamma} \sigma_{\beta} \wedge \sigma_{\gamma} \text { with } \beta+\gamma=l
$$

After replacing the expressions of $\sigma_{\beta}$ and $\sigma_{\gamma}$ as Lemma 4.3, such Darboux-Cartan structure takes the form:

$$
a_{i_{j}} d \sigma_{l, j}=\sum_{l_{1}+l_{2} \geqslant l}\left(a_{i_{j}} \mathrm{~T}_{m, n}^{j}\left(a_{\bullet}\right)\right) \Gamma_{l_{1}, m} \wedge \Gamma_{l_{2}, n}
$$

where the polynomial fractions $\mathrm{T}_{m, n}^{j}$ are multiplications of some weight $-\beta$ and $-\gamma$ polynomial fractions with $\beta+\gamma=l$. Thus, all the coefficients $\mathrm{T}_{m, n}^{j}$ are of the same weight $-\beta-\gamma=-l$ and hence, each coefficient $a_{i j} \mathrm{~T}_{m, n}^{j}\left(a_{\bullet}\right)$ in the above expression is of the weight zero. Similar fact holds true also for the last term $a_{1}^{p_{i}} \bar{a}_{1}^{q_{i}} d \sigma_{\ell, i}$ of (24). Now, each torsion coefficient $T_{m, n}^{i}$ is made as the sum of coefficients of $\Gamma_{l_{1}, m} \wedge \Gamma_{l_{2}, n}$ in the expressions of all terms $a_{i j} d \sigma_{l, j}$ and $a_{1}^{p_{i}} \bar{a}_{1}^{q_{i}} d \sigma_{\ell, i}$, visible in (24). Therefore, it is of the weight zero, as claimed. The second part of the assertion is a consequence of Remark 3.14.

Before concluding this section, let us present another result of the second part (ii) of Lemma 4.2;

Lemma 4.5 If in the structure equation $d \Gamma_{\ell-1, m}$ of (21) we have the term $a_{j} d \sigma_{\ell, n}$ for some (possibly zero) group parameter $a_{j}$, then the coefficient of $\Gamma_{\ell, n}$ in the expression of $\sigma_{\ell-1, m}$, through the equation $\Sigma=\mathbf{g}^{-1} \cdot \Gamma$, is of the form $-\frac{a_{j}}{a_{1}^{r} \bar{a}_{1}^{s}}$ for some constant integers $r$ and $s$.

Proof The term $a_{j} d \sigma_{\ell, n}$ in (21) comes only from the second part $\mathbf{g} \cdot d \Sigma$ and hence the appearance of this term in the structure equation $d \Gamma_{\ell-1, m}$ means that the coefficient of $\sigma_{\ell, n}$ 
in the expression of $\Gamma_{\ell-1, m}$ - coming from the equality $\Gamma=\mathbf{g} \cdot \Sigma$ —is $a_{j}$ :

$$
\underbrace{\left(\begin{array}{c}
\vdots \\
\Gamma_{\ell-1, m} \\
\vdots
\end{array}\right)}_{\Gamma}=\underbrace{\left(\begin{array}{cccc}
\vdots & \vdots & \vdots & \vdots \\
\vdots & \vdots & \vdots & \vdots \\
\ldots & a_{j} & \ldots & \cdots \\
\vdots & \vdots & \vdots & \vdots
\end{array}\right)}_{\mathbf{g}} \cdot \underbrace{\left(\begin{array}{c}
\vdots \\
\sigma_{\ell, n} \\
\vdots \\
\vdots
\end{array}\right)}_{\Sigma} .
$$

By the above matrix equation and according to the second part (ii) of Lemma 4.2, we will have some $-\frac{a_{j}}{a_{1}^{r} \bar{a}_{1}^{s}}$ in $\mathbf{g}^{-1}$ in place of the same entry $a_{j}$ in $\mathbf{g}$. But this entry in the inverse matrix determines, through the equality $\Sigma=\mathbf{g}^{-1} \cdot \Gamma$, the coefficient of the lifted 1-form $\Gamma_{\ell, n}$ in the expression of $\sigma_{\ell-1, m}$.

This suggests that if we are seeking the coefficient of $\Gamma_{\ell, n}$ in the expression of some $\sigma_{\ell-1, m}$, then it is opposite to the fraction of the coefficient of $d \sigma_{\ell, n}$ in the structure equation $d \Gamma_{\ell-1, m}$ by some powers of $a_{1}$ and $\bar{a}_{1}$. This result will be of much use in the next section.

\section{Picking up an appropriate weighted homogeneous subsystem}

Now we are ready to apply Cartan's method on the biholomorphic equivalence problem of the CR model $M_{k}$. The first two essential steps of this method are absorption and normalization, based on some fundamental results introduced in [21, Proposition 4.7] (see also [23]). According to these results, one is permitted to substitute as follows each Maurer-Cartan 1 -form $\alpha$ and $\delta_{j}$ in the structure equations (22):

$$
\begin{gathered}
\alpha \mapsto \alpha+t_{2+k} \Gamma_{\rho, 2+k}+\cdots+t_{2} \Gamma_{1,2}+t_{1} \Gamma_{1,1}, \\
\delta_{j} \mapsto \delta_{j}+s_{2+k}^{j} \Gamma_{\rho, 2+k}+\cdots+s_{2}^{j} \Gamma_{1,2}+s_{1}^{j} \Gamma_{1,1},
\end{gathered}
$$

for arbitrary coefficient functions $t_{\bullet}$ and $s_{\bullet}^{\bullet}$. We can apply such substitutions and try to convert new (torsion) coefficients of the wedge products $\Gamma_{\ell_{1}, i_{1}} \wedge \Gamma_{\ell_{2}, i_{2}}$ to some constant integerspossibly zero-by appropriate determinations of the arbitrary functions $t_{\bullet}, s_{\bullet}^{\bullet}$ (this is the absorption step). For this purpose, it may be inadequate only such determination of these arbitrary functions but it necessitates also to determine-or normalize in this literaturesome of the group parameters, appropriately in terms of the other ones by equating to zero (or other constants) still remaining non-constant coefficients. These coefficients are called the essential torsion coefficients.

Thus to proceed along the absorption and normalization steps, one has to solve an arising polynomial system with $t_{\bullet}, s_{\bullet}^{\bullet}$ and some of the group parameters as its unknowns. The virtual importance of the solution of this system is not determining the coefficient functions $t_{\bullet}$ and $s_{\bullet}^{\bullet}$ but it is actually the found values of involving group parameters $a_{\bullet}$. Unfortunately, solving such arising polynomial system, specifically in this general manner, causes certainly some unavoidable and serious algebraic complexity. The main purpose of this section is to bypass and manipulate such complexity by picking up an appropriate and convenient subsystem that affords to bring all results we are seeking from the solution of the original system. Before explaining our practical method of constructing this desired susbsystem-which will be divided into two major parts - at first, we need the following auxiliary lemma; 
Lemma 5.1 Assume that $\sigma_{\ell-1, i} \wedge \sigma_{1, t}$, for $t=1$ or 2 , is the unique appearing wedge product in the Darboux-Cartan structure of $d \sigma_{\ell, j}$, as stated in Lemma 3.6. Then, among all the expressions of differentiations $d \sigma_{l, r}$, with $l \geqslant \ell$, in terms of the wedge products of the lifted 1-forms, a nonzero coefficient of $\Gamma_{\ell-1, i} \wedge \Gamma_{1, t}$ appears uniquely in $d \sigma_{\ell, j}$. Such coefficient is a fraction of the form $\frac{1}{a_{1}^{p} \bar{a}_{1}^{q}}$ for some constant integers $p$ and $q$.

Proof By Remark 3.14, in the expression of each $\sigma_{\ell^{\prime}, r}$ through the equality $\Sigma=\mathbf{g}^{-1} \cdot \Gamma$, the only appearing lifted 1 -form $\Gamma_{l, m}$ with $l \leqslant \ell^{\prime}$ is some $\frac{1}{a_{1}^{p} \bar{a}_{1}^{q}} \Gamma_{\ell^{\prime}, r}$. In particular, the only initial 1 -form having some coefficient of $\Gamma_{1,1}$ in its expression is $\sigma_{1,1}$ and this coefficient is $\frac{1}{a_{1}}$. Similarly, the only initial 1-form having some coefficient of $\Gamma_{1,2}$ is $\sigma_{1,2}$ with the coefficient $\frac{1}{\bar{a}_{1}}$. Consequently, in the expression of a fixed differentiation $d \sigma_{l_{0}, r}$ with $l_{0} \geqslant \ell$, one finds some nonzero coefficient of $\Gamma_{\ell-1, i} \wedge \Gamma_{1, t}$ whenever in its Darboux-Cartan structure, $d \sigma_{l_{0}, r}$ includes some nonzero coefficient of the wedge product $\sigma_{l^{\prime}, j} \wedge \sigma_{1, t}$ with $l^{\prime} \leqslant \ell-1$. We claim that $\sigma_{l^{\prime}, j}=\sigma_{\ell-1, i}$. Since $\sigma_{l^{\prime}, j} \wedge \sigma_{1, t}$ appears in the Darboux-Cartan structure of $d \sigma_{l_{0}, r}$ then Proposition 3.5 implies that $l^{\prime}+1=l_{0} \geqslant \ell$ and whence $l^{\prime} \geqslant \ell-1$. Consequently, $l^{\prime}=\ell-1$ and thus $\sigma_{l^{\prime}, j}=\sigma_{\ell-1, j}$. Furthermore, again by Remark 3.14, $\sigma_{\ell-1, i}$ is the only weight $-(\ell-1)$ initial form containing some nonzero coefficient of $\Gamma_{\ell-1, i}$ in its expression. This results that $\sigma_{\ell-1, j}=\sigma_{\ell-1, i}$, as was claimed. But on the other hand, according to our assumption, $\sigma_{\ell-1, i} \wedge \sigma_{1, t}$ appears uniquely in the Darboux-Cartan structure of $d \sigma_{\ell, j}$ and hence we should have $d \sigma_{l_{0}, r}=d \sigma_{\ell, j}$, as was desired. In addition, the coefficient $\Gamma_{\ell-1, i} \wedge \Gamma_{1, t}$ in $d \sigma_{\ell, j}$ comes from the wedge product $\sigma_{\ell-1, i} \wedge \sigma_{1, t}$ in its Darboux-Cartan structure and by what mentioned at the beginning of the proof, it will be nothing but some fraction $\frac{1}{a_{1}^{p} \bar{a}_{1}^{q}}$.

\subsection{Picking up an appropriate subsystem}

Our strategy of picking up appropriate torsion coefficients from the structure equations, after absorption, is divided into two essential parts depending upon the weight.

\subsubsection{First part: structure equations of the weights $-\ell=-1, \ldots,-(\rho-1)$}

Consider:

$$
\begin{aligned}
d \Gamma_{\ell, m}= & \left(p_{m} \alpha+q_{m} \bar{\alpha}\right) \wedge \Gamma_{\ell, m} \\
& +\sum_{l \supsetneqq \ell} \delta_{i_{t}} \wedge \Gamma_{l, j}+\sum_{l \geq \ell+2} a_{j_{n}} d \sigma_{l, n}+\sum_{r} a_{j_{r}} d \sigma_{\ell+1, r}+a_{1}^{p_{m}} \bar{a}_{1}^{q_{m}} d \sigma_{\ell, m},
\end{aligned}
$$

as a weight $-\ell$ structure equation in (21) for $\ell=1, \ldots, \rho-1$. We focus just on the terms $a_{j_{r}} d \sigma_{\ell+1, r}$ in the penultimate part $\sum_{r} a_{j_{r}} d \sigma_{\ell+1, r}$ of this structure equation. Lemma 3.13 implies that the group parameters $a_{j_{r}}$, visible in it, are of the weight $\ell+1$. As a consequence of Lemma 5.1 and in the expression of each fixed term $a_{j_{r_{0}}} d \sigma_{\ell+1, r_{0}}$, in terms of the wedge products of lifted 1-forms, one finds a certain product:

$$
\frac{a_{j_{r_{0}}}}{a_{1}^{p_{\bullet}} \bar{a}_{1}^{q_{\bullet}}} \Gamma_{\ell, i_{j}} \wedge \Gamma_{1, t_{r}} \quad\left(t_{r}=1 \text { or } 2\right),
$$

coming from some $\sigma_{\ell, i_{j}} \wedge \sigma_{1, t_{r}}$, uniquely appearing in the Darboux-Cartan structure of $d \sigma_{\ell+1, r_{0}}$, such that no any other term in the part $\sum_{l \geq \ell+2, n} a_{j_{n}} d \sigma_{l, n}+\sum_{r \neq r_{0}} a_{j_{r}} d \sigma_{\ell+1, r}$ of (26) brings any nonzero coefficient of it. Then, as is our strategy, we seek for all coefficients of this wedge product $\Gamma_{\ell, i_{j}} \wedge \Gamma_{1, t_{r}}$ in (26). Let us do it part by part. 
We continue with the last term $a_{1}^{p_{m}} \bar{a}_{1}^{q_{m}} d \sigma_{\ell, m}$. Assuming the Darboux-Cartan structure:

$$
d \sigma_{\ell, m}=\sum_{\ell_{1}+\ell_{2}=\ell} c_{\ell_{1}, \ell_{2}} \sigma_{\ell_{1}, t} \wedge \sigma_{\ell_{2}, s}
$$

then the desired wedge product $\Gamma_{\ell, i_{j}} \wedge \Gamma_{1, t_{r}}$ is producible only by the terms of the form 4 $c_{t} \sigma_{\ell-1, t} \wedge \sigma_{1, t_{r}}$. In order to find the coefficient of this product, we have to pick the coefficient of $\Gamma_{\ell, i_{j}}$ in the expression of $\sigma_{\ell-1, t}$ s. By Lemma 4.5 and if the coefficient of $d \sigma_{\ell, i_{j}}$ in the structure equation of $d \Gamma_{\ell-1, t}$ is a weight $\ell$ group parameter $a_{i_{t}}$, then the desired coefficient of $\Gamma_{\ell, i_{j}}$ in $\sigma_{\ell-1, t}$ will be of the form $-\frac{a_{i t}}{a_{1}^{r_{\bullet}^{\prime}} a_{1}^{s_{\bullet}^{\prime}}}$ for some constant integers $r_{\bullet}^{\prime}$ and $s_{\bullet}^{\prime 5}$. Hence the last term $a_{1}^{p_{m}} \bar{a}_{1}^{q_{m}} d \sigma_{\ell, m}$ may produce some term like:

$$
-\left(\sum_{t} c_{\mathrm{t}} \frac{a_{i_{t}}}{a_{1}^{r_{\bullet}} \bar{a}_{1}^{s_{\bullet}}}\right) \Gamma_{\ell, i_{j}} \wedge \Gamma_{1, t_{r}},
$$

after simplification. For later use, we emphasize from the above procedure that;

Lemma 5.2 For each weight $\ell$ group parameter $a_{i_{t}}$ appearing in (28), there is a term $a_{i_{t}} d \sigma_{\ell, i_{j}}$ in some weight $-(\ell-1)$ structure equations $d \Gamma_{\ell-1, t}$.

Obviously, the second term $\sum_{l \supsetneqq \ell} \delta_{i_{t}} \wedge \Gamma_{l, j}$ of (26) will not produce any nonzero coefficient of $\Gamma_{\ell, i_{j}} \wedge \Gamma_{1, t_{r}}$ while after the substitutions (25) in the first part $\left(p_{m} \alpha+q_{m} \bar{\alpha}\right) \wedge \Gamma_{\ell, m}$, one may find some terms like:

$$
-\left(p_{m} t_{1}+q_{m} \bar{t}_{2}\right) \Gamma_{\ell, m} \wedge \Gamma_{1,1} \text { and }-\left(p_{m} t_{2}+q_{m} \bar{t}_{1}\right) \Gamma_{\ell, m} \wedge \Gamma_{1,2},
$$

where in the case that $m=i_{j}$, one of them will be the sought product $\Gamma_{\ell, i_{j}} \wedge \Gamma_{1, t_{r}}$, in question.

Then, all possible coefficients of the wedge product $\Gamma_{\ell, i_{j}} \wedge \Gamma_{1, t_{r}}$ in the above weight $-\ell$ structure equation (26), after absorption, are those presented in (27)-(29). Equating this coefficient to zero-as is the method of absorption-normalization-then one finds some fraction polynomial equation of the form:

$$
\frac{a_{j_{r_{0}}}}{a_{1}^{p_{\bullet}} \bar{a}_{1}^{q_{\bullet}}}-\sum_{t} \mathrm{c}_{\mathrm{t}} \frac{a_{i_{t}}}{a_{1}^{r_{\bullet}} \bar{a}_{1}^{s_{\bullet}}}=\mathrm{a}_{j_{r_{0}}} t_{1}+\mathrm{b}_{j_{r_{0}}} t_{2}+\mathrm{a}_{j_{r_{0}}}^{\prime} \bar{t}_{1}+\mathrm{b}_{j_{r_{0}}}^{\prime} \bar{t}_{2},
$$

for some (possibly zero) constants $\mathrm{a}_{j_{r_{0}}}, \mathrm{~b}_{j_{r_{0}}}, \mathrm{a}_{j_{r_{0}}}^{\prime}, \mathrm{b}_{j_{r_{0}}}^{\prime}$. The left hand side of this equation is actually the torsion coefficient $T_{i_{j}, t_{r}}^{m}$ of $\Gamma_{\ell, i_{j}} \wedge \Gamma_{1, t_{r}}$ in the structure equation (26) which comes from (27) and (28). Hence according to Proposition 4.4, it is of the weight zero. Minding that here $a_{j_{r_{0}}}$ is a weight $\ell+1$ group parameter while $a_{i_{t}}$ s are of the weight $\ell$, then multiplying both side of this equation by the denominator $a_{1}^{p \bullet} \bar{a}_{1}^{q \bullet}$ gives the following equivalent weighted homogeneous polynomial equation - here we assign naturally the weight zero to the parameters $t_{1}, t_{2}$ and their conjugations:

$$
a_{j_{r_{0}}}-\sum_{t} \mathrm{c}_{t} a_{1}^{r_{\bullet}^{\prime}} \bar{a}_{1}^{s_{\bullet}^{\prime}} a_{i_{t}}=a_{1}^{p \bullet} \bar{a}_{1}^{q \bullet}\left(\mathrm{a}_{j_{r_{0}}} t_{1}+\mathrm{b}_{j_{r_{0}}} t_{2}+\mathrm{a}_{j_{r_{0}}}^{\prime} \bar{t}_{1}+\mathrm{b}_{j_{r_{0}}}^{\prime} \bar{t}_{2}\right) \text {. }
$$

\footnotetext{
4 Remind that one can find the lifted 1 -forms $\Gamma_{1,1}$ and $\Gamma_{1,2}$ only in the expressions of $\sigma_{1,1}$ and $\sigma_{1,2}$, respectively.

5 Notice also that here $a_{i_{t}}$ can be zero and it does not effect our next results.
} 
Proposition 5.3 Let $\ell=1, \ldots, \rho-1$. Then, among the procedure of absorption and associated with each weight $\ell+1$ group parameter $a_{j_{r_{0}}}$ appearing in an arbitrary weight $-\ell$ structure equation (26), one finds a weighted homogeneous parametric complex polynomial equation as (30), expressing $a_{j_{r_{0}}}$ in terms of $a_{1}, \bar{a}_{1}$, some weight $\ell$ group parameters $a_{i_{t}}$, two parameters $t_{1}, t_{2}$ and their conjugations.

Let us denote by $\mathrm{S}$ the weighted homogeneous system of equations mentioned in the above proposition. Notice that $S$ does not involve necessarily all the group parameters $a_{\bullet}$. Importantly in this system, if there is an equation like (30) that expresses a weight $\ell+1$ group parameter $a_{j_{r_{0}}}$ in terms of the weight $\ell$ parameters $a_{i_{t}}$, then Lemma 5.2 guarantees that also for each $a_{i_{t}}$, we find another equation expressing it in terms of the lower weight group parameters when we perform the above method for weight $-(\ell-1)$ structure equations.

Proposition 5.4 For each group parameter $a_{j} \neq a_{1}$ visible in $\mathrm{S}$, there exists some weighted homogeneous equation expressing it in terms of some lower weight group parameters $a_{\bullet}$ and the parameters $t_{1}, t_{2}$.

Among the system S, two equations coming from the first two structure equations:

$$
\begin{aligned}
& d \Gamma_{2,3}=(\alpha+\bar{\alpha}) \wedge \Gamma_{2,3}+\sum_{l \supsetneqq 2} \delta_{i_{j}} \wedge \Gamma_{l, j}+\sum_{l \supsetneqq 3} a_{i_{j}} d \sigma_{l, j}+a_{3} d \sigma_{3,4}+\bar{a}_{3} d \sigma_{3,5}+a_{1} \bar{a}_{1} d \sigma_{2,3}, \\
& d \Gamma_{1,1}=\alpha \wedge \Gamma_{1,1}+\sum_{l \supsetneqq 1} \delta_{i_{j}} \wedge \Gamma_{l, j}+\sum_{l \supsetneqq 2} a_{i_{j}} d \sigma_{l, j}+a_{2} d \sigma_{2,3}+a_{1} d \sigma_{1,1}
\end{aligned}
$$

are of particular importance. According to our suggested method, in the weight -2 structure equation $d \Gamma_{2,3}$ we should focus on the term $a_{3} d \sigma_{3,4}$ since $d \sigma_{3,4}$, together with $d \sigma_{3,5}$, are the only weight $-(2+1)=-3$ differentiations visible in it. Since $\mathscr{L}_{3,4}=\left[\mathscr{L}_{1,1}, \mathscr{L}_{2,3}\right]$, then the uniquely appearing wedge product in the Darboux-Cartan structure of $d \sigma_{3,4}$ is $\sigma_{2,3} \wedge \sigma_{1,1}$ (cf. Lemma 3.6 and its proof). Thus, we shall look for the (torsion) coefficient of $\Gamma_{2,3} \wedge \Gamma_{1,1}$ in this structure equation $d \Gamma_{2,3}$. Also in the weight -1 structure equation $d \Gamma_{1,1}$ we should focus on the single term $a_{2} d \sigma_{2,3}$. The uniquely appearing wedge product in the DarbouxCartan structure of $d \sigma_{2,3}$ is $\sigma_{1,2} \wedge \sigma_{1,1}$, then let us find the coefficient of $\Gamma_{1,2} \wedge \Gamma_{1,1}$ in this structure equation. Performing necessary computations, we, respectively, find the following two weight zero homogeneous equations, after applying the substitutions (25):

$$
\frac{a_{3}}{a_{1}^{2} \bar{a}_{1}}+i \frac{\bar{a}_{2}}{a_{1} \bar{a}_{1}}=t_{1}+\bar{t}_{2}, \quad i \frac{a_{2}}{a_{1} \bar{a}_{1}}=t_{2},
$$

which give, surprisingly, the parameters $t_{1}$ and $t_{2}$ as some weight zero expressions:

$$
t_{1}=\frac{a_{3}}{a_{1}^{2} \bar{a}_{1}}+2 i \frac{\bar{a}_{2}}{a_{1} \bar{a}_{1}}, \quad t_{2}=i \frac{a_{2}}{a_{1} \bar{a}_{1}} .
$$

Putting these expressions in $S$ and multiplying again the appearing fractional equations by some sufficient powers of $a_{1}$ and $\bar{a}_{1}$, then one finds $S$ as a weighted homogeneous polynomial system with no any parameter. Except $a_{2}$ and $a_{3}$ that we already spent their associated equations (32) to find the expressions of the parameters $t_{1}$ and $t_{2}$, for each other involving group parameters $a_{\bullet}$ there exists one equation in $S$ that expresses it in terms of some lower weight group parameters. Our next goal is to provide two more polynomial equations including $a_{2}$ and $a_{3}$ to recover this constraint. 


\subsubsection{Second part: structure equations of the weight $-\rho$}

One might be somehow surprised that so far we did not talk about the weight $-\rho$ structure equations. In fact, our trick was to retain them for our current aim of providing at least two more weighted homogeneous equations. ${ }^{6}$ Notice that the method suggested above, can not be applied on a weight $-\rho$ structure equation:

$$
d \Gamma_{\rho, i}=\left(p_{i} \alpha+q_{i} \bar{\alpha}\right) \wedge \Gamma_{\rho, i}+a_{1}^{p_{i}} \bar{a}_{1}^{q_{i}} d \sigma_{\rho, i}
$$

since it essentially does not contain any term $a_{\bullet} d \sigma_{\bullet}$ with $d \sigma_{\bullet}$ of the weight $-(\rho+1)$. However, here we can think about picking up coefficients of the wedge products $\Gamma_{\rho, i} \wedge \Gamma_{1, t}$ from $d \Gamma_{\rho, i}$ for $t=1,2$. For this purpose, one notices that in the Darboux-Cartan structure of $d \sigma_{\rho, i}$, visible in the structure equation $d \Gamma_{\rho, i}$, only wedge products of the form $\sigma_{\rho-1, j} \wedge \sigma_{1, t}$ can make nonzero coefficients of $\Gamma_{\rho, i} \wedge \Gamma_{1, t}$. In order to find these coefficients and according to Lemma 4.5 , if the coefficient of $d \sigma_{\rho, i}$ in the structure equation $d \Gamma_{\rho-1, j}$ is a (possibly zero) weight $\rho$ group parameter $a_{j_{r}}$, then the coefficient of $\Gamma_{\rho, i}$ in $\sigma_{\rho-1, j}$ is some fraction of the form $-\frac{a_{j_{r}}}{a_{1}^{\bar{a}} \bar{a}_{1}^{*}}$-notice that by considering the term $a_{j_{r}} d \sigma_{\rho, i}$ in the weight $-(\rho-1)$ structure equation $d \Gamma_{\rho-1, j}$, we find a weighted homogeneous equation of $\mathrm{S}$, expressing $a_{j_{r}}$ in terms of some lower weight group parameters. Then the coefficient of the sought wedge product $\Gamma_{\rho, i} \wedge \Gamma_{1, t}$ in $\sigma_{\rho-1, j} \wedge \sigma_{1, t}$ is the multiplication between the coefficient $-\frac{a_{j r}}{a_{1}^{a} \bar{a}_{1}^{*}}$ of $\Gamma_{\rho, i}$ in $\sigma_{\rho-1, j}$ and the coefficient of $\Gamma_{1, t}$ in $\sigma_{1, t}$, which is $\frac{1}{a_{1}}$ where $t=1$ and $\frac{1}{\bar{a}_{1}}$ where $t=2$. This implies that: (i) after absorption (25) and equating to zero the coefficients of $\Gamma_{\rho, i} \wedge \Gamma_{1,1}$ and $\Gamma_{\rho, i} \wedge \Gamma_{1,2}$ in the structure equation $d \Gamma_{\rho, i}$, one finds two equations:

$$
\sum_{j_{r}} \frac{a_{j_{r}}}{a_{1}^{\bullet} \bar{a}_{1}^{\bullet}}+p_{i} t_{1}+q_{i} \bar{t}_{2}=0 \text { and } \sum_{j_{r}^{\prime}} \frac{a_{j_{r}^{\prime}}}{a_{1}^{\bullet} \bar{a}_{1}^{\bullet}}+q_{i} \bar{t}_{1}+p_{i} t_{2}=0,
$$

where according to (33) they are actually two equations in terms of $a_{2}, a_{3}$ and some other weight $\rho$ group parameters $a_{j_{r}}$. (ii) In the system $\mathrm{S}$, one finds some polynomial equations which express $a_{j_{r}}$ s and $a_{j_{r}}^{\prime}$ s in terms of some lower weight group parameters.

Surprisingly, Proposition 5.4 and equations (33) imply that one can regard eventually the above two equations (35) in terms of only $a_{3}, a_{2}, a_{1}$ and their conjugations. Now to finalize constructing the desired subsystem, it remains only to add these already found equations to S.

Before attempt to solve the system S, let us summarize our practical method of its construction. It is divided into the following two parts which should be performed after the absorption step (25):

Part I For each structure equation:

$$
\begin{aligned}
d \Gamma_{\ell, m}= & \left(p_{m} \alpha+q_{m} \bar{\alpha}\right) \wedge \Gamma_{\ell, m}+\sum_{l \supsetneqq \ell} \delta_{i_{t}} \wedge \Gamma_{l, j}+\sum_{l \geq \ell+2} a_{j_{n}} d \sigma_{l, n} \\
& +\sum_{r} a_{j_{r}} d \sigma_{\ell+1, r}+a_{1}^{p_{m}} \bar{a}_{1}^{q_{m}} d \sigma_{\ell, m},
\end{aligned}
$$

with $\ell=1, \ldots, \rho-1$ and for each term $a_{j_{r_{0}}} d \sigma_{\ell+1, r_{0}}$ in its penultimate sum, equate to zero the coefficient of the wedge product $\Gamma_{\ell, i_{j}} \wedge \Gamma_{1, t_{r}}$, where $\sigma_{\ell, i_{j}} \wedge \sigma_{1, t_{r}}$ uniquely appears in

\footnotetext{
6 Actually in CR dimension 1, the reason of satisfying Beloshapka's maximum conjecture in the lengths $\rho \geqslant 3$ may refer to this part of our constructions. In fact, to provide two more weighted homogeneous equations for $a_{2}$ and $a_{3}$, we need some more structure equations than those of $d \Gamma_{2,3}, d \Gamma_{1,1}$ and $d \Gamma_{1,2}=\overline{d \Gamma_{1,1}}$. This means that we should at least have the next structure equation $d \Gamma_{3,4}$ which appears in the case of CR models which are of length $\rho \geqslant 3$.
} 
the Darboux-Cartan structure of $d \sigma_{\ell+1, r_{0}}$ according to Lemma 3.6. The achieved equation belongs to $\mathrm{S}$.

Part II For each weight $-\rho$ structure equation:

$$
d \Gamma_{\rho, i}=\left(p_{i} \alpha+q_{i} \bar{\alpha}\right) \wedge \Gamma_{\rho, i}+a_{1}^{p_{i}} \bar{a}_{1}^{q_{i}} d \sigma_{\rho, i},
$$

equate to zero all coefficients of $\Gamma_{\rho, i} \wedge \Gamma_{1, t}$ for $t=1,2$ and add the achieved equations to $\mathrm{S}$.

\subsection{Solving the picked up subsystem}

After constructing the weighted homogeneous polynomial system $\mathrm{S}$, now let us attempt to find the weighted projective variety $\mathbf{V}(\mathscr{I})$ of the polynomial ideal $\mathscr{I}=\langle\mathrm{S}\rangle$-namely the solution of the system $\mathrm{S}$-in the weighted projective space $\mathbb{P}(1,2,3, \ldots)$ (see, e.g., [11] for more details). Since the only weight 1 group parameter $a_{1}$ is assumed to be nonzero, then this variety does not contain any point at the infinity surface $a_{1}=0$. Assume that $\mathscr{I}^{\text {aff }} \subset \mathbb{C}\left[a_{2}, a_{3}, \ldots, a_{r}\right]$ is the affine ideal obtained as the dehomogenization of $\mathscr{I}$ by setting $a_{1}=1$. If $g$ is a weighted homogeneous polynomial in $\mathscr{I}$, then the following relation holds between it and its dehomogenization $g^{\text {deh }}$ (cf. [11, Theorem 5.16]):

$$
g\left(a_{1}, a_{2}, a_{3}, \ldots, a_{r}\right)=a_{1}^{\mathrm{w}-\mathrm{deg}} \cdot g^{\operatorname{deh}}\left(\frac{a_{2}}{a_{1}^{\left[a_{2}\right]}}, \frac{a_{3}}{a_{1}^{\left[a_{3}\right]}}, \ldots, \frac{a_{r}}{a_{1}^{\left[a_{r}\right]}}\right)
$$

where w-deg is the weight degree of the affine polynomial $g^{\text {deh }}$. By Proposition 5.4 we can still state that associated with each group parameter $a_{j}$ visible in $\mathscr{I}^{\text {aff }}$, there exists some (not necessarily weighted homogeneous, any more) polynomial in this ideal, expressed in terms of $a_{j}$ and some other group parameters (variables) of absolutely lower weights. Moreover, these polynomials are all linear [consider the equations of S after setting $a_{1}=1$ in (30), (33), (35)].

This indicates that after selecting some appropriate order $\prec$ on the extant group parameters $a_{\bullet}$ enjoying the property that $a_{i} \prec a_{j}$ whenever $\left[a_{i}\right]<\left[a_{j}\right]$, then the affine ideal $\mathscr{I}^{\text {aff }}$ is in fact in Noether normal position and according to the Finiteness Theorem [10, Theorem 6 and Corollary 7, pp. 230-1], the affine variety $\mathbf{V}\left(\mathscr{I}^{\text {aff }}\right)$ is zero dimensional containing just the origin $(0,0, \ldots, 0)$. Then according to the above equality (36), one concludes that the weighted projective variety $\mathbf{V}(\mathscr{I})$, or equivalently the solution set of the weighted homogeneous system $\mathrm{S}$, comprises some points of the concrete form:

$$
\mathbf{V}(\mathscr{I})=\left\{\left(a_{1}, 0,0, \ldots, 0\right), \quad a_{1} \neq 0\right\} .
$$

In other words, in the solution set of our weighted homogeneous system S, all the group parameters visible in it—but not necessarily all the group parameters appearing in our ambiguity matrix - take the value zero, identically. In particular, the two fundamental group parameters $a_{2}$ and $a_{3}$ shall be zero. But, thanks to Lemma 3.8, vanishing of these two group parameters is sufficient to assert that all the group parameters $a_{j}, j \neq 1$, appearing in the ambiguity matrix $\mathbf{g}$ should be normalized to zero;

Proposition 5.5 After sufficient steps of applying absorption and normalization on the structure equations of the equivalence problem to a totally nondegenerate CR model $M_{k}$ of $C R$ dimension 1 and codimension $k$, all the appearing group parameters $a_{j}$ with $j=2,3,4, \ldots$ vanish, identically. 
This immediately results in the reduction of our ambiguity matrix group $G$ (cf. (18)) to the simple diagonal matrix Lie group $G^{\text {red }}$ comprising matrices of the form:

$$
\mathbf{g}^{\text {red }}:=\left(\begin{array}{cccc}
a_{1}^{p} \bar{a}_{1}^{q} & 0 & \cdots & 0 \\
\vdots & \ddots & 0 & 0 \\
0 & \cdots & \bar{a}_{1} & 0 \\
0 & \cdots & 0 & a_{1}
\end{array}\right) .
$$

Concerning the Maurer-Cartan matrix $\omega_{\mathrm{MC}}$ visible in (21), all the Maurer-Cartan forms $\delta$ vanish identically and it reduces to a diagonal matrix with some combinations of the 1-forms $\alpha=\frac{d a_{1}}{a_{1}}$ and its conjugation at its diagonal. Finally, after vanishing of the group parameters $a_{2}, a_{3}, \ldots$, then all torsion coefficients $T_{j, m}^{i}$ vanish identically except those which were constant from the beginning of construction;

Proposition 5.6 After vanishing the group parameters $a_{2}, a_{3}, a_{4}, \ldots$, our structure equations convert into the simple constant type:

$$
d \Gamma_{\ell, i}:=\left(p_{i} \alpha+q_{i} \bar{\alpha}\right) \wedge \Gamma_{\ell, i}+\sum_{\substack{l+m=\ell \\ j, n}} c_{j, n}^{i} \Gamma_{l, j} \wedge \Gamma_{m, n} \quad(\ell=1, \ldots, \rho, \quad i=1, \ldots, 2+k)
$$

for some constant complex integers $\mathrm{c}_{j, n}^{i}$.

Proof According to (21), our structure equations were originally of the form:

$$
d \Gamma_{\ell, i}=\left(p_{i} \alpha+q_{i} \bar{\alpha}\right) \wedge \Gamma_{\ell, i}+\underbrace{}_{l \supsetneqq \ell} \delta_{i_{j}} \wedge \Gamma_{l, j}+\sum_{l \supsetneqq \ell} a_{i_{j}} d \sigma_{l, j}+a_{1}^{p_{i}} \bar{a}_{1}^{q_{i}} d \sigma_{\ell, i} .
$$

As mentioned, after vanishing of the group parameters $a_{2}, a_{3}, \ldots$ all the Maurer-Cartan forms $\delta_{\bullet}$ vanish identically and this kills the first sum $\sum_{l \supsetneqq \ell} \delta_{i_{j}} \wedge \Gamma_{l, j}$. For the second $\operatorname{sum} \sum_{l \supsetneqq \ell} a_{i j} d \sigma_{l, j}$ and according to Lemma 3.13, since all differentiations $d \sigma_{l, j}$ are of the weights $\Varangle-1$ (notice that here $l \supsetneqq \ell$ and $\ell \geqslant 1$ ) then all the group parameters $a_{i_{j}}$ are of the weights $\supsetneqq 1$ and hence none of them is $a_{1}$. This yields vanishing of this term, as well. Then, it suffices to consider the last term $a_{1}^{p_{i}} \bar{a}_{1}^{q_{i}} d \sigma_{\ell, i}$ of the above structure equation. According to the computed Darboux-Cartan structure in Proposition 3.5 we have:

$$
d \sigma_{\ell, i}:=\sum_{\beta+\gamma=\ell} c_{r, s} \sigma_{\beta, r} \wedge \sigma_{\gamma, s}
$$

On the other hand, our inverse matrix $\mathbf{g}^{-1}$ is now converted to the simple form:

$$
\left(\mathbf{g}^{\mathrm{red}}\right)^{-1}=\left(\begin{array}{cccc}
\frac{1}{a_{1}^{p} \bar{a}_{1}^{q}} & 0 & \cdots & 0 \\
\vdots & \ddots & 0 & 0 \\
0 & \cdots & \frac{1}{\bar{a}_{1}} & 0 \\
0 & \cdots & 0 & \frac{1}{a_{1}}
\end{array}\right)
$$

which through the equality $\Sigma=\left(\mathbf{g}^{\text {red }}\right)^{-1} \cdot \Gamma$, it results that:

$$
\sigma_{\beta, r} \wedge \sigma_{\gamma, s}=\frac{1}{a_{1}^{m_{r}} \bar{a}_{1}^{n_{s}}} \Gamma_{\beta, r} \wedge \Gamma_{\gamma, s}
$$


for some constant integers $m_{r}$ and $n_{s}$. Then, the last term $a_{1}^{p_{i}} \bar{a}_{1}^{q_{i}} d \sigma_{\ell, i}$ can be brought into a combination as:

$$
a_{1}^{p_{i}} \bar{a}_{1}^{q_{i}} d \sigma_{\ell, i}:=\sum_{\beta+\gamma=\ell} \mathrm{c}_{r, s} \frac{a_{1}^{p_{i}} \bar{a}_{1}^{q_{i}}}{a_{1}^{m_{r}} \bar{a}_{1}^{n_{s}}} \Gamma_{\beta, r} \wedge \Gamma_{\gamma, s} .
$$

On the other hand, these coefficients $c_{r, s} \frac{a_{1}^{p_{i}} \bar{a}_{1}^{q_{i}}}{a_{1}^{m_{r}} \bar{a}_{1}^{n_{s}}}$ are in fact the only remained torsion coefficients $T_{r s}^{i}$ of the wedge products $\Gamma_{\beta, r} \wedge \Gamma_{\gamma, s}$, in the structure equation $d \Gamma_{\ell, i}$ and hence according to Proposition 4.4, are of the weight zero. Since they involve just weight one group parameters $a_{1}$ and $\bar{a}_{1}$ then, after simplifications if necessary, they will be either some constants or some fractions of the form:

$$
\mathrm{C}_{r, s} \frac{a_{1}^{i}}{\bar{a}_{1}^{i}} \quad \text { or } \quad \mathrm{c}_{r, s} \frac{\bar{a}_{1}^{i}}{a_{1}^{i}} .
$$

Consequently, our structure equation $d \Gamma_{\ell, i}$ is now converted into the form:

$$
\begin{aligned}
d \Gamma_{\ell, i}= & \left(p_{i} \alpha+q_{i} \bar{\alpha}\right) \wedge \Gamma_{\ell, i}+\sum_{\beta^{\prime}+\gamma^{\prime}=\ell} c_{r^{\prime}, s^{\prime}} \Gamma_{\beta^{\prime}, r^{\prime}} \wedge \Gamma_{\gamma^{\prime}, s^{\prime}} \\
& +\sum_{\beta+\gamma=\ell} c_{r, s} \frac{a_{1}^{i}}{\bar{a}_{1}^{i}} \Gamma_{\beta, r} \wedge \Gamma_{\gamma, s}+\sum_{\beta+\gamma=\ell} c_{r, s} \frac{\bar{a}_{1}^{j}}{a_{1}^{j}} \Gamma_{\beta, r} \wedge \Gamma_{\gamma, s} .
\end{aligned}
$$

All the appearing $\beta$ s and $\gamma \mathrm{s}$ in this equation are absolutely less than $\ell$, whence in the case that one $c_{r, s}$ is nonzero then the torsion coefficient $T_{r, s}^{i}=\mathrm{c}_{r, s} \frac{a_{1}^{i}}{\bar{a}_{1}^{i}}$ or $T_{r, s}^{i}=\mathrm{c}_{r, s} \frac{\bar{a}_{1}^{i}}{a_{1}^{i}}$ of $\Gamma_{\beta, r} \wedge \Gamma_{\gamma, s}$ is essential and can be plainly normalized to some constant, say $c_{r, s}$, by normalizing $\frac{a_{1}}{\bar{a}_{1}}=1$, i.e., by considering the only remained parameter $a_{1}$ as real. Then all powers of $\frac{a_{1}}{\bar{a}_{1}}$ will be equal to 1 and consequently, we receive finally just some constant coefficients of these remaining wedge products.

\subsection{Normalization of $a_{1}$}

What mentioned at the end of the above proof demonstrates slightly normalization of the only remained group parameter $a_{1}$. Accordingly, this parameter is never normalizable when after vanishing the group parameters $a_{2}, a_{3}, \ldots$, all the torsion coefficients of the structure equations are constant. Otherwise, $a_{1}$ will be normalized just to a real group parameter.

Let us inspect more this normalization. For this purpose, we define the type of each initial vector field $\mathscr{L}_{\ell, i}$ as the pair $\left(p_{i}, q_{i}\right)$ where $p_{i}$ and $q_{i}$ are, respectively, the numbers of appearing $\mathscr{L}_{1,1}$ and $\mathscr{L}_{1,2}$ in the construction of $\mathscr{L}_{\ell, i}$ as an iterated bracket of them (cf. Lemma 3.7). After vanishing the group parameters $a_{2}, a_{3}, \ldots$, now we have the following relations between the initial and lifted forms:

$$
\Gamma_{\ell, i}=a_{1}^{p_{i}} \bar{a}_{1}^{q_{i}} \sigma_{\ell, i}
$$

where $\left(p_{i}, q_{i}\right)=\operatorname{type}\left(\mathscr{L}_{\ell, i}\right)$ is actually the type of $\mathscr{L}_{\ell, i}$. We have the following effective criterion concerning the normalization of $a_{1}$;

Proposition 5.7 There are only two possibilities for the normalization of the remained group parameter $a_{1}$ : it is either normalizable to a real group parameter or it is never normalizable. 
If the model $M_{k}$ is of the length $\rho \geqslant 4$, then the latter possibility occurs if and only if for each weight $-\rho$ initial 2-form $d \sigma_{\rho, i}$ with the Darboux-Cartan structure:

$$
d \sigma_{\rho, i}:=\sum_{\substack{r, s \\ \beta+\gamma=\rho}} \mathrm{c}_{r, s} \sigma_{\beta, r} \wedge \sigma_{\gamma, s}
$$

and for each wedge product $\sigma_{\beta, r} \wedge \sigma_{\gamma, s}$, visible in it, the equality:

$$
\text { type }\left(\mathscr{L}_{\rho, i}\right)=\text { type }\left(\mathscr{L}_{\beta, r}\right)+\text { type }\left(\mathscr{L}_{\gamma, s}\right)
$$

holds between the types of the corresponding initial fields.

Proof We shall prove only the second part of the assertion. As above, let us denote the type of each initial field $\mathscr{L}_{\ell, j}$ by $\left(p_{j}, q_{j}\right)$. Consider $\mathscr{L}_{1,1}, \ldots, \mathscr{L}_{\rho-1, t}$ as all initial vector fields of lengths $\supsetneqq \rho$. Whence $\mathscr{L}_{\rho, t+1}, \ldots, \mathscr{L}_{\rho, 2+k}$ are of the maximum length $\rho$. Also assume that $w_{\tilde{k}}$, with $\tilde{k} \leqslant k$, is the last complex coordinate of the weight $\rho-1$ in the defining equations (9) of $M_{k}$. Consequently, $\tilde{k}$ is the maximum possible number where $M_{\tilde{k}} \subset \mathbb{C}^{1+\tilde{k}}$, represented as the graph of the first $\tilde{k}$ defining equations of $M_{k}$, is of the length $\rho-1$. By the procedure of constructing initial frames and coframes introduced in Sect. 3, one observes for $i=1, \ldots, t$ that the associated Darboux-Cartan structure of $d \sigma_{\ell, i}$ is exactly of the same form:

$$
d \sigma_{\ell, i}:=\sum_{\substack{r, s \\ \beta+\gamma=\ell}} \mathrm{c}_{r, s} \sigma_{\beta, r} \wedge \sigma_{\gamma, s},
$$

in the both cases of $M_{k}$ and $M_{\tilde{k}}$. Furthermore, Proposition 3.11 says that the ambiguity matrix of $M_{\tilde{k}}$ actually stands inside that of $M_{k}$. Hence in the both cases of $M_{\tilde{k}}$ and $M_{k}$ and for each $i=1, \ldots, t$, the initial 2-form $d \sigma_{\ell, i}$ receives exactly the same linear combination [cf. (40)]:

$$
d \sigma_{\ell, i}:=\sum_{\substack{\beta+\gamma=\ell \\ r, s}} \frac{c_{r, s}}{a_{1}^{p_{r}+p_{s}} \bar{a}_{1}^{q_{r}+q_{s}}} \Gamma_{\beta, r} \wedge \Gamma_{\gamma, s},
$$

after vanishing the group parameters $a_{2}, a_{3}, \ldots$ (notice that $M_{\tilde{k}}$ is of the length $\rho-1 \geq$ 3 and thus in its structure equations, the group parameters $a_{2}, a_{3}, \ldots$ vanish as well as $M_{k}$ ). Regarding the final structure equations (39), thus the structure equations of $M_{\tilde{k}}$ are exactly of the same form as the first $t$ structure equations of $M_{k}$. This implies that $a_{1}$ can not be normalizable throughout these $t$ first structure equations since otherwise, the zeroth homogeneous component $\mathfrak{g}_{0}$ of $\mathfrak{a u t}_{C R}\left(M_{\tilde{k}}\right)$ is of dimension $<2$ (we will observe the reason in the next section). That is while according to the discussion presented at the bottom of the page 483 of [5], $\mathfrak{g}_{0}$ is of the maximum dimension two, in this specific case. Thus, the last group parameter $a_{1}$ can be normalizable only through the weight $-\rho$ structure equations. Considering:

$$
d \Gamma_{\rho, i}=\left(p_{i} \alpha+q_{i} \bar{\alpha}\right) \wedge \Gamma_{\ell, i}+a_{1}^{p_{i}} \bar{a}_{1}^{q_{i}} d \sigma_{\rho, i},
$$

as one of such equations with:

$$
d \sigma_{\rho, i}:=\sum_{\substack{r, s \\ \beta+\gamma=\rho}} \mathrm{c}_{r, s} \sigma_{\beta, r} \wedge \sigma_{\gamma, s}
$$


then, the last paragraphs of the proof of Proposition 5.6 show that $a_{1}$ is never normalizable if and only if in the expression [cf. (40)]:

$$
a_{1}^{p_{i}} \bar{a}_{1}^{q_{i}} d \sigma_{\rho, i}:=\sum_{\substack{\beta+\gamma=\rho \\ r, s}} c_{r, s} \frac{a_{1}^{p_{i}} \bar{a}_{1}^{q_{i}}}{a_{1}^{p_{r}+p_{s}} \bar{a}_{1}^{q_{r}+q_{s}}} \Gamma_{\beta, r} \wedge \Gamma_{\gamma, s},
$$

all the fractions $\frac{a_{1}^{p_{i}} q_{1}^{q_{i}}}{a_{1}^{p_{r}+p_{s}} \bar{a}_{1}^{q_{r}+q_{s}}}$ are reducible to a constant integer; hence if and only if:

$$
p_{i}=p_{r}+p_{s} \text { and } q_{i}=q_{r}+q_{s} .
$$

This completes the proof.

Remark 5.8 Taking into account the procedure of constructing Darboux-Cartan structures from the table of commutators of the initial frame of $M_{k}$ (cf. Lemma 3.4) and as a vector field version of the above criterion, one may state equivalently that $a_{1}$ is never normalizable if and only if when $\left[\mathscr{L}_{\beta, r}, \mathscr{L}_{\gamma, s}\right]$ with $\alpha+\beta=\rho$ includes a nonzero coefficient of a length $\rho$ vector field $\mathscr{L}_{\rho, i}$, then we have:

$$
\text { type }\left(\mathscr{L}_{\rho, i}\right)=\text { type }\left(\mathscr{L}_{\beta, r}\right)+\text { type }\left(\mathscr{L}_{\gamma, s}\right) \text {. }
$$

Example 5.9 In codimension $k=5$, a length four generic $\mathrm{CR}$ model $M_{5} \subset \mathbb{C}^{6}$ is represented as the graph of five defining polynomials:

$$
\begin{aligned}
& w_{1}-\bar{w}_{1}=2 i z \bar{z}, \\
& w_{2}-\bar{w}_{2}=2 i\left(z^{2} \bar{z}+z \bar{z}^{2}\right), \quad w_{3}-\bar{w}_{3}=2\left(z^{2} \bar{z}-z \bar{z}^{2}\right), \\
& w_{4}-\bar{w}_{4}=2 i\left(z^{3} \bar{z}+z \bar{z}^{3}\right)+2 i \mathbf{b} z^{2} \bar{z}^{2}, \quad w_{5}-\bar{w}_{5}=2\left(z^{3} \bar{z}-z \bar{z}^{3}\right)+2 i \mathbf{c} z^{2} \bar{z}^{2},
\end{aligned}
$$

for some two integers $\mathbf{b}, \mathbf{c} \in \mathbb{R}$. Performing required computations, one finds the expected initial 1-forms $\mathscr{L}_{1,1}, \mathscr{L}_{1,2}, \mathscr{L}_{2,3}, \mathscr{L}_{3,4}, \mathscr{L}_{3,5}, \mathscr{L}_{4,6}, \mathscr{L}_{4,7}$, with the associated types $(1,0)$, $(0,1),(1,1),(2,1),(1,2),(3,1),(1,3)$, respectively, and with the table of commutators:

\begin{tabular}{llllllll}
\hline & $\mathscr{L}_{1,1}$ & $\mathscr{L}_{1,2}$ & $\mathscr{L}_{2,3}$ & $\mathscr{L}_{3,4}$ & $\mathscr{L}_{3,5}$ & $\mathscr{L}_{4,6}$ & $\mathscr{L}_{4,7}$ \\
\hline $\mathscr{L}_{1,1}$ & 0 & $\mathscr{L}_{2,3}$ & $\mathscr{L}_{3,4}$ & $\mathscr{L}_{4,6}$ & $i \mathbf{r} \mathscr{L}_{4,6}+i \overline{\mathbf{r}} \mathscr{L}_{4,7}$ & 0 & 0 \\
$\mathscr{L}_{1,2}$ & $*$ & 0 & $\mathscr{L}_{3,5}$ & $-i \mathbf{r} \mathscr{L}_{4,6}-i \overline{\mathbf{r}} \mathscr{L}_{4,7}$ & $\mathscr{L}_{4,7}$ & 0 & 0 \\
$\mathscr{L}_{2,3}$ & $*$ & $*$ & 0 & 0 & 0 & 0 & 0 \\
$\mathscr{L}_{3,4}$ & $*$ & $*$ & $*$ & 0 & 0 & 0 & 0 \\
$\mathscr{L}_{3,5}$ & $*$ & $*$ & $*$ & $*$ & 0 & 0 & 0 \\
$\mathscr{L}_{4,6}$ & $*$ & $*$ & $*$ & $*$ & $*$ & 0 & 0 \\
$\mathscr{L}_{4,7}$ & $*$ & $*$ & $*$ & $*$ & $*$ & $*$ & 0 \\
\hline
\end{tabular}

for:

$$
\mathbf{r}:=\frac{1}{3}(\mathbf{b}-i \mathbf{c})
$$

Here, $\mathscr{L}_{4,6}$ and $\mathscr{L}_{4,7}$ are the only initial vector fields of the maximum length four. A glance on the above table shows that these two elements have some nonzero coefficients only in the Lie brackets:

$$
\begin{aligned}
& {\left[\mathscr{L}_{1,1}, \mathscr{L}_{3,4}\right]=\mathscr{L}_{4,6}, \quad\left[\mathscr{L}_{1,1}, \mathscr{L}_{3,5}\right]=i \mathbf{r} \mathscr{L}_{4,6}+i \overline{\mathbf{r}} \mathscr{L}_{4,7}} \\
& {\left[\mathscr{L}_{1,2}, \mathscr{L}_{3,5}\right]=\mathscr{L}_{4,7}, \quad\left[\mathscr{L}_{1,2}, \mathscr{L}_{3,4}\right]=-i \mathbf{r} \mathscr{L}_{4,6}-i \mathbf{r} \mathscr{L}_{4,7}}
\end{aligned}
$$


If $\mathbf{r}=0$, then the second and fourth brackets will be disregarded. It is easy to check concerning the two remained brackets $\left[\mathscr{L}_{1,1}, \mathscr{L}_{3,4}\right]=\mathscr{L}_{4,6}$ and $\left[\mathscr{L}_{1,2}, \mathscr{L}_{3,5}\right]=\mathscr{L}_{4,7}$ that we have:

type $\left(\mathscr{L}_{4,6}\right)=$ type $\left(\mathscr{L}_{1,1}\right)+$ type $\left(\mathscr{L}_{3,4}\right)$ and type $\left(\mathscr{L}_{4,7}\right)=\operatorname{type}\left(\mathscr{L}_{1,2}\right)+\operatorname{type}\left(\mathscr{L}_{3,5}\right)$.

This, according to Proposition 5.7, yields that the group parameter $a_{1}$ is never normalizable in this case. But, if $\mathbf{r} \neq 0$, then one finds a nonzero coefficient of $\mathscr{L}_{4,6}$ in the Lie bracket $\left[\mathscr{L}_{1,1}, \mathscr{L}_{3,5}\right]$. That is while the type of $\mathscr{L}_{4,6}$, namely $(3,1)$, is not equal to:

$$
\text { type }\left(\mathscr{L}_{1,1}\right)+\text { type }\left(\mathscr{L}_{3,5}\right)=(1,0)+(1,2)=(2,2) \text {. }
$$

This implies that in this case, the group parameter $a_{1}$ can be normalized to a real parameter. This result coincides with the case $K=5$ (ordinary type) of [35, Theorem 1].

\subsection{Prolongation}

At this stage that no further application of absorption-normalization is effective then, we have to decide whether it is possible proceeding into the third step of Cartan's method, namely the prolongation, or not. For this purpose, we shall check the so-called Cartan's arithmetic test of involutivity for the lifted coframe $\left\{\Gamma_{1,1}, \ldots, \Gamma_{\rho, 2+k}\right\}$ with the structure equations (38). We refer the reader to Chapter 11 of Olver's book [23] for necessary definitions and results concerning this subject (see also pp. 113-115 of the expanded version of [21] for a brief description).

Lemma 5.10 The Maurer-Cartan form $\alpha=\frac{d a_{1}}{a_{1}}$ is the only 1-form which can enjoy the system of structure equations (38).

Proof By Proposition 5.6, the structure equations of $d \Gamma_{2,3}$ and $d \Gamma_{1,1}$ are now of the form [see also (31)]:

$$
\begin{aligned}
& d \Gamma_{2,3}=(\alpha+\bar{\alpha}) \wedge \Gamma_{2,3}+\mathrm{c} \Gamma_{1,1} \wedge \Gamma_{1,2}, \\
& d \Gamma_{1,1}=\alpha \wedge \Gamma_{1,1},
\end{aligned}
$$

for some constant integer c. If $\alpha^{\prime}$ is another 1-form, enjoying the structure equations (38), then subtracting by pairs the above expressions of $d \Gamma_{2,3}$ and $d \Gamma_{1,1}$ with $\alpha$ and with $\alpha^{\prime}$, gives:

$$
\begin{aligned}
& 0 \equiv\left(\alpha+\bar{\alpha}-\alpha^{\prime}-\bar{\alpha}^{\prime}\right) \wedge \Gamma_{2,3}, \\
& 0 \equiv\left(\alpha-\alpha^{\prime}\right) \wedge \Gamma_{1,1} .
\end{aligned}
$$

Applying the well-known Cartan's Lemma [23, Exercise 1.33] on these equations implies that we have:

$$
\alpha-\alpha^{\prime}+\bar{\alpha}-\bar{\alpha}^{\prime}=A \Gamma_{2,3} \text { and } \alpha-\alpha^{\prime}=B \Gamma_{1,1}
$$

for some certain functions $A$ and $B$. If we substitute the second equality of (42) into the first one, then we plainly receive:

$$
B \Gamma_{1,1}+\bar{B} \overline{\Gamma_{1,1}}-A \Gamma_{2,3}=0 .
$$

Now, thanks to the equality $\overline{\Gamma_{1,1}}=\Gamma_{1,2}$ (cf. Sect. 3.1.1) and due to the fact that $\Gamma_{2,3}, \Gamma_{1,2}$ and $\Gamma_{1,1}$ are linearly independent, then we have $A=B=0$. Now, the second equation of (42) immediately implies that $\alpha^{\prime}=\alpha$ and hence the Maurer-Cartan form $\alpha$ is unique, as was claimed. 
This lemma shows that if we execute again the absorption procedure on the structure equation (38) by replacing $\alpha$ with:

$$
\alpha+r_{2+k} \Gamma_{\rho, 2+k}+\cdots+r_{1} \Gamma_{1,1},
$$

then in order to annihilate all the coefficients in the new expressions of $d \Gamma_{\bullet}, \bullet$, the only solution is $r_{2+k}=\cdots=r_{1}=0$. In other words, the number of free variables or equivalently the degree of indeterminancy is null ${ }^{7}$ and our final system of structure equations (38) is determinate. Thus, the lifted coframe $\left\{\Gamma_{1,1}, \ldots, \Gamma_{\rho, 2+k}\right\}$ is certainly non-involutive and we can start the prolongation step of Cartan's method.

In this determinate case, the main result behind the prolongation step is [23, Proposition 12.1]. It permits us to reformulate the current equivalence problem to our $(2+k)$-dimensional CR model $M_{k}$ on that of the bigger $(3+k)$ or $(4+k)$-dimensional prolonged space $M^{\mathrm{pr}}:=$ $M_{k} \times G^{\text {red }}$. For this, we have to add the remaining Maurer-Cartan forms $\alpha$ and $\bar{\alpha}$ to the original lifted coframe $\Gamma$ and consider $\left(\Gamma_{\rho, 2+k}, \ldots, \Gamma_{1,1}, \alpha, \bar{\alpha}\right)$ as the new lifted coframe associated with this prolonged space. In the case that $a_{1}$ is normalizable to a real group parameter, then of course we have $\alpha=\bar{\alpha}$. Constructing the associated structure equations to this new problem is easy, just adding $d \alpha=d\left(\frac{d a_{1}}{a_{1}}\right)=0$ to the former ones. Then, the final structure equations of our new equivalence problem to the prolonged space $M^{\mathrm{pr}}$ take the following $\{e\}$-structure constant type:

$$
\left[\begin{array}{l}
d \Gamma_{\ell, i}=\left(p_{i} \alpha+q_{i} \bar{\alpha}\right) \wedge \Gamma_{\ell, i}+\sum_{\ell_{1}+\ell_{2}=\ell} c_{j, n}^{i} \Gamma_{\ell_{1}, j} \wedge \Gamma_{\ell_{2}, n} \quad(\ell=1, \ldots, \rho, i=1, \ldots, 2+k), \\
d \alpha=0 \\
d \bar{\alpha}=0 .
\end{array}\right.
$$

Thus, we have arrived at the stage of stating the main result of this paper;

Theorem 5.1 The biholomorphic equivalence problem to a $(2+k)$-dimensional real analytic totally nondegenerate $C R$ model $M_{k} \subset \mathbb{C}^{1+k}$ of codimension $k$ is reducible to some absolute parallelisms, namely to some certain $\{e\}$-structures on prolonged manifolds $M_{k} \times G^{\text {red }}$ of real dimensions either $3+k$ or $4+k$.

Remark 5.11 The above final structure equations (44) together with Proposition 5.7 (or Remark 5.8) solve decisively the biholomorphic equivalence problem to Beloshapka's models of CR dimension one in terms of their associated initial frames (cf. [5, Corollary 7]). This result may have various applications in which let us state some of them as below:

(i) It supplies a practical tool to consider moduli spaces of model CR manifolds in CR dimension one (cf. [4,16] and in particular [29, Sect. 3]).

(ii) It also can provide one with the opportunity of constructing Cartan geometries of totally nondegenerate CR manifolds modeled on Beloshapka's models as is done in the specific codimensions $k=1,3$ in [22,33].

(iii) As is done in the short paper [28], the results enable us to provide an affirmative answer in CR dimension one to Beloshapka's question about the standardness of his models ( [5, Question 2]).

(iv) It also helps one to realize the structure of Lie algebras of infinitesimal CR automorphisms associated with the models. In the next section, we will discuss this idea in more details.

\footnotetext{
7 There is also another simpler way to show (by direct computations) that the degree of indeterminancy is zero in this case. It just requires to replace the only remaining Maurer-Cartan forms $\alpha$ and $\bar{\alpha}$ as (43) and observe that the result of applying absorption-normalization even only on the two structure equations (41) is nothing but $r_{2+k}=\cdots=r_{1}=0$. However, our reason of introducing Lemma 5.10 is that it may also have its own interest, independently.
} 
Weight assignment We assign naturally ${ }^{8}$ the weight zero to the new lifted 1 -forms $\alpha$ and $\bar{\alpha}$.

\section{Proof of Beloshapka's maximum conjecture in CR dimension one}

As we saw, the equivalence problem to a certain CR model $M_{k}$ converted finally to that of the prolonged space $M^{\mathrm{pr}}$ with the final constant type structure equations (44). According to [23, Theorem 8.16], if the final structure equations of an equivalence problem to an $r$-dimensional smooth manifold $M$ equipped with some lifted coframe $\left\{\gamma^{1}, \ldots, \gamma^{r}\right\}$ is of the constant type:

$$
d \gamma^{k}=\sum_{1 \leqslant i<j \leqslant r} c_{i j}^{k} \gamma^{i} \wedge \gamma^{j} \quad(k=1 \ldots r),
$$

then $M$ is (locally) diffeomorphic to an $r$-dimensional Lie group $\mathrm{G}$ corresponding to the Lie algebra $\mathfrak{g}$ with the basis elements $\left\{\mathrm{v}_{1}, \ldots, \mathrm{v}_{r}\right\}$ and enjoying the so-called structure constants:

$$
\left[\mathrm{v}_{i}, \mathrm{v}_{j}\right]=-\sum_{k=1}^{r} c_{i j}^{k} \mathrm{v}_{k} \quad(1 \leqslant i<j \leqslant r) .
$$

Let us try to find the Lie algebra $\mathfrak{g}$ corresponding to the constant structure equations (44). We associate to each lifted 1 -form $\Gamma_{\ell, i}$ of $M^{\text {pr }}$ the basis element $v_{\ell, i}$ of $\mathfrak{g}$. For the new appearing lifted 1 -forms $\alpha$ and $\bar{\alpha}$, let us associate $v_{0}$ and $v_{\overline{0}}$. If the real part of $a_{1}$ is normalizable (see Proposition 5.7), then we dispense with $\mathrm{v}_{\overline{0}}$ since in this case we have $\alpha=\bar{\alpha}$. Thus, our desired Lie algebra $\mathfrak{g}$ is of dimension either $3+k$ or $4+k$, depending on the normalization of $a_{1}$. Assign naturally the weight $-\ell$ to each basis element $v_{\ell, i}$ and the weight zero to $v_{0}$ and $v_{\overline{0}}$. In particular, because we do not see any wedge product $\alpha \wedge \bar{\alpha}$ among the structure equations (44) then, $\left[\mathrm{v}_{0}, \mathrm{v}_{\overline{0}}\right]=0$. This indicates that $\left\{\mathrm{v}_{0}, \mathrm{v}_{\overline{0}}\right\}$ generates an Abelian subalgebra of $\mathfrak{g}$.

Each structure equation $d \Gamma_{\ell, i}$ in (44) is some constant combination of the wedge products between lifted 1 -forms for which the sum of their weights is exactly $-\ell$. Thus, the Lie bracket between two weight $-\ell_{1}$ and $-\ell_{2}$ basis elements $v_{\bullet}$ of $\mathfrak{g}$ will be some constant combination of its weight $-\left(\ell_{1}+\ell_{2}\right)$ basis elements. Consequently, we have the following interesting result;

Proposition 6.1 Let $\mathfrak{g}_{-\ell}$ be the $\mathbb{C}$-vector space generated by all basis elements $\mathbf{v}_{\ell, i}$ of the weight $-\ell$ and let $\mathfrak{g}_{0}$ be the Abelian algebra generated by $\mathrm{v}_{0}$ and $\mathrm{v}_{\overline{0}}$. Then, the Lie algebra $\mathfrak{g}$ associated with the final structure equations (44) is graded of the form:

$$
\mathfrak{g}:=\underbrace{\mathfrak{g}_{-\rho} \oplus \mathfrak{g}_{-(\rho-1)} \oplus \cdots \oplus \mathfrak{g}_{-1}}_{\mathfrak{g}_{-}} \oplus \mathfrak{g}_{0}
$$

satisfying $\left[\mathfrak{g}_{-\ell_{1}}, \mathfrak{g}_{-\ell_{2}}\right]=\mathfrak{g}_{-\left(\ell_{1}+\ell_{2}\right)}$. In this case, $\mathfrak{g}_{-}$is $(2+k)$-dimensional and $\mathfrak{g}_{0}$ is of real dimension either 1 or 2 .

On the other hand, Corollary 14.20 of [23] says that this Lie algebra $\mathfrak{g}$ is in fact the symmetry Lie algebra of the prolonged space $M^{\mathrm{pr}}=M_{k} \times G^{\text {red }}$ with respect to its coframe $\left(\Gamma_{1,1}, \ldots, \Gamma_{\rho, 2+k}, \alpha, \bar{\alpha}\right)$; that is the Lie algebra associated with the Lie group $\mathrm{G}$ of selfequivalences $\Phi: M^{\mathrm{pr}} \rightarrow M^{\mathrm{pr}}$, satisfying $\Phi^{*}(\theta)=\theta$ for $\theta=\Gamma_{1,1}, \ldots, \Gamma_{\rho, 2+k}, \alpha, \bar{\alpha}$. But,

8 Notice that the exterior differentiation $d \alpha$ took the value zero, exactly as constant functions. 
according to [23, Proposition 12.1] and its proof, $\mathrm{G}$ can be identified with the CR symmetry Lie group $\operatorname{Aut}_{C R}(M)$ of biholomorphic maps $h: M_{k} \rightarrow M_{k}$, hence:

$$
\mathfrak{a u t}_{C R}\left(M_{k}\right) \cong \mathfrak{g} .
$$

Consequently similar to $\mathfrak{g}$, the Lie algebra $\mathfrak{a u t}_{C R}\left(M_{k}\right)$ will be graded without any positive component in its gradation, as was conjectured by Beloshapka.

Theorem 6.1 (Beloshapka's maximum conjecture in CR dimension one). The Lie algebra $\mathfrak{a u t}_{C R}\left(M_{k}\right)$ associated with a Beloshapka's real analytic totally nondegenerate $C R$ model $M_{k}$ of $C R$ dimension 1 , codimension $k$ and length $\rho \geq 3$-or equivalently of codimension $k \geq 2$-contains no any homogeneous component of absolutely positive homogeneity. In other words, such CR model has rigidity. Moreover, this Lie algebra is of dimension either $3+k$ or $4+k$.

Acknowledgements The author expresses his sincere thanks to Joël Merker and Amir Hashemi for their helpful comments, discussions and encouragements during the preparation of this paper. The research of the author was supported in part by a grant from IPM, No. 96510425.

\section{Appendix A: An illustrative example in length four}

By way of illustration the method introduced in Sect. 5, in this appendix we consider the biholomorphic equivalence problem to the 8-dimensional, length $\rho=4 \mathrm{CR}$ model $M_{6} \subset \mathbb{C}^{7}$ represented as the graph of six defining polynomials:

$$
\begin{aligned}
& w_{1}-\bar{w}_{1}=2 i z \bar{z}, \\
& w_{2}-\bar{w}_{2}=2 i\left(z^{2} \bar{z}+z \bar{z}^{2}\right), \quad w_{3}-\bar{w}_{3}=2\left(z^{2} \bar{z}-z \bar{z}^{2}\right), \\
& w_{4}-\bar{w}_{4}=2 i\left(z^{3} \bar{z}+z \bar{z}^{3}\right), \quad w_{5}-\bar{w}_{5}=2\left(z^{3} \bar{z}-z \bar{z}^{3}\right), \quad w_{6}-\bar{w}_{6}=2 i z^{2} \bar{z}^{2} .
\end{aligned}
$$

The assigned weights to the extant complex variables are:

$$
[z]=1, \quad\left[w_{1}\right]=2, \quad\left[w_{2}\right]=\left[w_{3}\right]=3, \quad\left[w_{4}\right]=\left[w_{5}\right]=\left[w_{6}\right]=4 .
$$

Saving the space, we do not present the intermediate calculations. According to our computations, our initial frame contains eight vector fields of various lengths $-1, \ldots,-4$ :

$$
\begin{aligned}
\mathscr{L} & :=\mathscr{L}_{1,1}, \quad \overline{\mathscr{L}}:=\mathscr{L}_{1,2}, \\
\mathscr{T} & :=\mathscr{L}_{2,3}=i[\mathscr{L}, \overline{\mathscr{L}}], \\
\mathscr{S} & :=\mathscr{L}_{3,4}=[\mathscr{L}, \mathscr{T}], \quad \overline{\mathscr{S}}:=\mathscr{L}_{3,5}=[\overline{\mathscr{L}}, \mathscr{T}], \\
\mathscr{U} & :=\mathscr{L}_{4,6}=[\mathscr{L}, \mathscr{S}], \quad \overline{\mathscr{U}}:=\mathscr{L}_{4,7}=[\overline{\mathscr{L}}, \mathscr{S}], \quad \mathscr{V}:=\mathscr{L}_{4,8}=[\mathscr{L}, \mathscr{S}]=[\overline{\mathscr{L}}, \mathscr{S}] .
\end{aligned}
$$

The other Lie brackets between these eight initial vector fields are all zero. Assume that:

$\Sigma:=(\underbrace{v_{0}, \mu_{0}, \bar{\mu}_{0}}_{\text {weight-4 }}, \underbrace{\sigma_{0}, \bar{\sigma}_{0}}_{\text {weight-3 }}, \underbrace{\rho_{0}}_{\text {weight-2 }}, \underbrace{\zeta_{0}, \bar{\zeta}_{0}}_{\text {weight-1 }})^{t}$ is the dual coframe of $(\mathscr{V}, \mathscr{U}, \overline{\mathscr{U}}, \mathscr{S}, \overline{\mathscr{S}}, \mathscr{T}, \mathscr{L}, \overline{\mathscr{L}})^{t}$.

Then the associated Darboux-Cartan structure to this coframe is:

$$
\begin{aligned}
& d \nu_{0}=\bar{\sigma}_{0} \wedge \zeta_{0}+\sigma_{0} \wedge \bar{\zeta}_{0}, \quad d \mu_{0}=\sigma_{0} \wedge \zeta_{0}, \quad d \bar{\mu}_{0}=\bar{\sigma}_{0} \wedge \bar{\zeta}_{0} \\
& d \sigma_{0}=\rho_{0} \wedge \zeta_{0}, \quad d \bar{\sigma}_{0}=\rho_{0} \wedge \bar{\zeta}_{0},
\end{aligned}
$$




$$
d \rho_{0}=i \zeta_{0} \wedge \bar{\zeta}_{0}, \quad d \zeta_{0}=0, \quad d \bar{\zeta}_{0}=0 .
$$

Assuming $\Gamma:=(\nu, \mu, \bar{\mu}, \sigma, \bar{\sigma}, \rho, \zeta, \bar{\zeta})^{t}$ as the associated lifted coframe, then our computation brings the ambiguity $8 \times 8$ invertible matrix of the biholomorphic equivalence problem to $M_{6}$ as:

$$
\Gamma=\underbrace{\left(\begin{array}{cccccccc}
a_{1}^{2} \bar{a}_{1}^{2} & 0 & 0 & 0 & 0 & 0 & 0 & 0 \\
0 & a_{1}^{3} \bar{a}_{1} & 0 & 0 & 0 & 0 & 0 & 0 \\
0 & 0 & a_{1} \bar{a}_{1}^{3} & 0 & 0 & 0 & 0 & 0 \\
a_{13} & a_{6} & 0 & a_{1}^{2} \bar{a}_{1} & 0 & 0 & 0 & 0 \\
\bar{a}_{13} & 0 & \bar{a}_{6} & 0 & a_{1} \bar{a}_{1}^{2} & 0 & 0 & 0 \\
a_{11} & a_{7} & \bar{a}_{7} & a_{3} & \bar{a}_{3} & a_{1} \bar{a}_{1} & 0 & 0 \\
a_{12} & a_{8} & \bar{a}_{9} & a_{4} & a_{5} & a_{2} & a_{1} & 0 \\
\bar{a}_{12} & a_{9} & \bar{a}_{8} & \bar{a}_{5} & \bar{a}_{4} & \bar{a}_{2} & 0 & \bar{a}_{1}
\end{array}\right)}_{\mathbf{g}}
$$

with the assigned weights:

$$
\left[a_{1}\right]=1, \quad\left[a_{2}\right]=2, \quad\left[a_{3}\right]=\left[a_{4}\right]=\left[a_{5}\right]=3, \quad\left[a_{6}\right]=\cdots=\left[a_{13}\right]=4 .
$$

By computing the (somehow big) inverse matrix $\mathbf{g}^{-1}$, one can check also the assertion of some results like Lemmas 4.2 and 4.3. Also, our Maurer-Cartan matrix is of the form:

$$
\omega_{\mathrm{MC}}:=\left(\begin{array}{cccccccc}
2 \alpha+2 \bar{\alpha} & 0 & 0 & 0 & 0 & 0 & 0 & 0 \\
0 & 3 \alpha+\bar{\alpha} & 0 & 0 & 0 & 0 & 0 & 0 \\
0 & 0 & \alpha+3 \bar{\alpha} & 0 & 0 & 0 & 0 & 0 \\
\delta_{13} & \delta_{6} & 0 & 2 \alpha+\bar{\alpha} & 0 & 0 & 0 & 0 \\
\bar{\delta}_{13} & 0 & \bar{\delta}_{6} & 0 & \alpha+2 \bar{\alpha} & 0 & 0 & 0 \\
\delta_{11} & \delta_{7} & \bar{\delta}_{7} & \delta_{3} & \bar{\delta}_{3} & \alpha+\bar{\alpha} & 0 & 0 \\
\delta_{12} & \delta_{8} & \bar{\delta}_{9} & \delta_{4} & \delta_{5} & \delta_{2} & \alpha & 0 \\
\bar{\delta}_{12} & \delta_{9} & \bar{\delta}_{8} & \bar{\delta}_{5} & \bar{\delta}_{4} & \bar{\delta}_{2} & 0 & \bar{\alpha}
\end{array}\right) \text {, with } \alpha=\frac{d a_{1}}{a_{1}} .
$$

Then, our structure equations will be of the form-we abbreviate the superfluous combinations of the wedge products $\delta_{j} \wedge \bullet$ just by some "..." since they will not play any important role:

$$
\begin{aligned}
& d \nu=(2 \alpha+2 \bar{\alpha}) \wedge \nu+a_{1}^{2} \bar{a}_{1}^{2} d \nu_{0}, \\
& d \mu=(3 \alpha+\bar{\alpha}) \wedge \mu+a_{1}^{3} \bar{a}_{1} d \mu_{0}, \\
& d \sigma=\cdots+(2 \alpha+\bar{\alpha}) \wedge \sigma+a_{13} d \nu_{0}+a_{6} d \mu_{0}+a_{1}^{2} \bar{a}_{1} d \sigma_{0}, \\
& d \rho=\cdots+(\alpha+\bar{\alpha}) \wedge \rho+a_{11} d \nu_{0}+a_{7} d \mu_{0}+\bar{a}_{7} d \bar{\mu}_{0}+a_{3} d \sigma_{0}+\bar{a}_{3} d \bar{\sigma}_{0}+a_{1} \bar{a}_{1} d \rho_{0}, \\
& d \zeta=\cdots+\alpha \wedge \zeta+a_{12} d \nu_{0}+a_{8} d \mu_{0}+\bar{a}_{9} d \bar{\mu}_{0}+a_{4} d \sigma_{0}+a_{5} d \bar{\sigma}_{0}+a_{2} d \rho_{0}+a_{1} d \zeta_{0} .
\end{aligned}
$$

Now, let us proceed as Sect. 5.2 to pick the appropriate weighted homogeneous system S. To do it and as is the method of absorption-normalization step, first we apply the substitutions:

$$
\begin{aligned}
\alpha & \mapsto \alpha+t_{8} v+t_{7} \mu+\cdots+t_{2} \bar{\zeta}+t_{1} \zeta, \\
\delta_{j} & \mapsto \delta_{j}+s_{8}^{j} v+s_{7}^{j} \mu+\cdots+s_{2}^{j} \bar{\zeta}+s_{1}^{j} \zeta, \quad(j=2, \ldots, 13)
\end{aligned}
$$

on the above structure equations. According to our proposed method of constructing S, in the minimum weight -4 structure equations $d \nu$ and $d \mu$, we have to compute the coefficients of $v \wedge\{\zeta, \bar{\zeta}\}$ and $\mu \wedge\{\zeta, \bar{\zeta}\}$, respectively. Moreover, in the weight -3 structure equation $d \sigma$, we should pick up the coefficients of $\sigma \wedge\{\zeta, \bar{\zeta}\}$ since $\sigma_{0} \wedge \zeta_{0}$ and $\sigma_{0} \wedge \bar{\zeta}_{0}$ uniquely appear 
in the Darboux-Cartan structure of the only extant length -4 differentiations $d \mu_{0}$ and $d \nu_{0}$ visible in this structure equation. Similarly, in the lengths -2 and -1 structure equations $d \rho$ and $d \zeta$, we should pick the coefficients of $\rho \wedge\{\zeta, \bar{\zeta}\}$ and $\zeta \wedge \bar{\zeta}$, respectively. Equating these coefficients to zero gives the following equations:

$$
\begin{aligned}
& \mathrm{S}_{d \nu}:=\left\{-\frac{\bar{a}_{13}}{a_{1}^{2} \bar{a}_{1}^{2}}=2 t_{1}+2 \bar{t}_{2}\right\}, \quad \mathrm{S}_{d \mu}:=\left\{-\frac{a_{6}}{a_{1}^{3} \bar{a}_{1}}=3 t_{1}+\bar{t}_{2}, \quad 0=\bar{t}_{1}+3 t_{2}\right\}, \\
& \mathrm{S}_{d \sigma}:=\left\{\frac{a_{6}}{a_{1}^{3} \bar{a}_{1}}-\frac{a_{3}}{a_{1}^{2} \bar{a}_{1}}=2 t_{1}+\bar{t}_{2}, \quad \frac{\bar{a}_{13}}{a_{1}^{2} \bar{a}_{1}^{2}}=\bar{t}_{1}+2 t_{2}\right\}, \\
& \mathrm{S}_{d \rho}:=\left\{\frac{a_{3}}{a_{1}^{2} \bar{a}_{1}}+i \frac{\bar{a}_{2}}{a_{1} \bar{a}_{1}}=t_{1}+\bar{t}_{2}\right\}, \quad \mathrm{S}_{d \zeta}:=\left\{i \frac{a_{2}}{a_{1} \bar{a}_{1}}=t_{2}\right\},
\end{aligned}
$$

where $S$ is the union of them. Putting the obtained expressions of the parameters $t_{1}$ and $t_{2}$ into these equations and multiplying them by sufficient powers of $a_{1}$ and $\bar{a}_{1}$, one finds the following weighted homogeneous system:

$$
\begin{aligned}
\mathrm{S}:= & \left\{\bar{a}_{13}+2 \bar{a}_{1} a_{3}+2 i a_{1} \bar{a}_{1} \bar{a}_{2}=0, \quad a_{6}+3 a_{1} a_{3}+5 i a_{1}^{2} \bar{a}_{2}=0, \quad \bar{a}_{3}+i \bar{a}_{1} a_{2}=0,\right. \\
& \left.a_{6}-3 a_{1} a_{3}-3 i a_{1}^{2} \bar{a}_{2}=0, \quad \bar{a}_{13}-a_{1} \bar{a}_{3}=0\right\} .
\end{aligned}
$$

Either by hand or by means of some computer softwares, one versifies that the solution of this system is nothing but $a_{2}=a_{3}=a_{6}=a_{13} \equiv 0$, which immediately implies vanishing of all the group parameters $a_{2}, a_{3}, a_{4}, \ldots, a_{13}$. Our computations shows that here $a_{1}$ is not normalizable. Applying these results and after one prolongation, the first structure equations (46) converts to the simple constant form:

$$
\begin{aligned}
& d \nu=(2 \alpha+2 \bar{\alpha}) \wedge v+\bar{\sigma} \wedge \zeta+\sigma \wedge \bar{\zeta}, \\
& d \mu=(3 \alpha+\bar{\alpha}) \wedge \mu+\sigma \wedge \zeta, \\
& d \sigma=(2 \alpha+\bar{\alpha}) \wedge \sigma+\rho \wedge \zeta, \\
& d \rho=(\alpha+\bar{\alpha}) \wedge \rho+i \zeta \wedge \bar{\zeta} \\
& d \zeta=\alpha \wedge \zeta \\
& d \alpha=0
\end{aligned}
$$

Proposition A.1 The Lie algebra $\mathfrak{g}$ associated with the above structure equations is 10dimensional with the basis $\left\{\mathrm{v}^{v}, \mathrm{v}^{\mu}, \mathrm{v}^{\bar{\mu}}, \mathrm{v}^{\sigma}, \mathrm{v}^{\bar{\sigma}}, \mathrm{v}^{\rho}, \mathrm{v}^{\zeta}, \mathrm{v}^{\bar{\zeta}}, \mathrm{v}^{\alpha}, \mathrm{v}^{\bar{\alpha}}\right\}$ and with the Lie brackets, displayed in the following table:

This Lie algebra, which is isomorphic to $\mathfrak{a u t}_{C R}\left(M_{6}\right)$, is graded of the form:

$$
\mathfrak{g}:=\mathfrak{g}_{-4} \oplus \mathfrak{g}_{-3} \oplus \mathfrak{g}_{-2} \oplus \mathfrak{g}_{-1} \oplus \mathfrak{g}_{0},
$$

with $\mathfrak{g}_{-4}=\left\langle\mathrm{v}^{v}, \mathrm{v}^{\mu}, \mathrm{v}^{\bar{\mu}}\right\rangle$, with $\mathfrak{g}_{-3}=\left\langle\mathrm{v}^{\sigma}, \mathrm{v}^{\bar{\sigma}}\right\rangle$, with $\mathfrak{g}_{-2}=\left\langle\mathrm{v}^{\rho}\right\rangle$, with $\mathfrak{g}_{-1}=\left\langle\mathrm{v}^{\zeta}, \mathrm{v}^{\bar{\zeta}}\right\rangle$ and with $\mathfrak{g}_{0}=\left\langle\mathrm{v}^{\alpha}, \mathrm{v}^{\alpha}\right\rangle$. 


\begin{tabular}{lllllllllll}
\hline & $\mathrm{v}^{v}$ & $\mathrm{v}^{\mu}$ & $\mathrm{v}^{\bar{\mu}}$ & $\mathrm{v}^{\sigma}$ & $\mathrm{v}^{\bar{\sigma}}$ & $\mathrm{v}^{\rho}$ & $\mathrm{v}^{\zeta}$ & $\mathrm{v}^{\bar{\zeta}}$ & $\mathrm{v}^{\alpha}$ & $\mathrm{v}^{\bar{\alpha}}$ \\
\hline $\mathrm{v}^{\nu}$ & 0 & 0 & 0 & 0 & 0 & 0 & 0 & 0 & $2 \mathrm{v}^{v}$ & $2 \mathrm{v}^{v}$ \\
$\mathrm{v}^{\mu}$ & $*$ & 0 & 0 & 0 & 0 & 0 & 0 & 0 & $3 \mathrm{v}^{\mu}$ & $\mathrm{v}^{\mu}$ \\
$\mathrm{v}^{\bar{\mu}}$ & $*$ & $*$ & 0 & 0 & 0 & 0 & 0 & 0 & $\mathrm{v}^{\bar{\mu}}$ & $3 \mathrm{v}^{\bar{\mu}}$ \\
$\mathrm{v}^{\sigma}$ & $*$ & $*$ & $*$ & 0 & 0 & 0 & $-\mathrm{v}^{\mu}$ & $-\mathrm{v}^{v}$ & $2 \mathrm{v}^{\sigma}$ & $\mathrm{v}^{\sigma}$ \\
$\mathrm{v}^{\bar{\sigma}}$ & $*$ & $*$ & $*$ & $*$ & 0 & 0 & $-\mathrm{v}^{v}$ & $-\mathrm{v}^{\bar{\mu}}$ & $\mathrm{v}^{\bar{\sigma}}$ & $2 \mathrm{v}^{\bar{\sigma}}$ \\
$\mathrm{v}^{\rho}$ & $*$ & $*$ & $*$ & $*$ & $*$ & 0 & $-\mathrm{v}^{\sigma}$ & $-\mathrm{v}^{\bar{\sigma}}$ & $\mathrm{v}^{\rho}$ & $\mathrm{v}^{\rho}$ \\
$\mathrm{v}^{\zeta}$ & $*$ & $*$ & $*$ & $*$ & $*$ & $*$ & 0 & $-i \mathrm{v}^{\rho}$ & $\mathrm{v}^{\zeta}$ & 0 \\
$\mathrm{v}^{\bar{\zeta}}$ & $*$ & $*$ & $*$ & $*$ & $*$ & $*$ & $*$ & 0 & 0 & $\mathrm{v}^{\bar{\zeta}}$ \\
$\mathrm{v}^{\alpha}$ & $*$ & $*$ & $*$ & $*$ & $*$ & $*$ & $*$ & $*$ & 0 & 0 \\
$\mathrm{v}^{\bar{\alpha}}$ & $*$ & $*$ & $*$ & $*$ & $*$ & $*$ & $*$ & $*$ & $*$ & 0
\end{tabular}

\section{References}

1. Baouendi, M.S., Ebenfelt, P., Rothschild, L.P.: Real Submanifolds in Complex Space and Their Mappings. Princeton University Press, Princeton (1999)

2. Beloshapka, V.K.: Model-surface method: an infinite-dimensional version. Proc. Steklov Inst. Math. 279, 14-24 (2012)

3. Beloshapka, V.K.: A generic CR-manifold as an $\{e\}$-structure. Russ. J. Math. Phys. 14(1), 1-7 (2007)

4. Beloshapka, V.K.: Moduli spaces of model real submanifolds. Russ. J. Math. Phys. 13(3), 245-252 (2006)

5. Beloshapka, V.K.: Universal models for real submanifolds. Math. Notes 75(4), 475-488 (2004)

6. Beloshapka, V.K.: Polynomial models of real manifolds. Izv. Math. 65(4), 641-657 (2001)

7. Beloshapka, V.K., Kossovskiy, I.: Classification of homogeneous CR-manifolds in dimension 4. Math. Anal. Appl. 374, 655-672 (2011)

8. Boggess, A.: CR Manifolds and the Tangential Cauchy-Riemann Complex. Studies in Advanced Mathematics. CRC Press, Boca Raton (1991)

9. Chern, S.S., Moser, Y.: Real hypersurfaces in complex manifold. Acta Math. 133, 219-271 (1974)

10. Cox, D.A., Little, L., O'Shea, D.: Ideals, Varieties and Algorithms: An Introduction to Computational Algebraic Geometry and Commutative Algebra, 2nd edn. Springer, Berlin (1997)

11. Decker, W., Lossen, C.: Computing in Algebraic Geometry: A Quick Start Using Singular, Algorithms and Computation in Mathematics, vol. 16. Springer, Berlin (2006)

12. Gammel, R.V., Kossovskiy, I.: The envelope of holomorphy of a model surface of the third degree and the 'rigidity' phenomenon. Proc. Steklov Inst. Math. 253(2), 22-37 (2006)

13. Isaev, A., Zaitsev, D.: Reduction of five-dimensional uniformaly Levi degenerate CR structures to absolute parallelisms. J. Geom. Anal. 23(3), 1571-1605 (2013)

14. Kossovskiy, I.: On envelopes of holomorphy of model manifolds. Izv. Math 71(3), 545-571 (2007)

15. Krantz, S.G.: Function Theory of Several Complex Variables, 2nd edn. AMS Chelsea Publishing, New York (2001)

16. Mamai, I.B.: Moduli spaces of model surfaces with one-dimensional complex tangent. Izv. Math. 77(2), 354-377 (2013)

17. Merker, J.: Equivalences of 5-dimensional CR manifolds, IIII: six models and (very) elementary normalizations. arXiv: 1311.7522

18. Merker, J., Lie, S.: Friedrich Engel's Theory of Transformation Groups (Vol. I, 1888). Modern Presentation and English Translation. Springer, Berlin (2015)

19. Merker, J., Pocchiola, S., Sabzevari, M.: Equivalences of 5-dimensional CR manifolds, II: general classes $I, I I, I I I_{1}, I I I_{2}, I V_{1}, I V_{2}, 5$ figures. arXiv: 1311.5669

20. Merker, J., Porten, E.: Holomorphic extension of CR functions, envelopes of holomorphy and removable singularities. Int. Math. Res. Surv. 2006, Article ID 28295

21. Merker, J., Sabzevari, M.: Cartan equivalence problem for 5-dimensional bracket-generating CRmanifolds in $\mathbb{C}^{4}$. J. Geom. Anal. 26(4), 3194-3251 (2016). Expanded form arXiv:1401.4297v1

22. Merker, J., Sabzevari, M.: Explicit expression of Cartan's connections for Levi-nondegenerate 3-manifolds in complex surfaces, and identification of the Heisenberg sphere. Cent. Eur. J. Math. 10(5), 1801-1835 (2012)

23. Olver, P.J.: Equivalence, Invariants and Symmetry. Cambridge University Press, Cambridge (1995)

24. Pocchiola, S.: Le Problème d'èquivalence Pour les Variétés de Cauchy-Riemann en Dimension 5, Ph.D. thesis, Paris-Sud 11 (2014) 
25. Poincaré, H.: Les fonction analytiques de deux variables et la représentation conforme. Rend. Circ. Math. Palermo 23, 185-220 (1907)

26. Reutenauer, C.: Free Lie Algebras, London Mathematical Society Monographs, New Series 7, Oxford University Press (1993)

27. Sabzevari, M.: On the maximum conjecture. Forum Math. 30(6), 1599-1608 (2018)

28. Sabzevari, M.: Totally nondegenerate models and standard manifolds in CR dimension one (submitted)

29. Sabzevari, M.: Moduli spaces of model real submanifolds: two alternative approaches. Sci. China Math. 58(11), 2261-2278 (2015)

30. Sabzevari, M., Hashemi, A., Alizadeh, B.M., Merker, J.: Applications of differential algebra for computing Lie algebras of infinitesimal CR-automorphisms. Sci. China Math. 57(9), 1811-1834 (2014)

31. Sabzevari, M., Hashemi, A., Alizadeh, B.M., Merker, J.: Lie algebras of infinitesimal CR-automorphisms of weighted homogeneous and homogeneous CR-generic submanifolds of $\mathbb{C}^{N}$. FiloMat 30(6), 1387-1411 (2016)

32. Sabzevari, M., Merker, J.: The Cartan equivalence problem for Levi-non-degenerate real hypersurfaces $M^{3} \subset \mathbb{C}^{2}$. Izv. Math. 78(6), 1158-1194 (2014)

33. Sabzevari, M., Merker, J., Pocchiola, S.: Canonical Cartan connections on maximally minimal generic submanifolds $M^{5} \subset \mathbb{C}^{4}$. Elect. Res. Ann. Math. Sci. (ERA-MS) 21, 153-166 (2014)

34. Shananina, E.N.: Polynomial models of degree 5 and algebras of their automorphisms. Math. Notes 75(5), 702-716 (2004)

35. Shananina, E.N.: Models for CR-manifolds of type $(1, K)$ for $3 \leq K \leq 7$ and their automorphisms. Math. Notes 67(3), 382-388 (2000) 